\title{
On the Mechanism of Niobium Electropolishing
}

\section{THESIS}

Presented in Partial Fulfillment of the Requirements for the Degree Master of Science in the Graduate School of The Ohio State University

\author{
By \\ Ashwini Chandra \\ Graduate Program in Materials Science and Engineering \\ The Ohio State University \\ 2012 \\ Master's Examination Committee: \\ Dr Gerald Frankel, Advisor \\ Dr Michael Sumption, Co-Advisor \\ Dr Rudolph Buchheit
}




\section{Copyright by}

Ashwini Chandra

2012 


\begin{abstract}
Pure Niobium is the material used to make Superconducting Radio Frequency (SRF) cavities. These cavities need to be free from surface defects like pits, bumps and protrusions to achieve the best performance. Electropolishing is commonly used as a final process to give a good surface finish to the niobium surface. The standard electrolyte used for electropolishing niobium is a 1:9 ratio of $\mathrm{HF}(48 \%)$ and $\mathrm{H}_{2} \mathrm{SO}_{4}(96 \%)$ respectively.

The mechanism of electropolishing is not fully understood and also the best electropolishing parameters need to be determined. Acid concentration, stirring, temperature and amount of material removed are the parameters expected to affect the surface finish. Their effect is studied using electrochemical polarization measurement, galvanostatic and potentiostatic techniques. The electro-dissolution occurring needs to be under mass transport control for the phenomenon of electropolishing to occur. This is characterized by the presence of a current density plateau in the niobium polarization curves. However Galvanostatic experimental result analysis points to other interfering transport processes as well. Results based on microscopy, surface profile measurement and current transient measurements for different electrode configurations indicate the presence of natural convection as one of the reason which could be causing deviation from a perfect semi infinite linear diffusion model.
\end{abstract}




\section{Dedication}

This document is dedicated to my family. 


\section{Acknowledgment}

First and foremost, I would like to thank my advisor, Dr. Gerald Frankel for his highly insightful and timely suggestions throughout the course of my stay at the Ohio State University as a graduate student. I also want to extend my sincerest thanks to my coadvisor, Dr. Michael Sumption for his valuable inputs on my research project. I would also like to thank Dr. Rudolph Buchheit and Dr. Edward Collings for their thoughts on my research efforts.

I would like to express my sincere appreciation to Dr. Saikat Adhikari, Dr. Ralf Posner, Dr. Mariano Kappes and Ms. Brendy Rincon Troconis for helping me set up my experiments. I consider myself really fortunate to be part of two lively and diverse research groups - FCC and CSMM. My special thanks to Xiaoji Li, Severine Cambier, Omar Lopez Garrity, Pitichon Klomjit, Cory Myers, Jinwook Seong, Sean Morton, Meng Tong, John Guzowski and Mike Susner for making my stay enjoyable at the lab. I am indebted to Ms Christine Putnam for her timely help in purchasing lab supplies, Dr Milan Majoros for help with programming LabVIEW and Dr. Dominique Verrault for helping me with English translation of a German paper. 
I am thankful to the technical staffs in the MSE department and the Physics Machine shop staffs at the Ohio State University for helping me prepare samples and build up my experimental set up.

Lastly, and most importantly, I would like to express my deepest gratitude to my parents for their love, constant support and firm faith in me. 


\section{Vita}

Indian Institute of Technology Roorkee
India
Department of Materials Science \&
Engineering, The Ohio State University,
USA.

\section{Fields of Study}

Major Field: Materials Science and Engineering 


\section{Table of Contents}

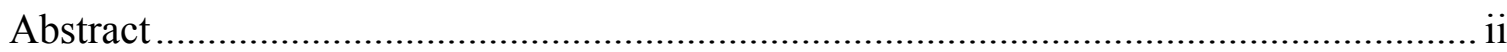

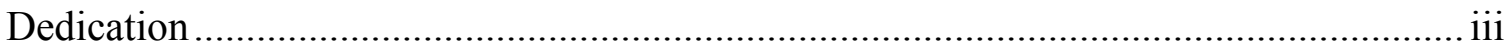

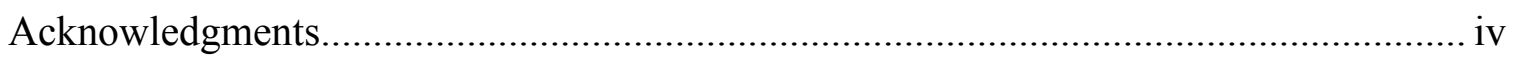

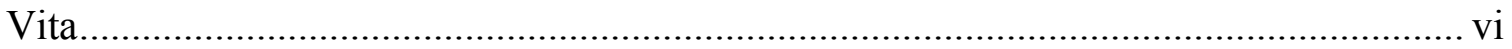

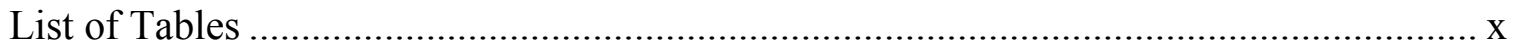

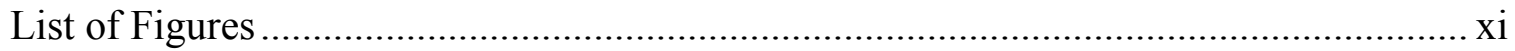

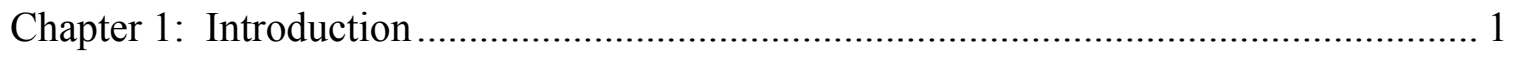

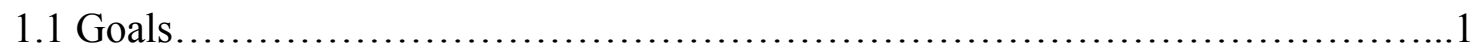

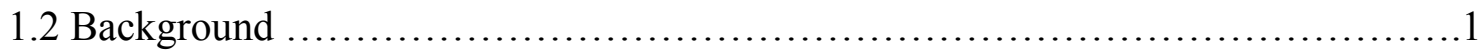

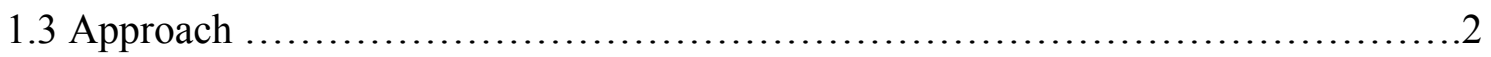

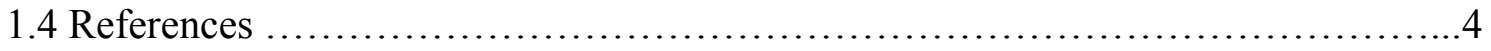

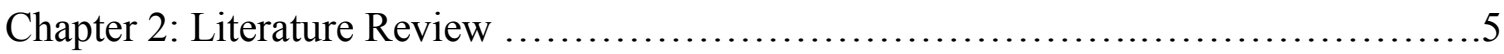

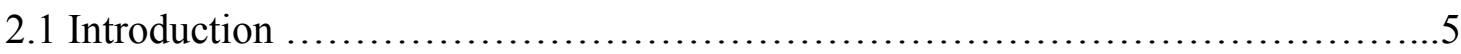

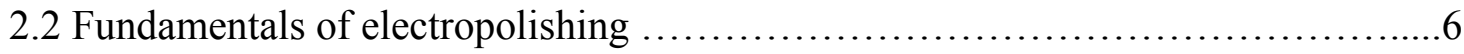

2.3 Different regions of electropolishing polarization curve $\ldots \ldots \ldots \ldots \ldots \ldots \ldots \ldots \ldots$

2.4 Electropolishing mechanisms .............................................11 


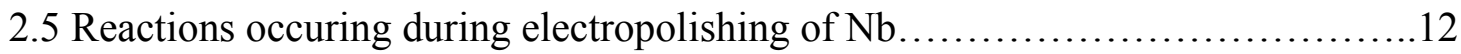

2.6 Optimum voltage consideration for electropolishing .........................13

2.7 Surface phenomena occuring during electropolishing...................... 14

2.8 Phenomenon of electropolishing of pit bottoms in pittin corrosion.............. 18

2.9 Phenomenon of pitting in anodic dissolution of metals and alloys.............. 20

2.9.1 Role of oxygen evolution in defect formation.......................... 21

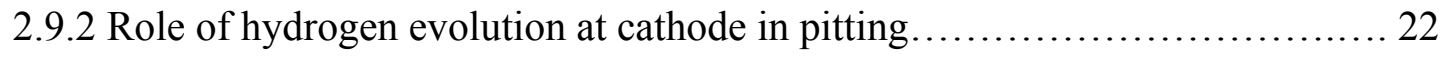

2.9.3 Other factors resulting in pitting during electropolishing...................23

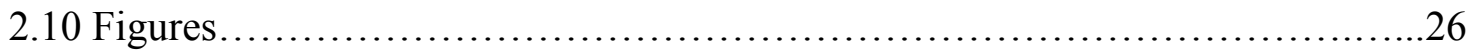

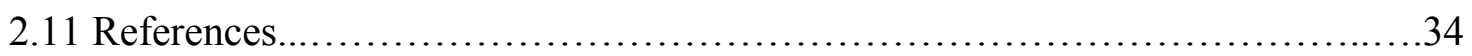

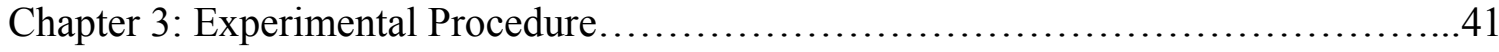

3.1 Material and sample preparation..................................... 41

3.2 Electrolyte preparation..............................................42

3.3 Experimental setup.................................................42

3.4 Electrochemical polarization experiments...............................44

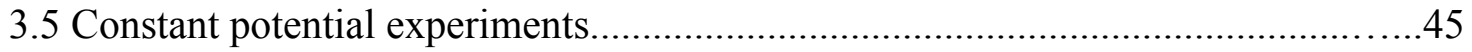

3.6 Galvanostatic experiments......................................... 45

3.7 Surface characterization.............................................46

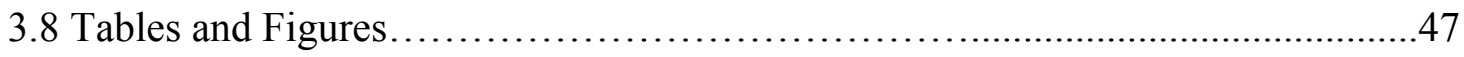

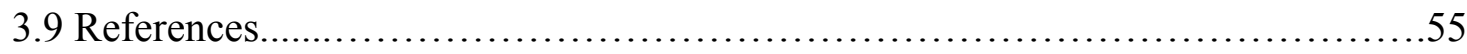

Chapter 4: Results and Discussion...........................................56

4.1 General shape of $\mathrm{Nb}$ electrochemical polarization curve.....................56 
4.2 Determination of important parameters affecting the electropolishing process of

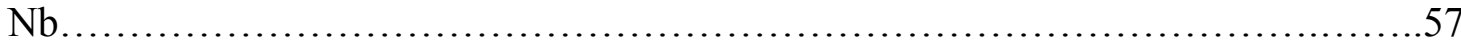

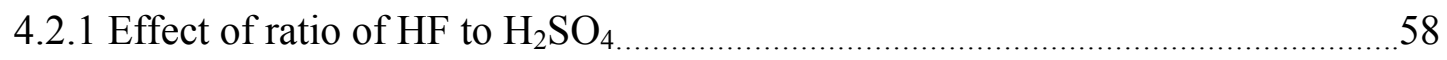

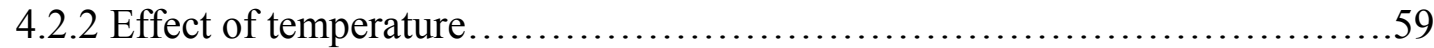

4.2.3 Effect of convection.............................................60

4.2.4 Effect of bath age.....................................................

4.2.5 Effect of cell voltage..............................................62

4.2.6 Effect of duration of electropolishing ................................62

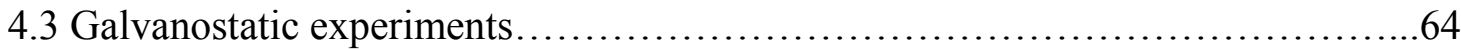

4.4 Role of hydrodynamics in electropolishing of niobium $\ldots \ldots \ldots \ldots \ldots \ldots \ldots \ldots . . .72$

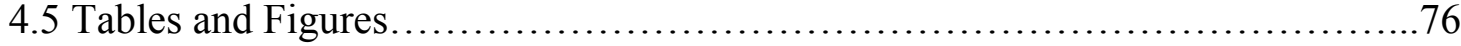

4.6 References....................................................... 103

Chapter 5: Conslusions................................................... 105

Chapter 6: Future Work.................................................. 108

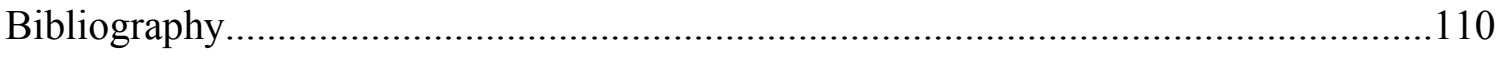




\section{List of Tables}

Table 3.1. Fluoride ion and sulfate ion concentrations corresponding to different volume

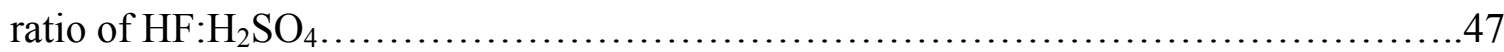

Table 4.1. Effect of Polishing voltage on RMS roughness for 1 hour electropolishing at

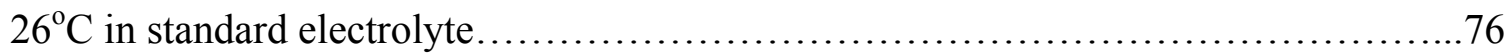

Table 4.2. Effect of electropolishing duration on RMS roughness and niobium thickness

removed for electropolishing at a cell voltage of $15 \mathrm{~V}$ at $26^{\circ} \mathrm{C}$ in standard electrolyte....76 


\section{List of Figures}

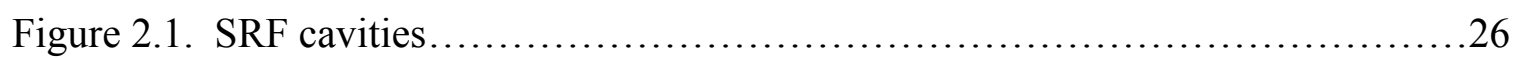

Figure 2.2. Electropolishing set up at Fermilab showing an assembled niobium single cell

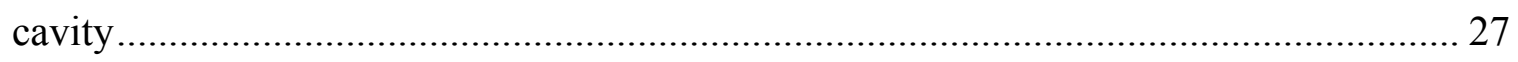

Figure 2.3. Concentration gradient difference over protrusions and valleys .................... 28

Figure 2.4. A typical polarization curve for electropolishing systems..................29

Figure 2.5. Concentration profile of various species for the three electropolishing

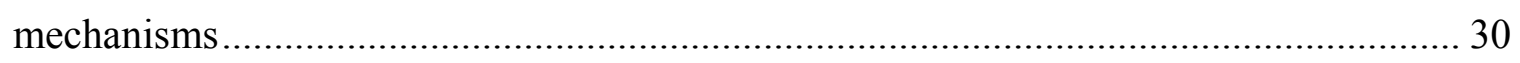

Figure 2.6. i vs. t curve measured for $\mathrm{Ti}$ in $3 \mathrm{M}$ methanol-sulfuric acid electrolyte ........ 31

Figure 2.7. Duplex salt film model schematic representation .......................................... 32

Figure 2.8. Schematic representation of adsorbate acceptor model in EP ........................ 33

Figure 3.1. Niobium electrode in flag configuration................................48

Figure 3.2. Masked niobium electrode with central circular area exposed...............49

Figure 3.3. Teflon sample holder for working electrode with an exposed circular central

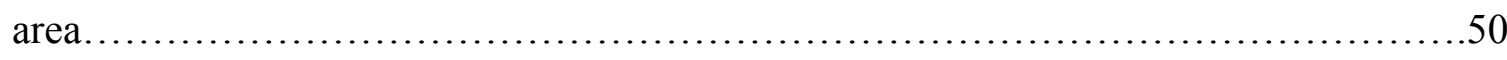

Figure 3.4. Schematic of the experimental set up for the tests........................51

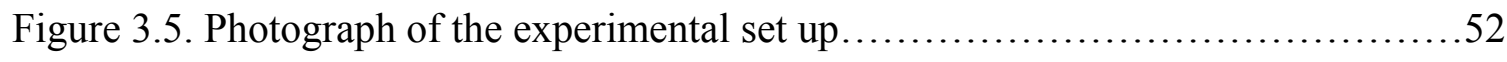


Figure 3.6. Photograph of the electronic equipments and the data acquisition

Figure 3.7. Veeco's contour GT K1 optical profilometer............................54

Figure 4.1. General $\mathrm{Nb}$ electrochemical polarization curve.........................77

Figure 4.2. Effect of acid ratio on $\mathrm{Nb}$ polarization curve at a temperature of $26^{\circ} \mathrm{C} \ldots \ldots . .78$

Figure 4.3. Linear variation of limiting current density with bulk fluoride ion

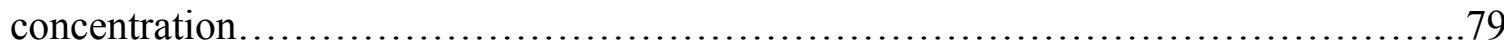

Figure 4.4. Effect of temperature on the $\mathrm{Nb}$ polarization curve for standard electrolytic

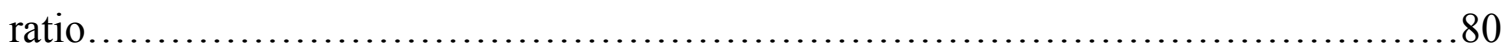

Figure 4.5 . Limiting current density variation with temperature...................... 81

Figure 4.6. Effect of stirring on $\mathrm{Nb}$ polarization curve............................ 82

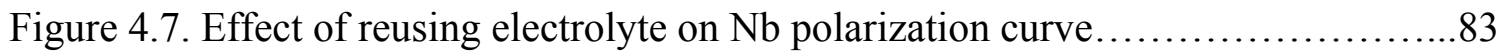

Figure 4.8a. Surface topography of $\mathrm{Nb}$ electropolished at an applied cell voltage of $5 \mathrm{~V}$

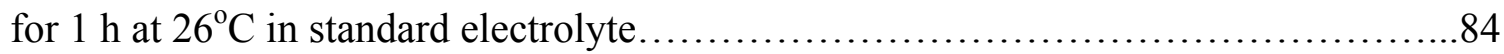

Figure 4.8b. Surface topography of $\mathrm{Nb}$ electropolished at an applied cell voltage of $10 \mathrm{~V}$

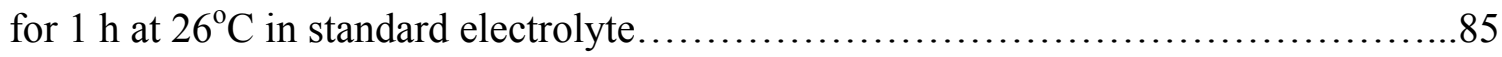

Figure 4.8c. Surface topography of $\mathrm{Nb}$ electropolished at an applied cell voltage of $15 \mathrm{~V}$

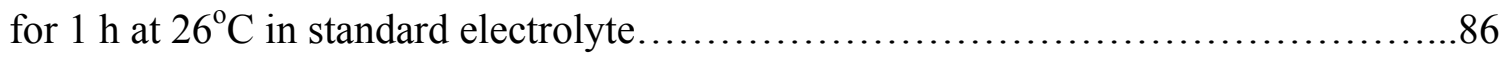

Figure 4.8d. Surface topography of $\mathrm{Nb}$ electropolished at an applied cell voltage of $20 \mathrm{~V}$

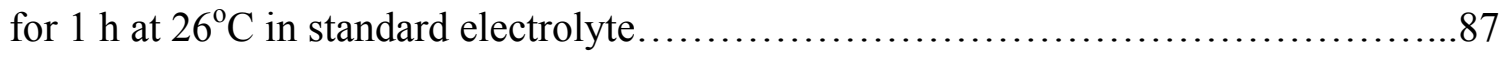

Figure 4.9a. Surface topography of niobium before electropolishing................. 88

Figure 4.9b. 3D Surface topography of $\mathrm{Nb}$ electropolished at an applied cell voltage of 15

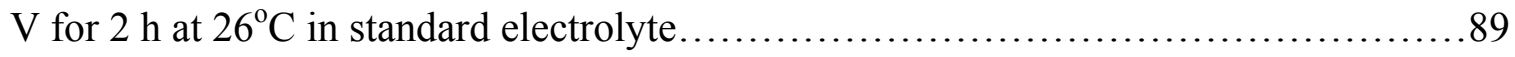


Figure 4.9c. 3D Surface topography of $\mathrm{Nb}$ electropolished at an applied cell voltage of 15

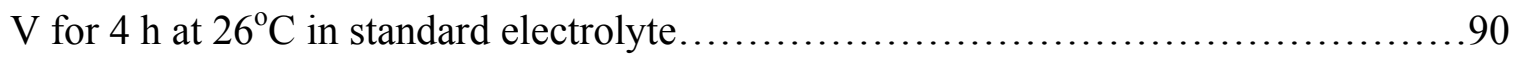

Figure 4.9d. 3D Surface topography of $\mathrm{Nb}$ electropolished at an applied cell voltage of 15 $\mathrm{V}$ for $6 \mathrm{~h}$ at $26^{\circ} \mathrm{C}$ in standard electrolyte...... .91

Figure 4.10. Potential transient for an applied current density on or above the limiting current density 92

Figure 4.11. Potential transient for an applied current density below the limiting current density. .93

Figure 4.12. Simulated variation of concentration polarization with time. .94

Figure 4.13. Potential transients demonstrating the increase in transient time with decreasing current density. .95

Figure 4.14. Effect of current density on transition time for constant acid ratio .96 Figure 4.15. Effect of fluoride ion concentration on transition time for a constant applied current density. .97

Figure 4.16a. Masked $\mathrm{Nb}$ sample electropolished in the exposed circular area..........98

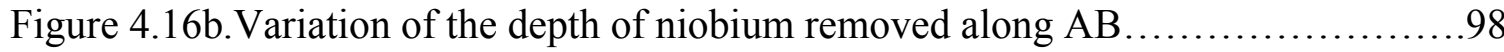
Figure 4.17. Ridges observed at the bottom edge of flag electrode sample.

Figure 4.18. Schematic depicting natural convection resulting from denser electrolyte near the working electrode surface because of salt film formation 100 Figure 4.19. Pits at the bottom surface of an electropolished $\mathrm{Nb}$ flag electrode sample.101 Figure 4.20. Current transients emphasizing the effect of sample configuration in the electrolyte. 


\section{Chapter 1 Introduction}

\subsection{Goals}

The goal of this project is to investigate the important parameters affecting the electropolishing of niobium. The mechanism involved in the process was also investigated using electrochemical tests. Surface finish dependence on duration of electropolishing and cell voltages was also studied.

\subsection{Background}

High purity niobium is used in making Superconducting Radio Frequency (SRF) cavities which find application in charged particle accelerators. The high energy beams generated by these accelerators is used in elementary particle research, nuclear physics research and free electron lasers [1]. These SRF cavities are manufactured by forming niobium sheets either by deep drawing or spinning [1]. After the forming operation, they are trimmed on a lathe or milling machine to attain a cup shape [1]. They are then electron beam welded in a vacuum chamber at the iris and equator region to generate the final shape [1]. Surface preparation is the next step in which the mechanically damaged layer due to sheet rolling and shaping operation (about100 - 200 $\mu \mathrm{m}$ ) is removed [2]. It has been found that surface defects like pits, bumps and protrusions are detrimental to the performance of the SRF cavities [3]. Buffered chemical polishing (BCP) which involves chemical etching 
with a $1: 1: 1$ or $1: 1: 2$ mixture of $\mathrm{HF}$ to $\mathrm{HNO}_{3}$ to $\mathrm{H}_{3} \mathrm{PO}_{4}$ is used as one of the steps in cavity processing at some laboratories $[4,5]$. However it limited the performance of the cavities due to the steps which the process created at the grain boundaries [2]. Electropolishing SRF cavities using a 1:9 volume ratio of $\mathrm{HF}$ to $\mathrm{H}_{2} \mathrm{SO}_{4}$ helped achieve higher accelerating gradients by not only removing the damaged layer but also providing a superior surface finish than BCP [1]. There are problems associated with electropolishing niobium as well mainly in the form of pitting close to the electron beam welded region. The first step in solving the problem is to develop a better understanding of the mechanism of niobium electropolishing. Qualitative studies of the process has been undertaken by various authors; however a quantitative determination has been lacking [6, 7]. The present study aims to quantitatively address the problem and also highlight other important processes which could be occurring. This would help in better controlling the industrial niobium electropolishing process to produce better surface finish which would further result in higher accelerating gradients.

\subsection{Approach}

Electrochemical polarization tests were carried out to determine the important parameters affecting the electropolishing process. Surface profile measurements using optical profilometry were done to ascertain the surface finish under different experimental conditions. Galvanostatic experiments were conducted to obtain potential transients. Useful information pertaining to the electropolishing mechanism involved was obtained by analyzing the data. Correlating the data to Sand's equation not only helped confirm 
the mechanism but also gave indications of presence of other interfering processes. Interpretation of results based on microscopic observations, surface profile measurements and current transient measurement for different electrode configuration pointed to the presence of natural convection in the niobium electropolishing system. 


\subsection{References}

1. H.Padamsee, J.Knobloch, and T.Hays, $R F$ Superconductivity for Accelerators. 2 ed. 2008, Morlenbach: Wiley.

2. H.Padamsee, RF Superconductivity : Science, Technology, and Applications. 2009, Weinheim: Wiley-VCH.

3. V.Shemelin and H.Padamsee, Magnetic field enhancement at pits and bumps on the surface of superconducting cavities. 2008-07.

4. Palmieri, V., et al. Besides the Standard Niobium Bath Chemical Polishing. in The 10th WOrkshop on RF Superconductivity. 2001. Tsukuba, Japan.

5. K.Saito, et al. Superiority of Electropolishing over Chemical Polishing on High Gradients. in 1997 Workshop on RF Superconductivity. 1997. Abano Terme (Padova), Italy.

6. Tian, H., et al., The mechanism of electropolishing of niobium in hydrofluoricsulfuric acid electrolyte. Journal of the Electrochemical Society, 2008. 155(9): p. D563-D568.

7. Eozenou, F., et al., Electropolishing of Niobium: best EP Parameters, in CAREReport-06-010-SRF. 2006. 


\section{Chapter 2 Literature Review}

\subsection{Introduction}

High energy physics studies require the use of modern accelerators that can accelerate elementary particles like electrons and positrons to a velocity close to the velocity of light. A key component of these accelerators is a device that provides energy to the charged particle. The device used is an electromagnetic cavity resonating at radio frequencies [1]. In a number of present day machines, copper cavities are used. However, higher energy machines require superconducting materials like niobium to make the cavities because of its low surface impedance in the superconducting state. The performance of these superconducting radio frequency (SRF) cavities is characterized by the accelerating gradient they can provide to the charged particles. Achieving the best performance requires the surface of these cavities to be free from defects and contaminants. Pits, bumps and protrusions severely limit the performance of the SRF cavities [2]. The SRF cavities are formed by deep drawing niobium sheets and then electron beam welding (EBW) at the equator and the iris as shown in Fig. 2.1. The Heat Affected Zone (HAZ) of the EBW region is especially prone to defects after electropolishing [3]. Therefore, a surface treatment is required to remove about 100-150 $\mu \mathrm{m}$ of damaged surface layer [1]. There are many ways to lower the surface roughness 
and reduce the defects on the surface of the SRF cavities. Chemical etching called buffered chemical polishing (BCP) involves etching of the niobium surface with a 1:1:1 or 1:1:2 mixture of $\mathrm{HF}, \mathrm{HNO}_{3}$ and $\mathrm{H}_{3} \mathrm{PO}_{4}[4,5]$. Centrifugal barrel polishing (CBP) also called tumbling is another surface treatment method involving the rotation of the cavities at high speed with abrasive media [6]. Electropolishing of niobium surface is another method, and one that has been found to be the most effective surface treatment for achieving consistent accelerating results [7]. The SRF cavities are processed by filling about $50 \%$ of their volume with electrolyte [8]. An aluminum counter electrode in the form of a rod is passed through the iris of these cavities. The ends are then sealed to provide a leak proof assembly. The SRF cavity is connected to the positive end of the power supply while $\mathrm{Al}$ acts as the negative terminal. Fig. 2.2 shows an electropolishing set-up for single cell cavity processing at Fermilab [9]. In general, the cavity is rotated at rates which varies between laboratories depending on their processing. The aim of this literature review is to address the present understanding of the mechanism of electropolishing, the surface phenomena taking place and the defect formation mechanism. Some attention also will be given to the phenomenon of electropolishing at the pit bottoms in classical corrosion.

\subsection{Fundamentals of electropolishing}

Electropolishing is a surface finishing process by which a metal surface is made smooth and bright by making it an anode in an appropriate electrolytic solution [10, 11]. There 
are many applications of electropolishing, for example in surgical tubing, medical cutting instruments, food processing blades, hard disk drives and automotive parts [12].

The earliest explanations of electropolishing were given by Jacquet and Elmore. Jacquet attributed electropolishing to the formation of a viscous layer on the workpiece [10]. The non-uniform thickness of the viscous layer over the material surface results in a different ohmic resistance from the cathode to the anode. This cause greater dissolution of the protruded parts compared to the depressed part, thus creating a uniform surface profile. This explanation was later challenged by Elmore who studied the current-voltage relationship for the polishing of copper in orthophosphoric acid system. He attributed the polishing effect to a varying concentration gradient of the dissolved metal ions over the protrusions and valleys as shown in Fig. 2.3 [13]. The value of the diffusion layer thickness, $\delta$ over the protrusion is smaller than the value over the valley. Therefore, the value of the limiting current defined by equation 1 would be larger over the protrusion compared to that over the valley, resulting in the metal surface being leveled out as electropolishing proceeds. This is the generally accepted mechanism for electropolishing and mass transport control has been established as the reason behind electropolishing. Electropolishing of many metals was studied henceforth and it was found a particular voltage range in the I-V characteristics of the metal in the electrolytic system is necessary to get the best surface finish. This voltage range is associated with mass transport control and is characterized by a constant current density independent of the voltage applied. The limiting current density $\left(\mathrm{i}_{\mathrm{L}}\right)$ is given by the following relation [14]. 


$$
i_{L}=\frac{n F D\left(C_{s}-C_{b}\right)}{\delta}
$$

$\mathrm{n}=$ charge of the ion involved

$\mathrm{F}=$ Faraday constant

$\mathrm{D}=$ Diffusion coefficient of the rate limiting species.

$\mathrm{C}_{\mathrm{s}}=$ Surface concentration (saturation concentration of metal ions in the solution when a salt film mechanism is taking place or zero when an acceptor mechanism is taking place which is detailed later in the review)

$\mathrm{C}_{\mathrm{b}}=$ Bulk concentration (which is approximately zero when salt film mechanism is taking place or is the bulk concentration of the acceptor species when acceptor mechanism is involved)

$\delta=$ Thickness of the Nernst diffusion layer $[15,16]$.

In the Nernst diffusion layer model, it is assumed that a concentration gradient exists at the interface which varies linearly initially and then has a curvature and merges with the final constant bulk concentration of the species under consideration [16]. It is a mathematical construct that separates the electrolyte region near the surface in which transport is by diffusion from rest of the electrolyte.

The mechanism of electropolishing is related to the current distribution on the surface of the sample. The current distribution has been classified as primary, secondary, tertiary or 
quaternary depending on the factors influencing it [17]. The primary current distribution is affected by the ohmic resistance and its role in electropolishing is dependent on geometrical factors like anode-cathode distance and initial geometrical profile of the surface to be polished. The secondary current distribution includes the effect of charge transfer overpotential along with the geometrical factors. Concentration overpotential along with the above factors constitutes the tertiary current distribution and is a necessary condition to obtain a smooth surface by electropolishing. Hydrodynamic effects also contribute to the current distribution. Hydrodynamics has been found to be an important consideration for defects generated during electropolishing [18].

\subsection{Different regions of electropolishing polarization curve}

A typical polarization curve for an electropolishing system looks like Fig. 2.4. Four major regions can be identified on the curve: etching region, oscillation region, polishing region and gas evolution region. The etching region is characterized by an exponential increase in current until a certain voltage, $\mathrm{V}_{\mathrm{b}}$. The Butler-Volmer kinetics given by equation 2 are followed in this region [19].

$$
i=i_{o}\left[\exp \left(\frac{\beta n F \eta}{R T}\right)-\exp \left(-\frac{(1-\beta) n F \eta}{R T}\right)\right]
$$

where $\eta$ is the overpotential, $\mathrm{E}-\mathrm{E}_{\mathrm{rev}}[20], \beta$ is the symmetry factor, $\mathrm{R}$ is the gas constant and $\mathrm{T}$ is the temperature, $\mathrm{n}$ and $\mathrm{F}$ are the same as in equation 1. 
It has been reported that sites such as grain boundaries and defects have different standard reversible potential than the metal surface and thus have a different value of overpotential, $\eta$, at a given overall applied potential, resulting in a different dissolution current given by equation 2 [21]. This results in crystallographic etching rather than polishing. The rate of transport of the anions to the surface also increases with voltage in the etching region. At $\mathrm{V}_{\mathrm{b}}$ current oscillation sets in, as further increase in voltage leads to a competition between the thickening viscous film and the increasing transport rate of anions to the surface. It becomes increasingly difficult for the anions to reach the surface because of the thickening viscous layer [22]. At $V_{c}$, the diffusion layer establishes a stable thickness and further increase in voltage to $\mathrm{V}_{\mathrm{d}}$ does not result in any increase in current density. The current peak before the current density plateau is also explained on the basis of super-saturation of the salt forming in case of the salt film mechanism of electropolishing. The film then equilibrates to the saturation level resulting in a drop of the current density to the limiting current density value [14]. The region from $V_{c}$ to $V_{d}$ is called the plateau region and the best electropolishing conditions are found in this region. The plateau is formed because of mass transport control. Application of potential above $\mathrm{V}_{\mathrm{d}}$ results in oxygen evolution on the metal surface along with the metal dissolution. Electropolishing in this voltage regime results in the surface being rougher because oxygen bubbles formed may attach to the surface and cause occlusion of the surface at certain locations resulting in differing dissolution rates [17]. The occluded area may contain electrolyte below the gas bubble resulting in etching. Depending on the system, the dissolution rate in the occluded area may be higher or lower than the remaining 
surface. Pitting results if the rate is higher and a bump is formed if the rate is lower. In either case a rough surface results if electropolishing is attempted in this potential region. It has also been reported that polishing can take place at potentials way above the $\mathrm{V}_{\mathrm{d}}$ potential. Palmieri et al. states that polishing occurs in this region because the rate of oxygen generation is so fast that oxygen bubbles leave the surface as soon as they are formed and hence do not get a chance to occlude the surface and cause pitting [22].

\subsection{Electropolishing mechanisms}

As stated above, the best polishing occurs in the current plateau region where mass transport limitation controls the current distribution. The mass transport limitation can occur by three mechanisms depending on the diffusion limited species, which can be the dissolving metal ions, acceptor ions or water molecules [17]. The first mechanism is called salt precipitation mechanism (Fig. 2.5-I) as a salt film gets precipitated on the metal surface by the reaction between the dissolving metal ions and the electrolyte anions. This film fixes the concentration of the metal ions at the anode to the saturation concentration. Thus, a constant concentration gradient exists across the diffusion layer, resulting in a constant current density [17]. Nickel electropolishing in sulfuric acid and iron dissolution in $4 \mathrm{M}$ sodium chloride solution have been found to follow this mechanism $[23,24]$.

The second mechanism is based on the diffusion limited approach of acceptor anions to the metal surface as shown in Fig. 2.5-II. The acceptor anions complex the dissolving metal ions to form a viscous layer on the surface. The surface concentration of the 
diffusion limited species is fixed to zero as they are consumed as soon as they reach the surface. Electropolishing of niobium in 1:9 volume ratio mixture of $\mathrm{HF}$ and $\mathrm{H}_{2} \mathrm{SO}_{4}$ has been shown to follow this mechanism [25]. Water is mass transport controlled in the third mechanism (Fig. 2.5-III). The water molecules are used up for hydrating the dissolving metal ions as soon as they reach the surface resulting in its surface concentration being fixed at zero under electropolishing conditions. Copper electropolishing in phosphoric acid has been shown to follow this mechanism [26]. Fig. 2.5 shows the idealized concentration profile that exists for the three mechanisms stated above.

\subsection{Reactions occurring during electropolishing of niobium}

The standard electrolyte used for electropolishing of niobium SRF cavities is a 1:9 volume ratio of hydrofluoric acid (48\%) and sulfuric acid (96-98\%). It was originally developed at Siemens by Diepers et al. [27] and was further improved upon at KEK Japan. Niobium has a thin stable oxide layer of $\mathrm{Nb}_{2} \mathrm{O}_{5-\mathrm{x}}$ [7]. This passive layer is unstable in the presence of fluoride ions and hence HF is indispensable for niobium electropolishing. The following reactions are expected to occur at the niobium working electrode (note that the second and third are generic reactions that are unbalanced) [7]:

$$
\begin{gathered}
2 \mathrm{Nb}+5 \mathrm{H}_{2} \mathrm{O} \rightarrow \mathrm{Nb}_{2} \mathrm{O}_{5}+10 \mathrm{H}^{+}+10 \mathrm{e}^{-} \\
\mathrm{Nb}_{2} \mathrm{O}_{5}+\mathrm{HF} \rightarrow \mathrm{H}_{2} \mathrm{NbOF}_{5}(\mathrm{aq})+\mathrm{HNbF}_{6}(\mathrm{aq})+\mathrm{NbF}_{5}(\mathrm{aq})+\mathrm{H}_{6} \mathrm{NbO}_{2} \mathrm{~F}_{7}(\mathrm{aq})+\mathrm{H}_{2} \mathrm{NbF}_{7}(\mathrm{aq})+ \\
\mathrm{H}_{2} \mathrm{O} \\
\mathrm{NbF}_{5}+\left(\mathrm{SO}_{3}, \mathrm{H}_{2} \mathrm{O}\right) \rightarrow \mathrm{NbF}_{3}\left(\mathrm{SO}_{3} \mathrm{~F}\right)_{2} \cdot 2 \mathrm{H}_{2} \mathrm{O}(\mathrm{aq})+\mathrm{Nb}_{2} \mathrm{O}\left(\mathrm{SO}_{4}\right)_{4}(\mathrm{aq})+\mathrm{S}_{2} \mathrm{O}_{5} \mathrm{~F}_{2}
\end{gathered}
$$


The reaction at the aluminum counter electrode is the hydrogen evolution reaction:

$$
2 \mathrm{H}^{+}+2 \mathrm{e}^{-} \rightarrow \mathrm{H}_{2}
$$

\subsection{Optimum voltage consideration for electropolishing}

The i-V characteristics of an electropolishing system depends on many factors including orientation of anode and cathode in the bath, cathode material used, anode to cathode surface area ratio, electrolytic bath age, temperature of the electrolyte and degree of agitation [11]. Therefore, the $\mathrm{i}-\mathrm{V}$ relationship should be ascertained for the actual situation. For example, before electropolishing of SRF cavities, the characteristic current density vs cell voltage polarization curve should be determined with the actual cavity arrangement as the polarization curves for the complex shaped SRF cavities may be different than those obtained for niobium coupons. The electropolishing is usually done by applying a voltage on the plateau. However, in practice the best polishing results are obtained by applying a voltage just below the potential at which the oxygen evolution starts [22]. An early explanation for this was proposed by Hoar et al. [28] who related it to the film that forms on the surface while electropolishing. The dissolution of metals results in formation of cations that move through the film to the solution, leaving vacancies at the metal-film interface. If the vacancy concentration increases too much, they could merge and form voids that might lead to the detachment of the ion conducting film from the surface. To avoid this, the potential should be kept as high as possible while ensuring that anions in the film are kept pressed against the metal surface in the receding metal-film interface. Antoine in a tutorial said that Teggart reported that the best voltage 
is the one when the cell impedance is the highest i.e., when V/I is maximum [29]. The value of $i$ is dependent on the temperature and composition of the electrolyte among other factors. In practice, the composition of the electrolyte keeps changing as the anions get consumed and the metal ions enter the solution, thus changing $\mathrm{i}$ and hence V/I. Ideally the optimal V should be adjusted throughout the electropolishing duration, but due to practical reasons, it is chosen at the value just below oxygen evolution. The importance of conducting electropolishing at a voltage just below the oxygen evolution potential has also been related to the nature of the current density vs. time curve at the start of the polishing. A typical curve would look as shown in Fig. 2.6. As the electropolishing potential is decreased, more charge is passed before the stable current density is obtained. It has been reported that less charge should be passed in this initial phase to obtain a good surface finish [30].

\subsection{Surface phenomena occurring during electropolishing}

Understanding the surface processes occurring on the metal surface during electropolishing can provide key insights into its mechanism. This can help in optimizing the parameters in an informed manner rather than by trial and error approach. Electrochemical Impedance Spectroscopy (EIS) [31-34], galvanostatic transient technique and rotating disk electrode method are key electrochemical tools that help in investigating the electropolishing mechanism of a particular metal-electrolyte system. The use of the electrohydrodynamic impedance technique has also been reported by West et al [35]. 
Matlosz [31] gave an account of the nature of the surface phenomena taking place during electropolishing. As stated earlier, mass transport limitation is the cause of electropolishing and the diffusion limited species could be the dissolving metal ion, an acceptor species, which is an ion from the electrolyte, or water molecules. When the diffusion limited species is the metal ion, a salt film precipitates on the surface, the nature of which could either be compact, porous or duplex nature containing both compact and porous components. Fig. 2.7 shows the schematic representation of the duplex salt film model as proposed by Matlosz for the electrodissolution of $\mathrm{Fe}$ in chloride solution. The mechanism of conduction is stated to be solid state conduction in the compact layer and the conduction in the porous region is by migration in the electric field. There is no diffusion here as it is assumed that the pores are filled with the electrolyte saturated with the dissolved metal ions. The high resistance of these films is explained on the basis of lower porosity of the porous film and its large thickness on the order of several microns. The high resistance of the compact film is attributed to low mobility of ions for solid state transport. The thickness of the compact film is considered to be in the order of $10 \mathrm{~nm}$, again due to the low mobility of ions by solid state transport process. The limiting current density is finally decided by the concentration gradient of the metal ions across the diffusion layer.

Hoar et al. made suggestions regarding the nature of the surface film formed during electropolishing [28]. They postulated that the film formed on a surface undergoing dissolution is not a simple oxide but rather a contaminated oxide that has incorporated anions from the electrolytic solution. It is hypothesized that such an oxide could account 
for better ion conductivities than simple oxides. Vetter et al. in their study on pitting corrosion emphasized that salt layer formation takes place at the bottom of pits and there is an ionic transport through this layer because of the presence of a high electric field across the layer [36]. Strehblow et al. also made certain conclusions regarding the nature of the film formed during electrodissolution of iron and nickel in high chloride media [37]. They justified the presence of a compact film on the surface during electropolishing owing to the fact that gas bubble evolution does not occur on the surface even though the potentials are high enough to thermodynamically allow bubble formation. They argue that this is due to the non-electron-conducting nature of the film. They, however, do not discuss the transport mechanism, which supports the polishing current and it can be by high field mechanism. Strehblow's analysis may be true for some electropolishing systems but gas evolution has been found in others.

Matlosz also postulated the phenomena taking place at the surface when the current density are controlled by the diffusion limited acceptor ion approach to the surface [31]. Fig. 2.8 shows the schematic representation of the adsorbate acceptor model. The dissolution proceeds by the oxidation of the metal, $\mathrm{M}$ which form cations that get adsorbed onto the surface. The author attributed the overpotential for metal dissolution to the blocking of the surface by these adsorbed ions. The cations are removed from the surface by the acceptor ions that diffuse to the surface to solvate the metal ions. At steady state, the concentration of the acceptor ions at the surface is fixed at zero assuming that they are consumed as soon as they reach the surface. Matlosz says that a film does not 
exist in this mechanism and justifies it by assuming that acceptor ions do not have sufficient mobility in a solid phase.

Recent papers on the study of copper electropolishing in phosphoric acid and niobium electropolishing in sulfuric acid-hydrofluoric acid mixture have shown that an acceptor species is the rate limiting species that diffuses to the surface $[25,26,36,38]$.

Galvanostatic experiments can provide important insights into the nature of the surface phenomena occurring during electropolishing. The potential transients measured for copper electropolishing system [36] showed a sharp rise in potential on applying the current which is due to the ohmic potential drop. It was followed by a potential increase because of activation overpotential and then a gradual increase owing to mass transport overpotential. There was a huge jump in potential after a certain time called the transient time $(\tau)$ indicating a very high resistance that can not be attributed to ohmic losses in solution or overpotential for oxygen evolution. The author interpreted it as the existence of a highly resistive surface film. But that potential jump could be because of water concentration going to zero at the electrode surface as has been recognized as the electropolishing mechanism for copper by $\mathrm{Du}$ et al [26].The transient time is given by Sand's equation (equation 3) given below.

$$
\sqrt{\tau}=\frac{n F}{2 i} C_{\text {acceptor }}^{o} \sqrt{\pi D_{\text {acceptor }}}
$$


Where $\mathrm{C}_{\text {acceptor }}^{\mathrm{o}}$ is the concentration of the acceptor species in the bulk electrolyte, $\mathrm{D}_{\text {acceptor }}$ is the diffusion coefficient of the acceptor species, $\mathrm{F}$ is Faraday constant, $\mathrm{n}$ is the ion charge, $i$ is the applied current density.

Padhi et al. have concluded the electropolishing mechanism for copper in phosphoric acid considering the validity of Sand's equation [38]. The interpretation should be made keeping in mind the basis of derivation for the equation. The equation is based on the semi infinite linear diffusion model [39]. The process of electropolishing could result in the density near the electrode different from the bulk of the solution resulting in a hydrodynamic flow called natural convection [40]. Sand's equation given above does not take into account the convection with diffusion mechanism and hence the calculations based on it should be treated accordingly.

\subsection{Phenomenon of electropolishing of pit bottoms in pitting corrosion}

The phenomenon of electropolishing and the importance of salt films have been studied with respect to pitting corrosion where the pit bottoms are often found to be electropolished. Electropolished pit bottoms appear for a wide range of metals and alloys based on iron, nickel, titanium, tantalum and cobalt exposed to chloride solutions and so is a very general phenomenon like electropolishing [41]. Pitting corrosion is a form of corrosion where high corrosion currents develop on a passive metal surface at some localized regions due to the breakdown of the passive film [42]. The electrochemical polarization curves for the phenomenon of anodic passivity and electropolishing are very similar. They differ in the current density magnitude order with passivity showing a 
current density plateau of magnitude of about $10^{-6}-10^{-10} \mathrm{~A} / \mathrm{cm}^{2}$ and electropolishing having a much higher value of more than $10 \mathrm{~mA} / \mathrm{cm}^{2}$. Strehblow et al. stated that electropolishing involves the prevention of passivation of the entire metal surface in a fashion similar to pitting where only local breakdown of passivity is involved in the presence of high chloride concentration [37]. Precipitation of salt films has been reported during the initial stages of pitting corrosion due to high local current densities at the site of pits [43]. The corrosion rate in the pit was found to be controlled by mass transfer processes as is the case in electropolishing. The precipitation of the salt film has been found to be important for the stability of the pits as the film provides the necessary voltage drop to keep the potential of the metal in the pit to be in the active region while the rest of the metal surface is in the passive region [44]. However, Frankel has suggested that they are not essential for the formation of a stable pit [42]. Frankel et al. in their model have emphasized the importance of salt films for the pit stability bringing into consideration the importance of a pit cover as well. They state that the tendency for a metastable pit to become stable is enhanced if a salt film precipitates on the pit surface before the pit cover breaks [45].

A duplex salt film precipitation has been proposed for film formation in pits similar to that reported by Maltosz for the electropolishing process. Vetter et al. found a compact layer of $\mathrm{FeCl}_{2}$ precipitated on top of which a porous layer of $\mathrm{FeSO}_{4}$ is precipitated in pits formed in Fe in solutions containing sulfate and chloride ions [46]. The hemispherical growth and electropolishing of the pits is attributed to the statistical fluctuations of the pores in the sulfate layer leading to random dissolution of the metal, thus preventing 
etching. Hoar et al. explained the electropolishing phenomenon on similar lines to fluctuations of pores [28]. Strehblow and Wenners later also propose a similar mechanism for electropolishing of nickel in chloride solutions [46]. They believe that a compact film forms on the anode surface which prevents etching of metal, as the mechanism dictating metal dissolution is the random removal of metal ions from the surface due to fluctuating pores in the compact layer. This explanation is somewhat similar to the point defect model for passive film growth of passive film first proposed by Chao et al [47]. The metal vacancies form in the film as it has a stable thickness at a particular potential and there is a steady rate of dissolution of film, which is equal to the rate of formation. The vacancies form at the film/electrolyte interface and move to the metal/film interface by diffusion and migration.

\subsection{Phenomenon of pitting in anodic dissolution of metals and alloys}

Electropolishing is undertaken to obtain a smooth surface finish that is critical to the application handoff SRF cavity processing. However, electropolished surfaces are not always free of defects and problems. Pitting of the surface is one major problem that results in the lowering of the performance expected of the electropolished surface. For example, in the case of electropolishing of $\mathrm{Nb}$ used for SRF cavities, the accelerating gradients achievable are deteriorated because of the presence of these surface defects. The process of pitting in the case of electropolishing is different from the conventional pitting, which takes place due to increased local dissolution due to breakdown of protective passive film. The application of current and voltage on the polishing plateau is 
a necessary condition to obtain a pit free surface but not a sufficient condition. A multitude of reasons have been attributed to be responsible for pitting some of which include (i) oxygen evolution at the surface getting polished, (ii) hydrogen evolved on the cathode, (iii) inclusions on the surface, (iv) stresses on the surface, (v) excess agitation in the polishing bath, and (vi) external treatments given to the surface [3, 4, 28, 48-54].

\subsubsection{Role of oxygen evolution in defect formation}

Application of voltage much greater than the reversible potential for oxygen evolution can result in oxygen generation on the surface to be polished. The oxygen bubbles formed can result in pitting [55] by two ways. The viscous film gets disrupted when the generated oxygen bubble leaves the metal surface resulting in localized attack of the metal surface and hence pitting. The oxygen bubble may also occlude the surface just below it and hence result in more dissolution of the surrounding surface and formation of defects. This problem has been highlighted in copper electropolishing. This problem can be avoided by either preventing the oxygen bubbles from sticking to the surface or increasing the overpotential for oxygen production. Shieh et al. proved the reduction of pit formation during electropolishing of $\mathrm{Cu}$ in $\mathrm{H}_{3} \mathrm{PO}_{4}$ by addition of polyethylene glycol (PEG) and sulfuric acid [53]. PEG adsorbed on the copper surface resulting in an increased charge transfer resistance and increase in the overpotential for oxygen formation. The sulfuric acid resulted in an increased resistance of the viscous film formed with effects similar to the PEG addition. The combination of PEG and sulfuric acid resulted in lower contribution of oxygen evolution reaction current to the polishing 
current and hence lower pitting on electropolished copper surface. Palmieri et al. suggested that the use of a potential far above the oxygen evolution potential results in rapid formation of oxygen bubbles that cannot adhere to the surface because of rapid evolution and hence pitting is avoided [4]. This approach to combat pitting might result in other problems like a very high dissolution current resulting in heat generation. This in turn would result in increased temperature, causing the properties of the viscous layer to change and also a varying hydrodynamics i.e. convection [3]. The control of these properties is crucial to attain a good finished surface as they are critical to achieve a mass transport control, which is required for the phenomenon of electropolishing. A way to prevent oxygen from adhering to the surface could be the use of a controlled agitation of the electrolyte to facilitate the removal of the oxygen generated from the working electrode surface [54]. However, agitation should be very carefully controlled in order not to disturb the viscous layer; otherwise all the problems stated above would apply here as well.

\subsubsection{Role of hydrogen evolution at cathode on pitting}

Hydrogen evolution is the reaction which usually takes place at the counter electrode during electropolishing. The hydrogen generated can travel to the working electrode through the electrolyte where it can cause pitting by a mechanism similar to that explained above for oxygen bubbles [54] or by hydride formation [56]. The problem of pitting because of hydrogen bubble generated at the cathode has been present in the aluminum EP system [54]. It resulted in doughnut shaped pits because of the occlusion of 
the surface below the bubble and increased attack in the surrounding reasons. The problem was addressed with a careful agitation of the electrolyte to remove the hydrogen bubbles. It has been reported in the case of chemical polishing of niobium that hydrogen diffuses through the oxide layer on its surface forming niobium hydride precipitates (Nb4H3-x) [56]. The hydride has a slightly higher density than niobium. This results in a decrease in volume when the hydride precipitates, causing formation of dislocation to account for the mismatch between the precipitate and the matrix. Dislocation etch pits are formed in such cases. A similar mechanism for pitting may take place during EP of niobium as well but this time the hydrogen absorption into the material takes place at times when the metal is in the electrolytic solution without the voltage bias [57]. The solution to the problem could be preventing the hydrogen generated from reaching the anode surface while maintaining the ionic conductivity of the electrolytic solution. The approach used in the polishing of SRF cavities has been to flow nitrogen in the space between the electrolyte and the SRF cavities [3]. The nitrogen flow carries away the hydrogen generated. This however cannot totally solve the problem as there are still dissolved hydrogen bubbles in the electrolytic bath that can reach the niobium surface and cause pitting as explained by Higuchi et al [57].

\subsubsection{Other factors resulting in pitting during electropolishing}

Stress induced in the metal has also been related to the cause of pitting. It has been reported by Frankenthal that anodic dissolution of $\mathrm{Fe}-\mathrm{Cr}$ alloys results in higher pit growth rate when the starting material is mechanically polished than when the material 
has been given a brief electropolishing treatment. This was related to the plastic deformation caused in the material because of mechanical polishing. This deformation results in formation of active dislocations, which becomes sites for pitting as the atoms at these locations are loosely held because of lower bonding energies [58]. Cooley et al. compared the pit formation after electropolishing of as received recrystallized niobium coupon (AR), cold worked coupon (CW) and cold worked coupon which was annealed (CW-AN). They found that the AR coupons had the least population of pits followed by CW-AN sample and the CW coupons had the highest pit population. Higher dislocation density resulted in higher dislocation etch pits. Pitting has been found to be also related to welding and the HAZ region is prone to pitting which can be due to pile up of dislocations [3].

Electropolishing of alloys presents the problem of pitting at the site of inclusions. Imboden et al. have reported this problem in the case of electropolishing of plain carbon steel [50]. The viscous film necessary for electropolishing is not well formed at the site of inclusions, resulting in greater attack in the locality and hence in pitting. The method suggested to tackle the problem was agitation of the electrolyte to keep the viscous film thin at other places as well which will result in higher dissolution current at other places as well. This approach would not solve the problem of pitting completely. It would although make it less intense [50]. Impurities or inclusions introduced in the EBW region of SRF cavities also present similar problems of pitting for EP of niobium [48]. Sulphide inclusions in nickel have been found to be the site where pits nucleate during anodic dissolution of nickel [49]. 
Geometry of the surface getting electropolished needs to be considered as well. If the geometry is such that the formation of a stable viscous layer necessary to attain EP cannot be formed, the mass transport control conditions are no longer available resulting in etching and pitting of the surface instead of EP. Such a situation is found for a downward facing surface on which viscous layer can not support its own weight and falls down under the effect of gravity resulting in disruption of the diffusion layer regime and hence no electropolishing [18]. 


\subsection{Figures:}

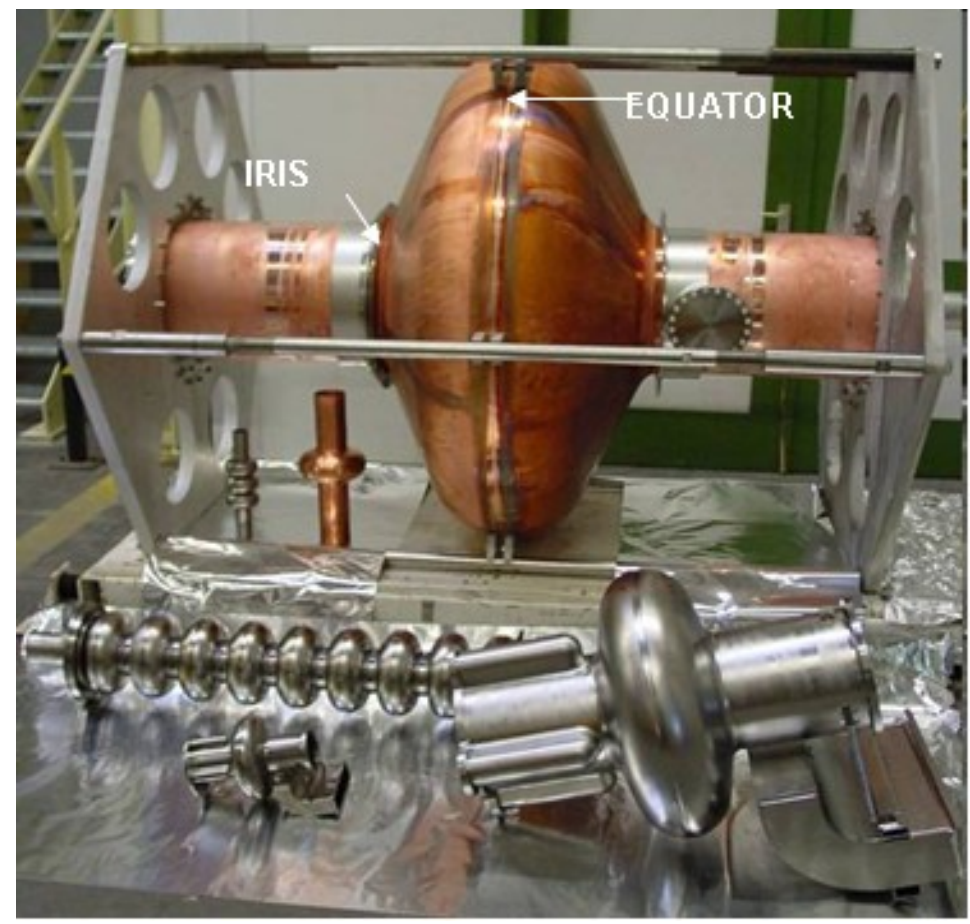

Fig. 2.1: SRF cavities [59]. 


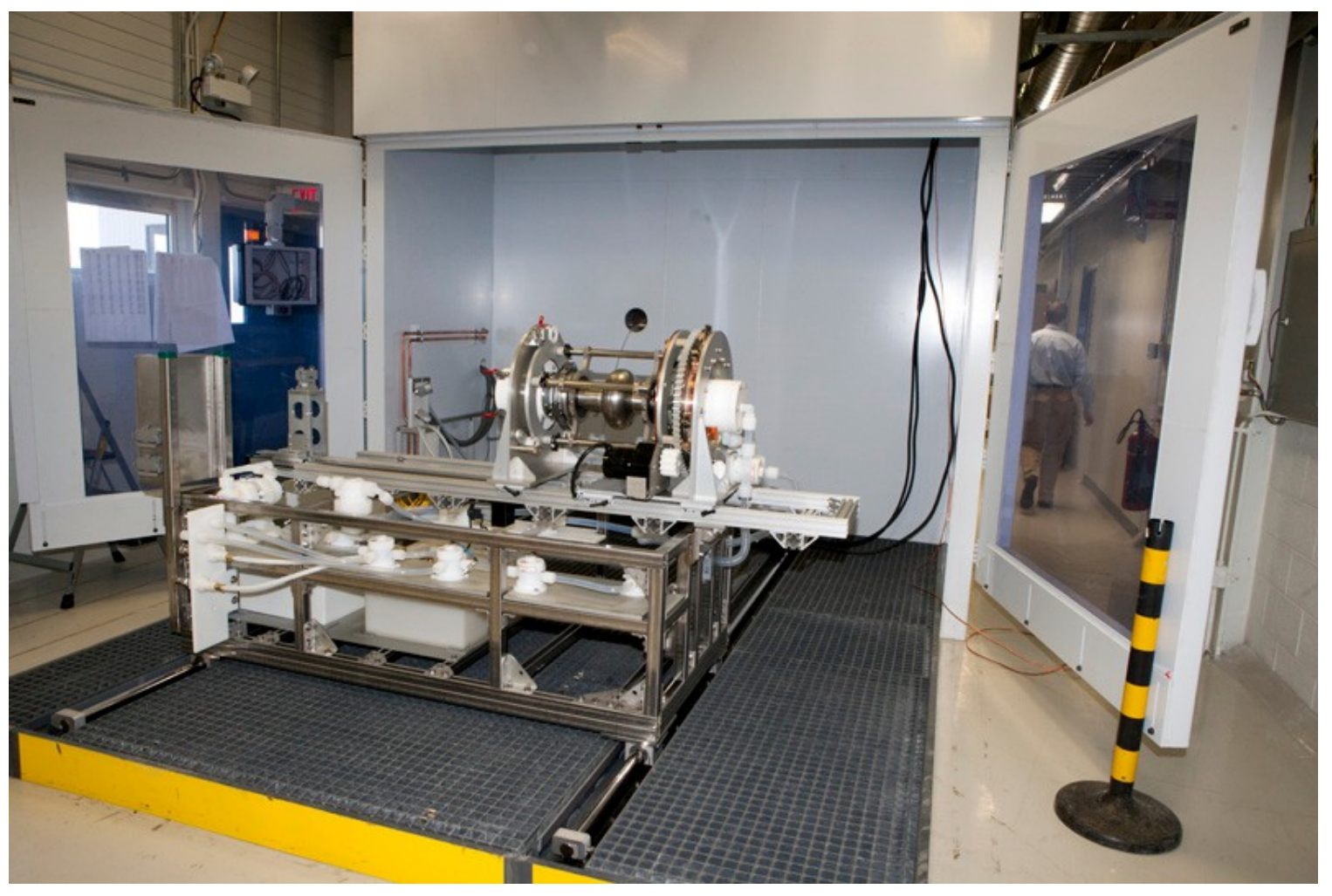

Fig. 2.2: Electropolishing set up at Fermilab showing an assembled niobium single cell cavity [9]. 


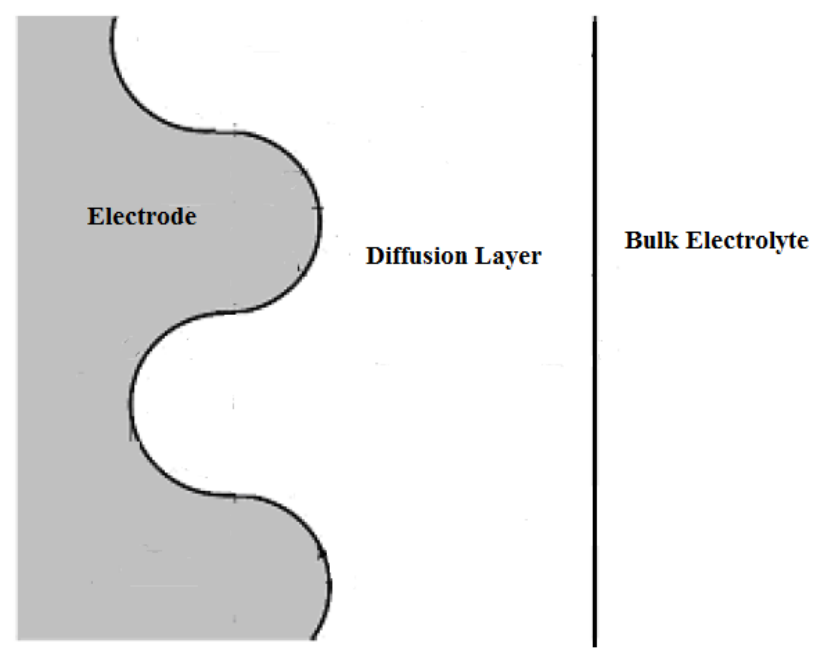

Fig. 2.3: Concentration gradient difference over protrusions and valleys [60]. 


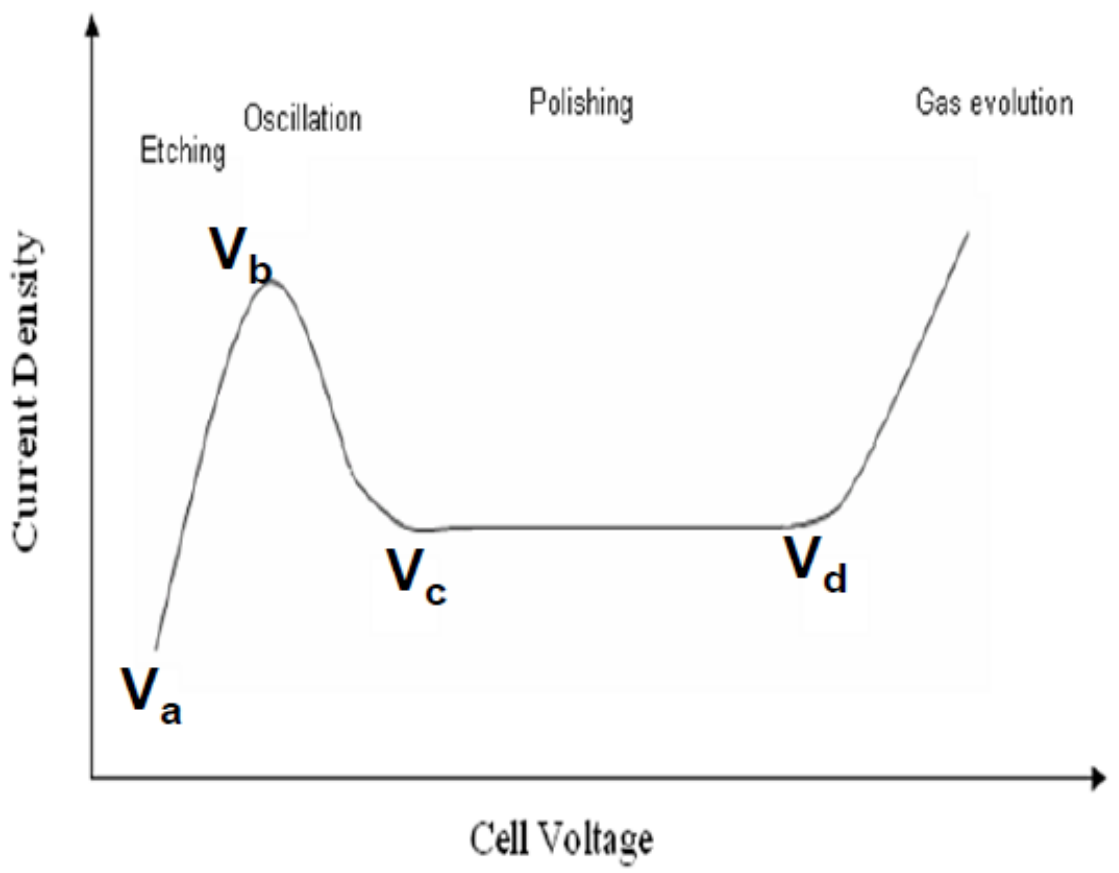

Fig. 2.4: A typical polarization curve for electropolishing systems. 


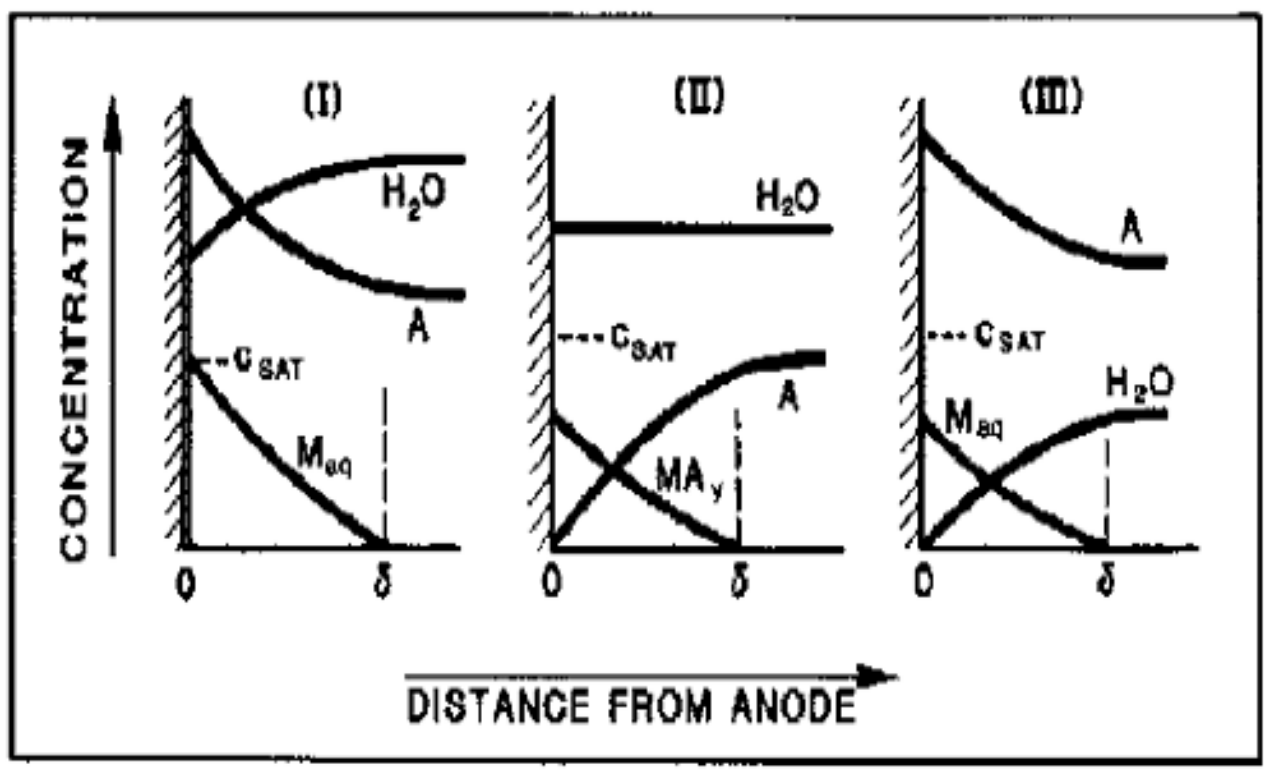

Fig. 2.5: Concentration profile of various species for the three electropolishing mechanisms [17]. 


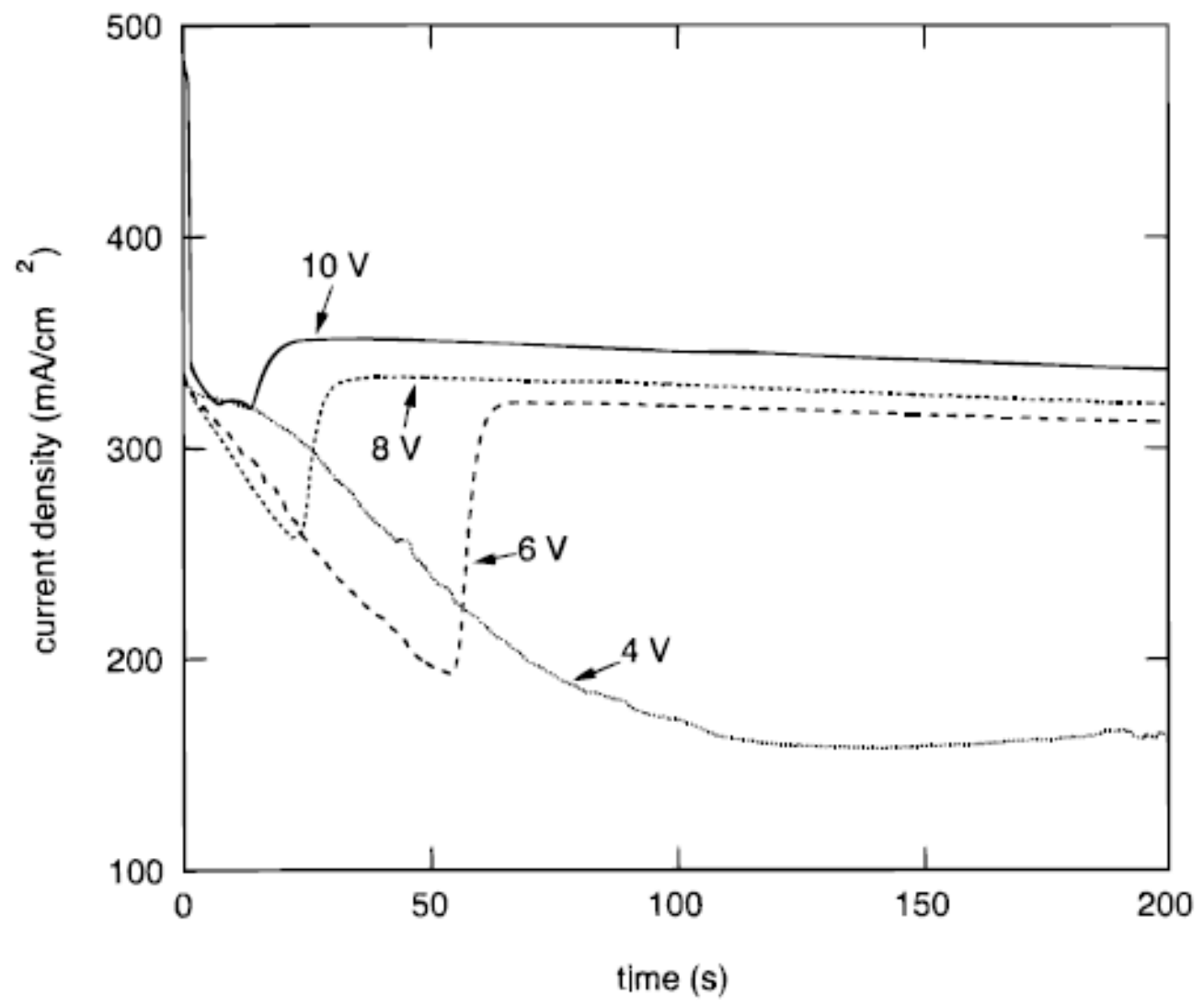

Fig. 2.6: i Vs t curve measured for Ti in 3M methanol-sulfuric acid electrolyte [61]. 


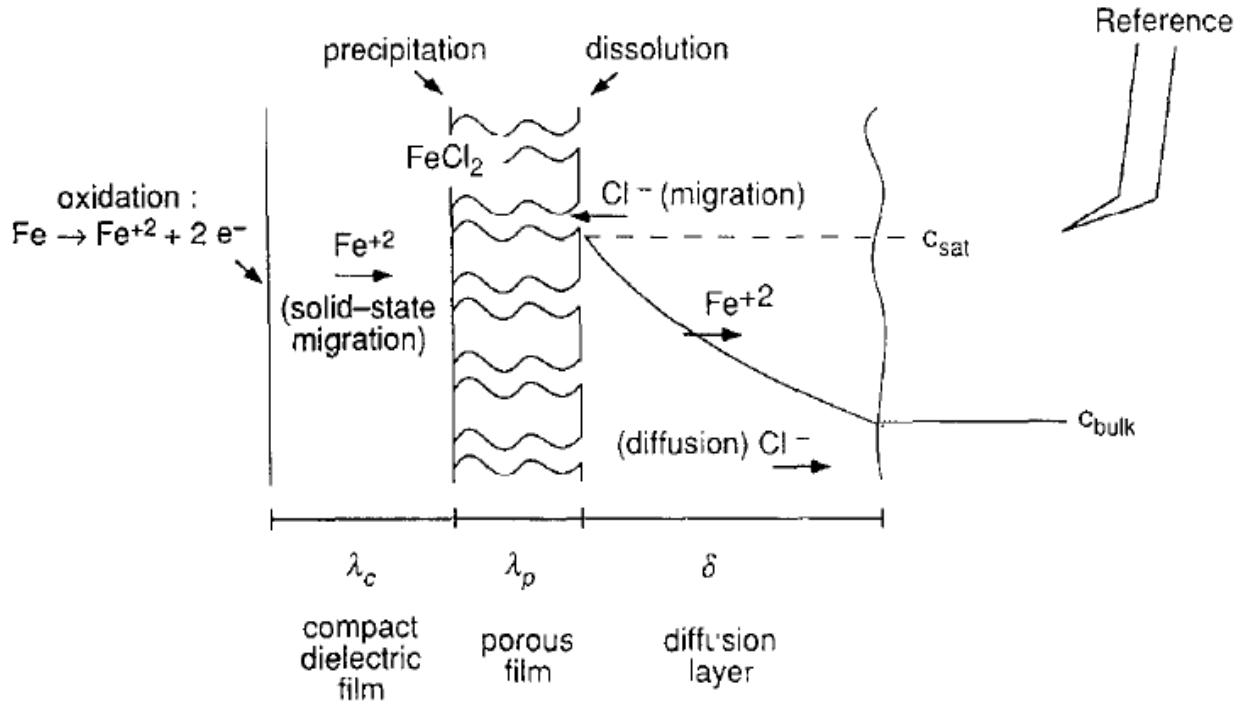

Fig. 2.7: Duplex salt film model schematic representation [31] 


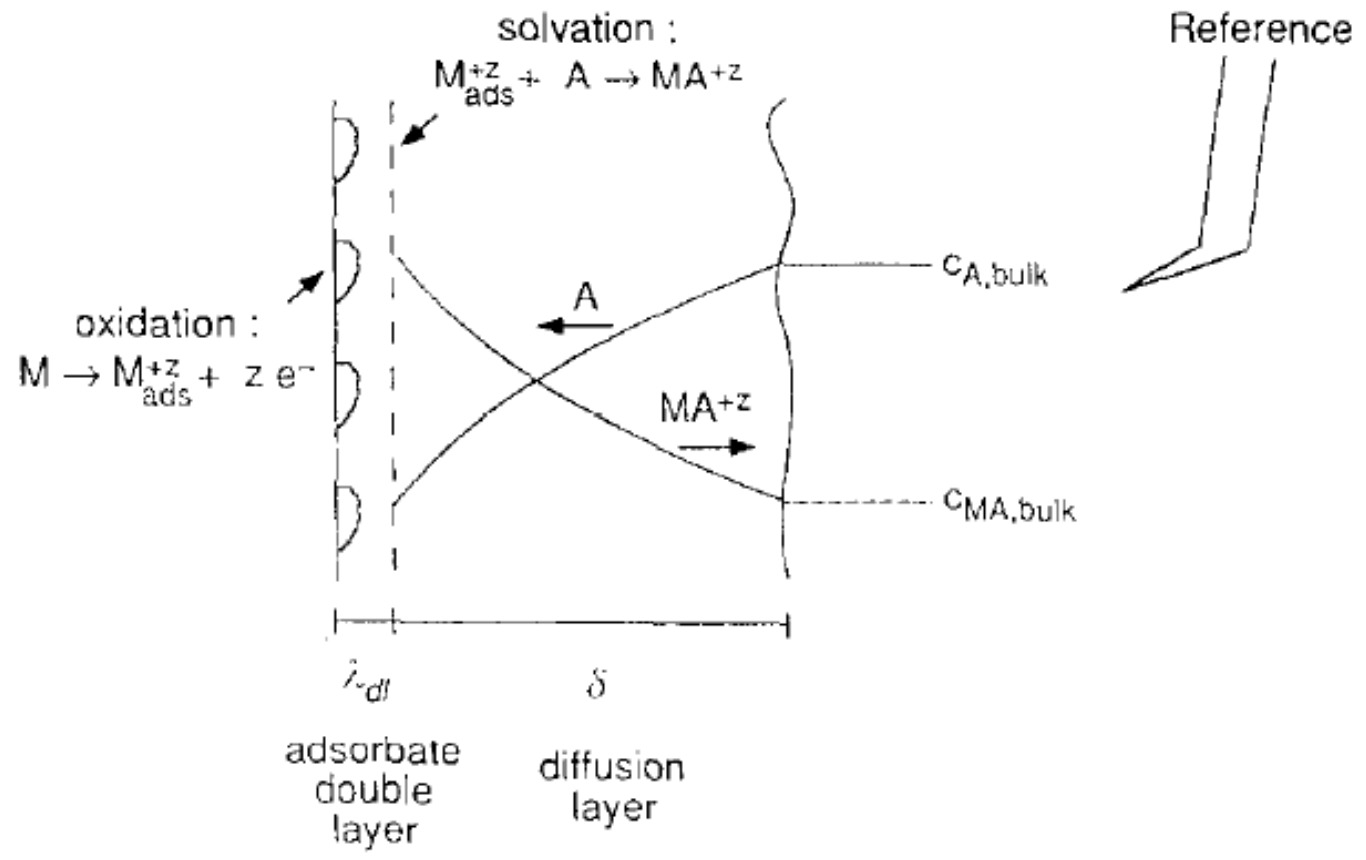

Fig. 2.8: Schematic representation of adsorbate acceptor model in EP [31]. 


\subsection{References}

1. H.Padamsee, J.Knobloch, and T.Hays, RF Super-conductivity for Accelerators. 1998, New York: John Wiley \& Sons.

2. V.Shemelin and H.Padamsee, Magnetic field enhancement at pits and bumps on the surface of superconducting cavities. 2008-07.

3. Cooley, L., et al., Impact of Forming, Welding, and Electropolishing on Pitting and the Surface Finish of SRF Cavity Niobium. 2010, Fermilab.

4. Palmieri, V., et al. Besides the Standard Niobium Bath Chemical Polishing. in The 10th WOrkshop on RF Superconductivity. 2001. Tsukuba, Japan.

5. K.Saito, et al. Superiority of Electropolishing over Chemical Polishing on High Gradients. in 1997 Workshop on RF Superconductivity. 1997. Abano Terme (Padova), Italy.

6. T.Higuchi, et al. Investigation on Barrel Polishing for Superconducting Niobium Cavities. in 1995 Workshop on RF Superconductivity. 1995. Gif-sur-Yvette, France.

7. Eozenou, F., et al., Electropolishing of Niobium: best EP Parameters, in CAREReport-06-010-SRF. 2006.

8. K.Saito. Development of Electropolishing Technology for Superconducting Cavities. in Particle Accelerator Conference. 2003. Portland, Oregon.

9. Fermilab. [cited; Available from: http://www.fnal.gov/pub/today/images10/100505-09D.jpg. 
10. P.A.Jacquet, Electrolytic Method for obtaining Bright Copper Surfaces. Nature, 1935. 135(3426): p. 1076.

11. Chemical and Electrolytic Polishing, in ASM Handbook. 2004, ASM International. p. 281-293.

12. [cited; Available from: http://www.ableelectropolishing.com/0905able.pdf.

13. W.C.Elmore, Electrolytic Polishing. Journal of Applied Physics, 1939. 10: p. 724727.

14. Frankel, G., MSE 735 Kinetics II. 2010, Fontana Corrosion Center: Columbus

15. Bockris, J.M. and A.K.N. Reddy, Modern Electrochemistry-Ionics. Vol. 1. 2002, New York: Kluwer Academic Publishers. 1232-1234.

16. Bard, A.J. and L.R. Faulkner, Electrochemical Methods: Fundamentals and Applications. 1980, New York: John Wiley \& Sons.

17. Landolt, D., Fundamental aspects of electropolishing. Electrochimica Acta, 1987. 32(1): p. 1-11.

18. A.Chandra, G.S.Frankel, and M.D.Sumption. Electropolishing of Niobium to Obtain Defect Free Surface. in SRF 2011. 2011. Chicago, USA.

19. Frankel, G., MSE 735 Kinetics I. 2010, Fontana Corrosion Center: Columbus.

20. Landolt, D., Corrosion and surface chemistry of metals. 2007, Lausanne, Switzerland: EPFL Press.

21. A.J.Bard and L.R.Faulkner, Electrochemical Methods Fundamentals and Applications. 2 ed. 2001, New York, USA: John Wiley \& Sons. 
22. Palmieri, V. Fundamentals of Electrochemistry - The Electrolytic polishing of Metals: Application to Copper and Niobium. in SRF Workshop 2003. 2003. Germany.

23. H.C.Kuo and D. Landolt, Rotating-disk electrode study of anodic dissolution of iron in concentrated chloride media. Electrochimica Acta, 1975. 20(5): p. 393399.

24. D.J.Schiffrin, The influence of mass-transfer on the mechanism of electropolishing of nickel in aquesous sulfuric acid. Electrochimica Acta, 1982. 27(7): p. 837-845.

25. Tian, H., et al., The mechanism of electropolishing of niobium in hydrofluoricsulfuric acid electrolyte. Journal of the Electrochemical Society, 2008. 155(9): p. D563-D568.

26. Du, B. and I.I. Suni, Mechanistic studies of $\mathrm{Cu}$ in Electropolishing in Phosphoric Acid Electrolytes. Journal of the Electrochemical Society, 2004. 151(6): p. C375C378.

27. Diepers, H., et al., A New Method of Electropolishing Niobium. Physics Letters, 1971. 37A(2): p. 139-140.

28. Hoar, T.P., D.C. Mears, and G.P. Rothwell, The Relationships between anodic passivity, brightening and pitting. Corrosion Science, 1965. 5: p. 279-289.

29. Antoine. EP-Tutorial. [cited; Available from: http://ilcdms.fnal.gov/Members/tajima/References/Antoine EP tutorial 01JUN2006.ppt/f ile view. 
30. Landolt, D., P.F. Chauvy, and O. Zinger, Electrochemical micromachining, polishing and surface structuring of metals: fundamental aspects and new developments. Electrochimica Acta, 2003. 48(20-22): p. 3185-3201.

31. Matlosz, M., MODELING OF IMPEDANCE MECHANISMS IN ELECTROPOLISHING. Electrochimica Acta, 1995. 40(4): p. 393-401.

32. Glarum, S.H. and J.H. Marshall, The Anodic Dissolution of Copper into Phosphoric Acid. Journal of the Electrochemical Society, 1985. 132(12): p. 28782885.

33. Vidal, R. and A.C. West, Copper Electropolishing in Concentrated Phosphoric Acid I. Experimental Findings. Journal of the Electrochemical Society, 1995. 142(8): p. 2682-2689.

34. Vidal, R. and A.C. West, Copper Electropolishing in Concentrated Phosphoric Acid II. Theoretical Interpretation. Journal of the Electrochemical Society, 1995. 142(8): p. 2689-2694.

35. A.C.West, et al., Electrohydrodynamic impedance study of anodically formed salt films on iron in chloride solutions. Journal of Electroanalytical Chemistry, 1992. 330(1-2): p. 693-706.

36. Mendez, J., et al., A Mechanistic Model for Copper Electropolishing in Phosphoric Acid. Journal of the Electrochemical Society, 2008. 155(1): p. D27D34. 
37. Strehblow, H.H. and J. Wenners, Investigation of the Processes on Iron and Nickel Electrodes at High Corrosion Current Densities in Solutions of High Chloride Content. Electrochimica Acta, 1976. 22: p. 421-427.

38. Padhi, D., et al., Planarization of Copper Thin Films by Electropolishing in Phosphoric Acid for ULSI Applications. Journal of the Electrochemical Society, 2003. 150(1): p. G10-G14.

39. Bard, A.J. and L.R. Faulkner, Electrochemical Methods Fundamentals and Applications. 2 ed. 2001, Hoboken, NJ: John Wiley \& Sons.

40. Bockris, J.M., A.K.N. Reddy, and M. Aldeco, Modern Electrochemistry: Fundamental of Electrodics. 2nd ed. Vol. 2A. 2002, New York: Kluwer Academic Publishers.

41. Hoar, T.P. and D.C. Mears, Corrosion-Resistant Alloys in Chloride Solutions: Materials for Surgical Implants. Proceeding of the Royal Society A, 1966. 294: p. 486-510.

42. Frankel, G.S., Pitting Corrosion of Metals: A Review of the Critical Factors. Journal of the Electrochemical Society, 1998. 145(6): p. 2186-2198.

43. Beck, T.R. and R.C. Alkire, Occurence of Salt Films during Initiation and Growth of Corrosion Pits. Journal of the Electrochemical Society, 1979. 10(126): p. $1662-1666$.

44. U.F.Franck. Electrochemical Studies of Pitting Corrosion of Passive Metals. in 1st International Congress on Metallic Corrosion. 1961. London: Butterworths. 
45. Frankel, G.S., et al., Metastable Pitting of Stainless Steel. Corrosion Science, 1987. 43(7): p. 429-436.

46. Vetter, K.J. and H.H. Strehblow. Pitting Corrosion in an Early Stage and its Theoretical Implications. in NACE 1974. Houston.

47. Chao, C.Y., L.F. Lin, and D.D. Macdonald, A POINT-DEFECT MODEL FOR ANODIC PASSIVE FILMS .1. FILM GROWTH-KINETICS. Journal of the Electrochemical Society, 1981. 128(6): p. 1187-1194.

48. Cooley, L., et al., Annealing to Mitigate Pitting in Electropolished Niobium coupons and SRF Cavities. 2011, Fermilab.

49. Datta, M. and D. Landolt, PIT AND FLOW STREAK FORMATION DURING HIGH-RATE DISSOLUTION OF NICKEL. Journal of the Electrochemical Society, 1982. 129(9): p. 1889-1895.

50. Imboden, R. and R. Sibley, Anodic Polishing of Plain Carbon Steels. Transactions of the Electrochemical Society, 1942. 82: p. 227-239.

51. Madore, C. and D. Landolt, Electrochemical micromachining of controlled topographies on titanium for biological applications. Journal of Micromechanics and Microengineering, 1997. 7: p. 270-275.

52. Palmieri, V., et al. Niobium Electropolishing by Ionic Liquids: What are the naked facts? in SRF 2009. 2009. Berlin, Germany.

53. Shieh, J.M., et al., Reduction of etch pits of electropolished Cu by additives. Journal of the Electrochemical Society, 2004. 151(7): p. C459-C462. 
54. Tajiri, K., et al., Pit-free electropolishing of aluminum and its application for process chamber. Journal of Vacuum Science \& Technology a-Vacuum Surfaces and Films, 1998. 16(3): p. 1196-1200.

55. Van Gils, S., et al., Electropolishing of copper in H3PO4 - Ex situ and in situ optical characterization. Journal of the Electrochemical Society, 2007. 154(3): p. C175-C180.

56. Cao, X. Analysis of Hydrogen Contamination of Superconducting Cavities. in The Fifth Workshop on RF Superconductivity. 1991. DESY, Hamburg, Germany.

57. Higuchi, T. and K.Saito, Hydrogen Absorption in Electropolishing of Niobium. American Institute of Physics Conference Proceedings, 2003. 671(1): p. 203-219.

58. Frankenthal, R., The Effect of Surface Preparation and of deformation on the Pitting and Anodic Dissolution of Fe-Cr Alloys. Corrosion Science, 1968. 8: p. 491-498.

59. $\quad$ http://lepp.cornell.edu/Research/AP/SRF/WebHome.html.

60. Zhao, X., Electropolishing of Niobium in Sulfuric Acid - Methanol Electrolytes: Development of Hydrofluoric Acid-Free Electrolytes, in Materials Science \& Engineering. 2009, Virginia Polytechnic Institute and State University: Blacksburg, Virginia. p. 16.

61. Madore, C., O. Piotrowski, and D. Landolt, Through-Mask Electrochemical Micromachining of Titanium. Journal of the Electrochemical Society, 1999. 146(7): p. 2526-2532. 


\section{Chapter 3 Experimental Procedure}

\subsection{Material and sample preparation}

Niobium samples were saw cut from a $2.8 \mathrm{~mm}$ thick sheet of high purity polycrystalline niobium (99.999\%) of RRR grade used for making SRF cavities. The niobium samples were obtained from Fermi National Accelerator Laboratory (FNAL). The working electrode was used in two configurations. One was a freely exposed niobium surface in a flag electrode configuration of dimension of $1.5 \mathrm{~cm}$ by $0.95 \mathrm{~cm}$ by $0.28 \mathrm{~cm}$ as shown in Fig. 3.1. The other was a masked sample with an exposed area of $1.32 \mathrm{~cm}^{2}$ as shown in Fig. 3.2. The masking was done using a custom built PTFE (Teflon) sample holder with double o-ring fitting to have a good acid seal and prevent crevice. The electrical connection was made using a copper tape to the back side of the niobium and was brought out from the top of the sample holder to avoid contact with the electrolyte as shown in Fig. 3.3. The samples were mechanically polished to 320 grit and then were given a brief electropolishing of 10 minutes for reproducible starting conditions. They

were then cleaned with acetone and isopropyl alcohol. The final cleaning step was ultrasonic cleaning in de-ionized water for 10 minutes. It was then thoroughly dried before exposing them in the electrolytic solution. The counter electrode used was 
$99.999 \%$ aluminum as is the standard practice in the industry for electropolishing of niobium.

\subsection{Electrolyte preparation}

The standard electrolyte used for electropolishing of niobium is a 1:9 volume mixture of HF (48\%) and $\mathrm{H}_{2} \mathrm{SO}_{4}(96.2 \%)$ [1]. $\mathrm{HF}$ and $\mathrm{H}_{2} \mathrm{SO}_{4}$ were obtained from Mallinckrodt Chemicals and Fisher Scientific respectively. The ratio of the acids was varied to investigate the mechanism of electropolishing of niobium. Table 3.1 gives the concentration of fluoride ion and sulfate ion in the solution corresponding to the various ratio of $\mathrm{HF}: \mathrm{H}_{2} \mathrm{SO}_{4}$. The necessary volume of $\mathrm{H}_{2} \mathrm{SO}_{4}$ was added to the appropriate volume of HF because of the higher water content in HF. This was done to avoid overheating and splattering of the acid. The mixing was done in a HDPE container because it is resistant to the highly corrosive mixture. The acid mixture temperature increased during mixing because of the heat of mixing, so the mixture was cooled down to the experimental temperature before starting the experiment by placing it in a water bath with controlled temperature.

\subsection{Experimental setup}

A schematic of the experimental setup is shown in Fig. 3.4. The actual set up is shown in Fig. 3.5. The electrolytic cell was custom made by milling out the inside of a $23 \mathrm{~cm} \mathrm{x}$ $4.8 \mathrm{~cm} \times 5.2 \mathrm{~cm}$ block of $99.999 \%$ pure $\mathrm{Al}$. Al was chosen as the cell material because is passive and thus non-reactive in the corrosive $\mathrm{HF}-\mathrm{H}_{2} \mathrm{SO}_{4}$ mixture. The presence of $\mathrm{HF}$ in the electrolyte ruled out the use of glass and most other materials for use as the cell body. 
Teflon and HDPE are also resistant to HF, but the use of a Teflon cell (which was tried first) prevented good temperature control of the electrolyte because of its low thermal conductivity. The process of electropolishing causes joule heating, so effective heat dissipation through the electrolytic cell body is required to maintain a constant electrolyte temperature. Al has both high thermal conductivity and good resistance to the electrolyte. Al has been commonly used as the counter electrode for $\mathrm{Nb}$ electropolishing [1] so the cell body was also used as the counter electrode in this study. For a uniform current distribution, the sides and bottom of the cell were blocked by thin Teflon sheets. The wall of the cell facing the sample was the only available surface to act as the counter electrode so that a linear current flow was maintained between the working and counter electrodes. The temperature of the electrolyte was maintained within $\pm 0.5^{\circ} \mathrm{C}$ of the intended value by placing the Al cell in a water bath. The water bath temperature was controlled by a coiled copper tube through which water was pumped by a temperature controller. The temperature of the electrolyte was monitored using a thermocouple inserted near the sample.

The reference electrode used for measuring the surface potential was mercury-mercurous sulfate (MSE) reference electrode with a standard potential of $+0.64 \mathrm{~V}$ vs. SHE. MSE reference electrode was used to prevent the problem of chloride contamination associated with the saturated calomel reference electrode. The commercially available MSE reference electrode body is made of glass and so cannot be directly placed in an HF solution. To overcome this problem a bridge tube was made using a medical syringe with a shrink-fitted Teflon tube at the bottom and a Teflon frit was fitted to the end of the 
Teflon tube to maintain ionic conductivity without mixing the contents in the bridge tube with the electrolyte. The MSE reference electrode was placed in the bridge tube which was filled with $0.5 \mathrm{M} \mathrm{H}_{2} \mathrm{SO}_{4}$ to provide an ionic path. The tip of the bridge tube was placed at a distance of about $1 \mathrm{~cm}$ from the $\mathrm{Nb}$ surface to prevent it from blocking the surface.

A potentiostat was not used in these experiments because of the need for large voltages. A computer controlled power supply, Sorensen XDL 35-5P, was used to supply the current between the working electrode and the counter electrode. A Fluke 8845A digital multimeter, which could be interfaced to the computer, was used to measure the surface potential of the niobium working electrode with respect to the MSE reference electrode. The data acquisition was supported by a customized LabVIEW program to control the above electronic equipments. Fig. 3.6 shows the laboratory set up for the electronic equipment and data acquisition system.

\subsection{Electrochemical polarization experiments}

The electrochemical polarization experiments were not performed under potential control because a power supply was used instead of a potentiostat, as has been done by others [2]. In a typical 3 electrode setup, the potentiostat maintains the set surface potential of the working electrode with respect to the reference electrode by passing a sufficient current between the working electrode and the counter electrode [3]. In the present experimental configuration, the power supply applies a potential between the $\mathrm{Nb}$ working electrode and Al counter electrode and reports the current flowing. The 
multimeter measures the potential between the niobium working electrode and the MSE reference electrode.

The electrochemical polarization experiments were carried out by scanning the potential between the working electrode and the counter electrode at a rate of $0.1 \mathrm{~V} / \mathrm{s}$ from $0 \mathrm{~V}$ to $33 \mathrm{~V}$ in most cases. So while the working electrode potential was not carefully controlled, the potential vs. the reference electrode varied with time and the current flow was measured.

\subsection{Constant potential experiments}

Electropolishing was carried out under different conditions by applying a constant voltage between the working electrode and the counter electrode. Current density was measured as a function of time. These experiments were carried out for different times and voltage conditions.

\subsection{Galvanostatic experiments}

The galvanostatic experiments were carried out by applying a constant current between the niobium working electrode and the aluminum counter electrode. The samples were maintained for 200 seconds at open circuit potential before applying a constant current. All the experiments were carried out at a temperature of $26^{0} \pm 0.5^{0} \mathrm{C}$. The potential of $\mathrm{Nb}$ was measured with respect to the MSE reference electrode 


\subsection{Surface characterization}

The surface finish after EP was characterized using optical microscopy, scanning electron microscopy (SEM) and optical profilometry (OP). Atomic Force Microscopy did not give results representative of the entire sample because the scan area is limited to 100 um by $100 \mathrm{um}$. OP, which has a much larger scan area, was used to get the surface topography. The Contour GT K1 OP from Veeco (Fig. 3.7) was used in this work. The supporting software Vision 64 provided RMS surface roughness, $\mathrm{R}_{\mathrm{q}}$, which was used for comparing the surface finish obtained under different conditions The profilometer could scan a maximum area of $1.2 \mathrm{~mm}$ by $0.96 \mathrm{~mm}, 317 \mathrm{um}$ by $238 \mathrm{um}$ and 127 um by $95 \mathrm{um}$ at a magnification of 5X,20X and 50X, respectively. The software also has the feature

of stitching, which makes the scan of huge areas possible. The SEM images were taken using a Quanta 200 with a general purpose tungsten source. 


\subsection{Tables and Figures}

\begin{tabular}{|c|c|c|}
\hline $\begin{array}{c}\text { Volume ratio of } \\
\text { HF: } \mathbf{H}_{2} \mathbf{S O}_{4}\end{array}$ & {$\left[\mathbf{F}^{-}\right] \mathbf{( M )}$} & ${ }^{\left[\mathbf{S O}_{4}{ }^{2-}\right] \mathbf{( M )}}$ \\
\hline $1: 9$ & 2.76 & 16.11 \\
\hline $0.9: 9.1$ & 2.48 & 16.29 \\
\hline $0.8: 9.2$ & 2.21 & 16.65 \\
\hline $0.7: 9.3$ & 1.93 & 16.83 \\
\hline $0.6: 9.4$ & 1.66 & 17.18 \\
\hline $0.4: 9.6$ & 1.10 & 17.54 \\
\hline $0.2: 9.8$ & 0.55 & \\
\hline
\end{tabular}

Table 3.1 Fluoride ion and sulfate ion concentrations corresponding to different volume ratio of $\mathrm{HF}: \mathrm{H}_{2} \mathrm{SO}_{4}$ 


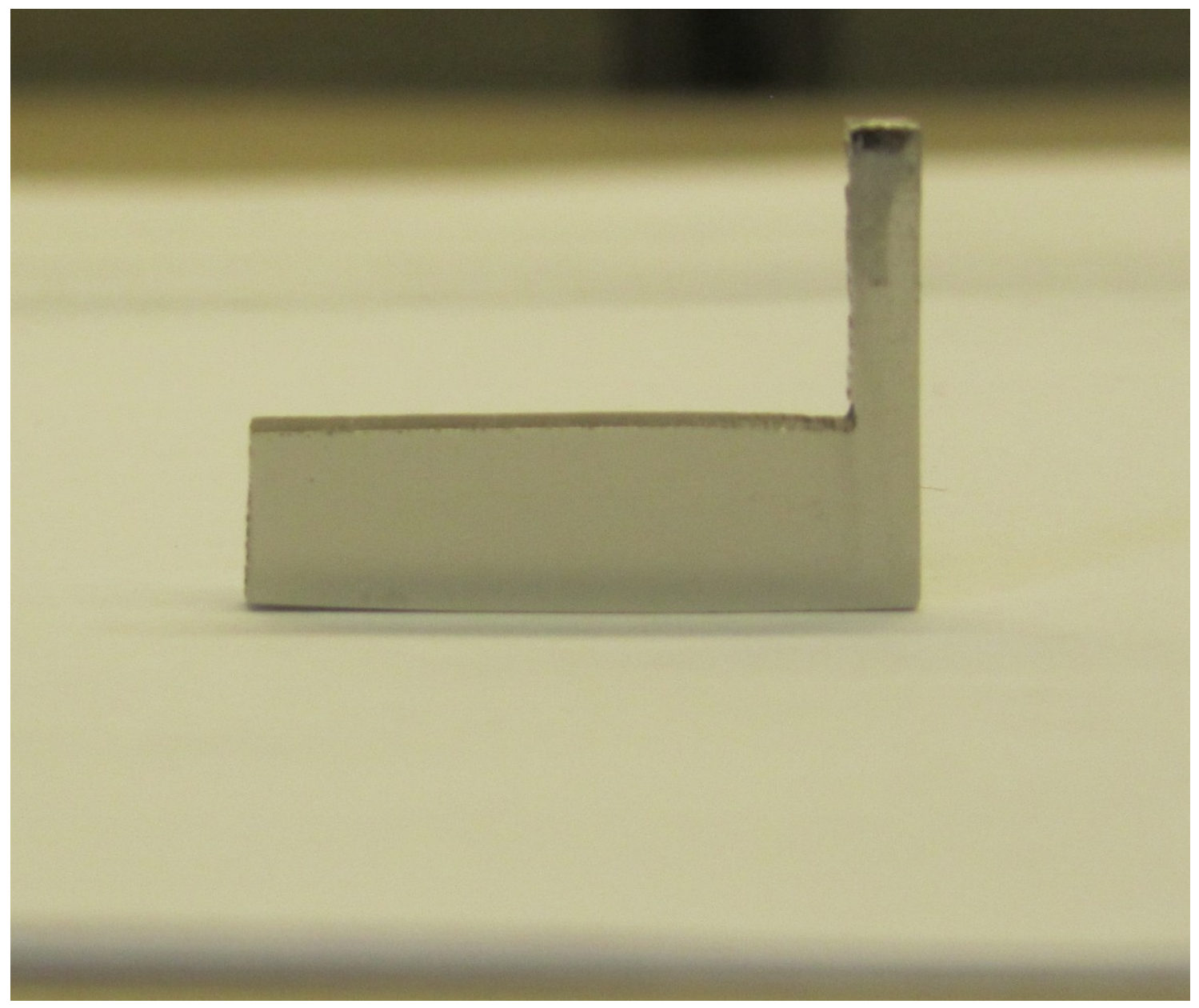

Fig. 3.1: Niobium electrode in flag configuration 


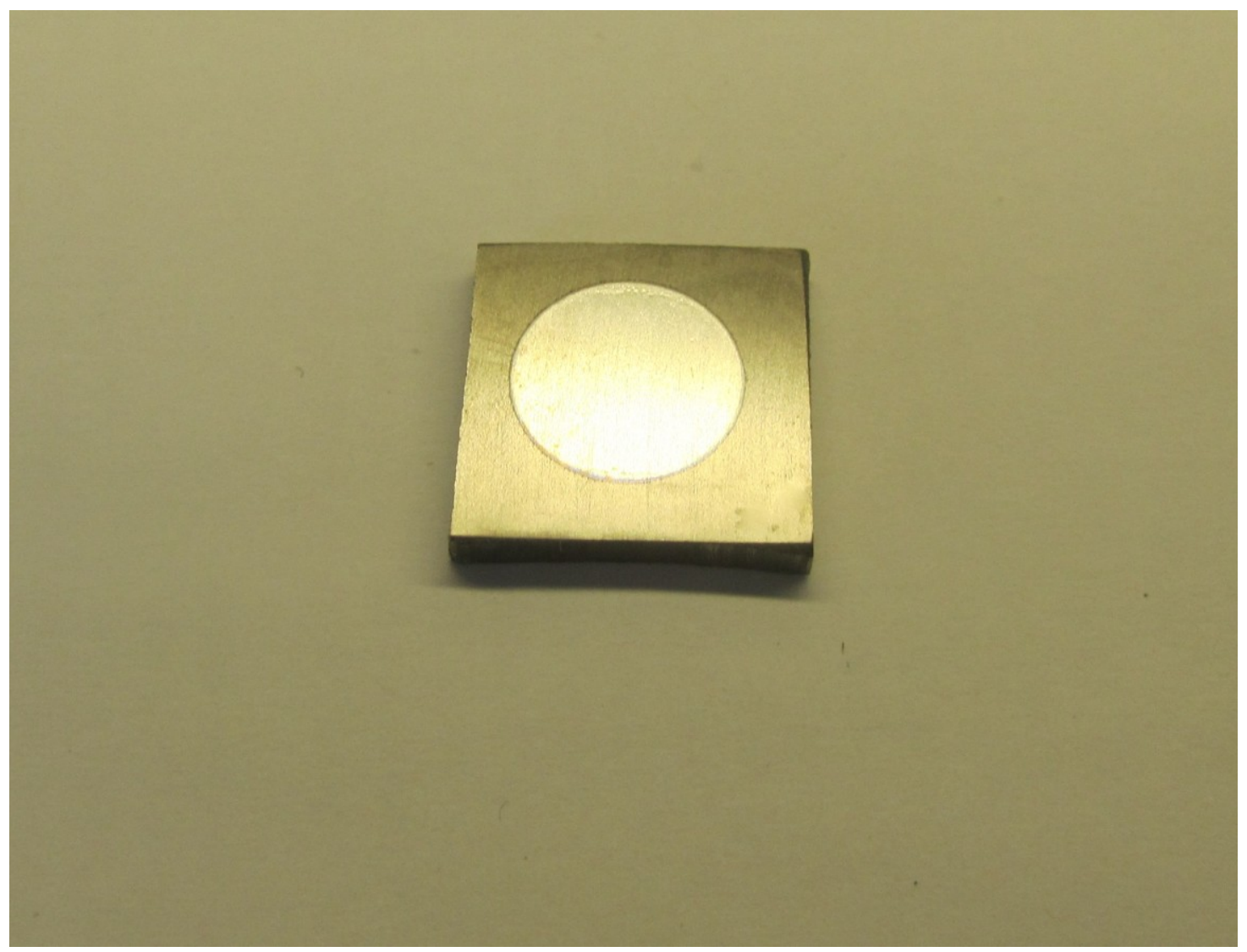

Fig. 3.2: Masked niobium electrode with central circular area exposed. 


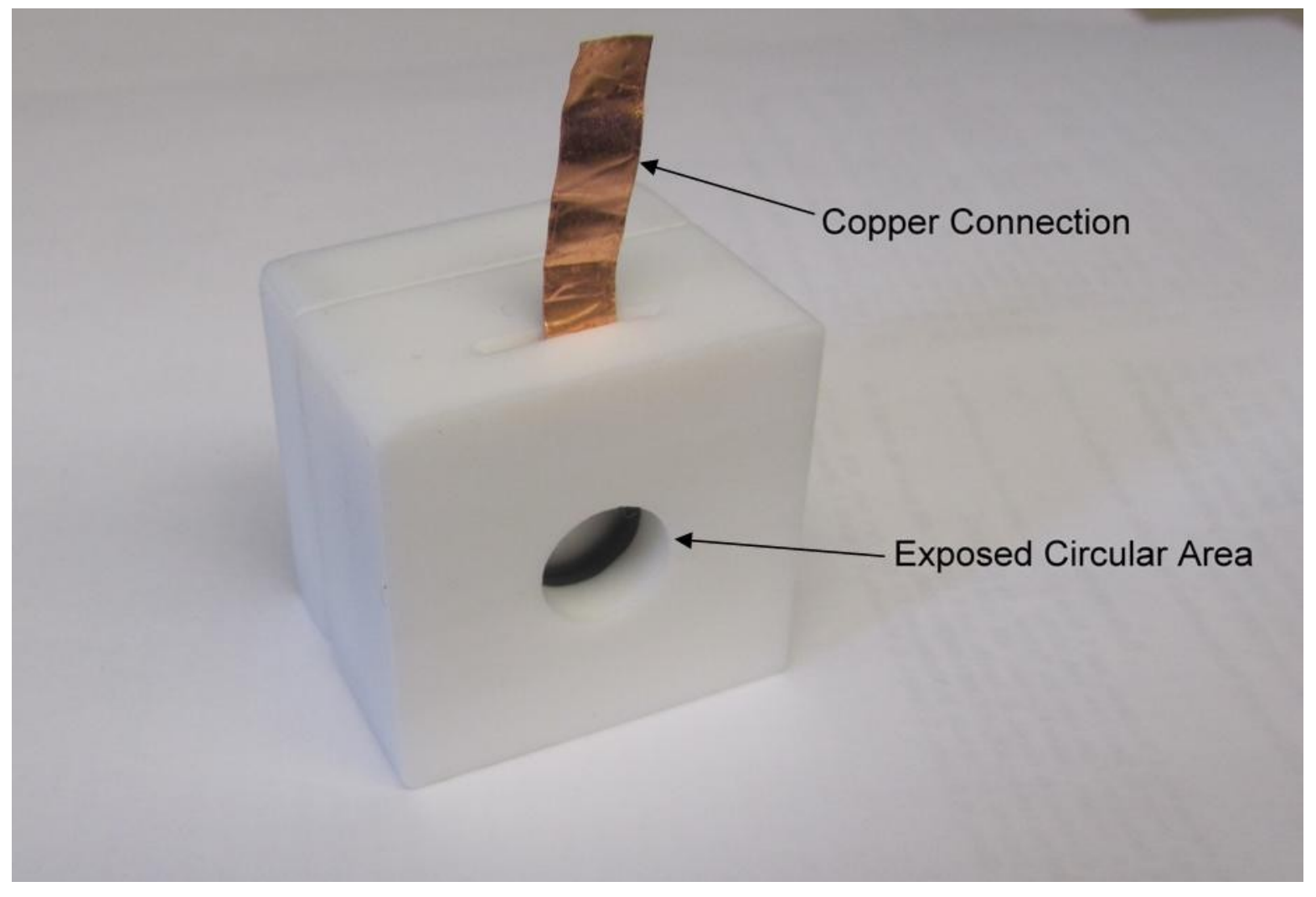

Fig. 3.3: Teflon sample holder for working electrode with an exposed circular central area. 


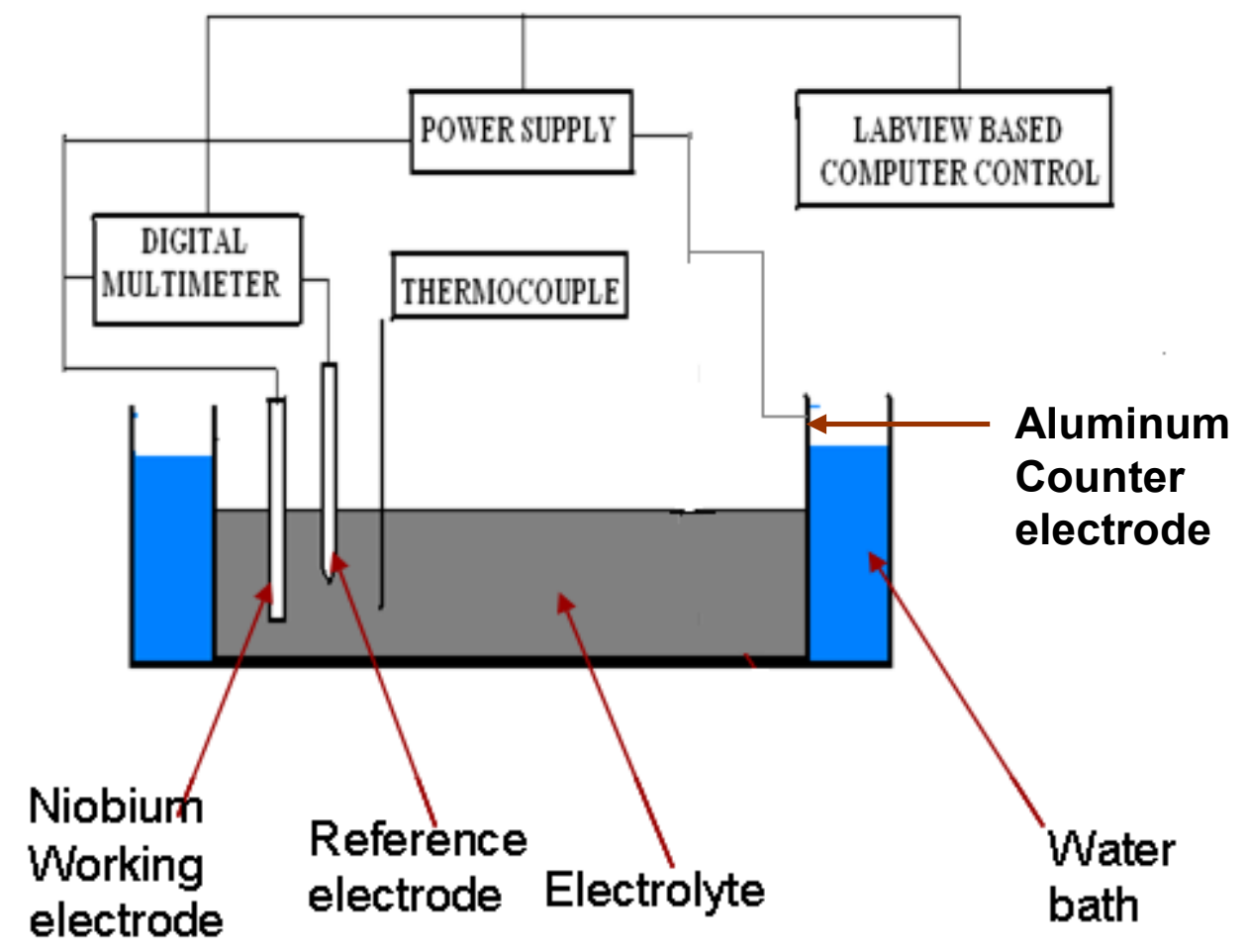

Fig. 3.4: Schematic of the experimental set up for the tests. 


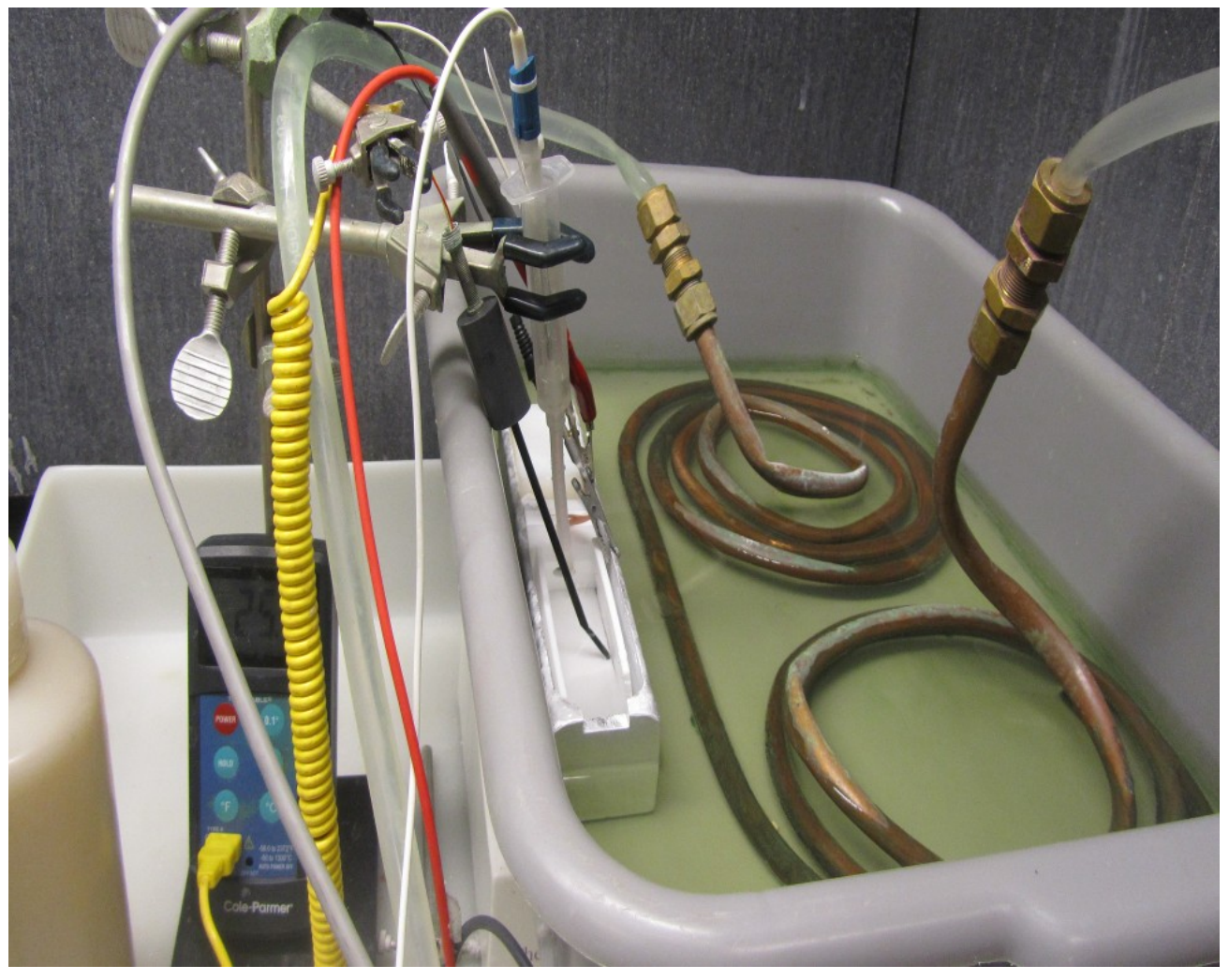

Fig. 3.5: Photograph of the experimental set up. 


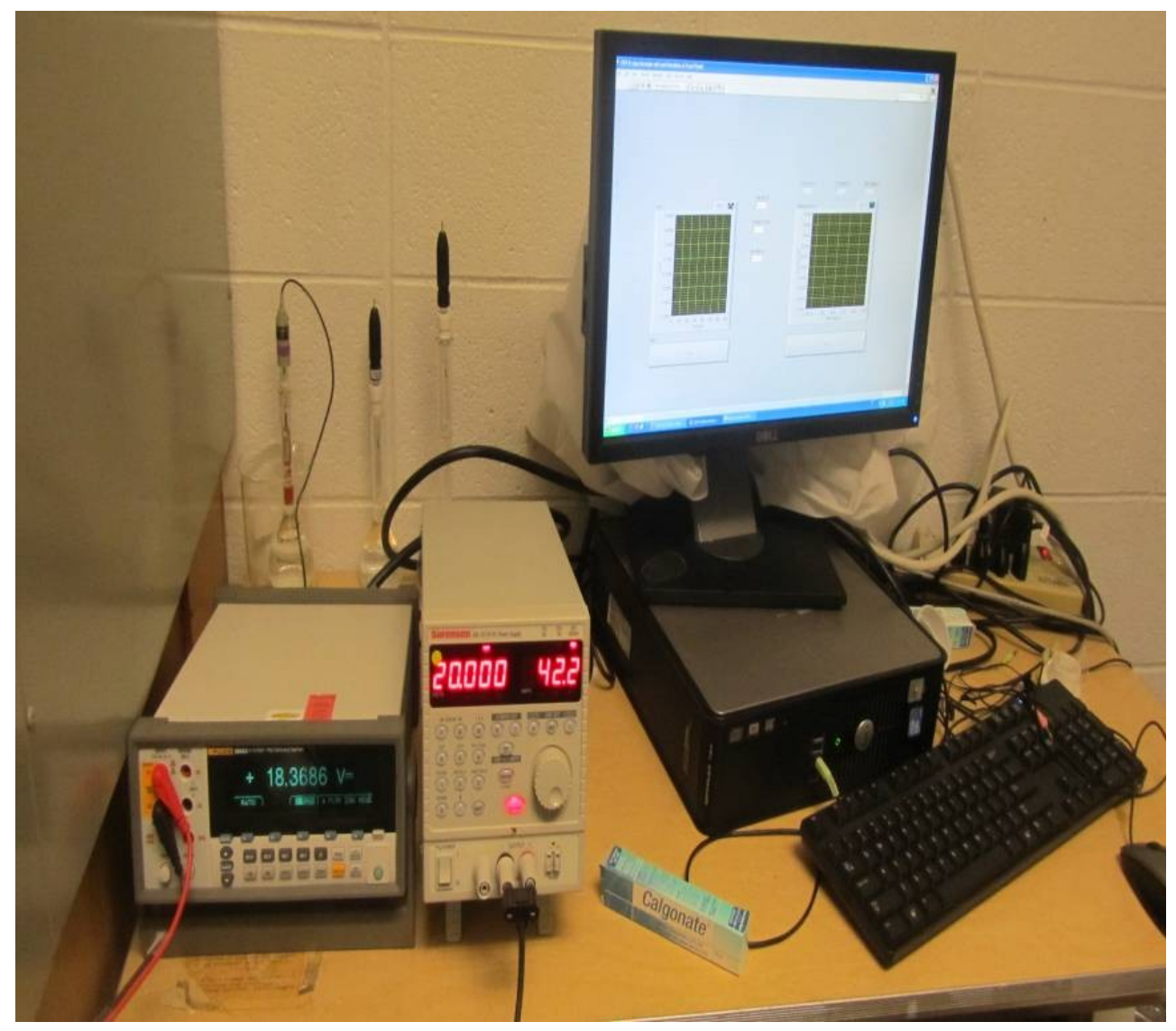

Fig. 3.6: Photograph of the electronic equipments and the data acquisition system. 


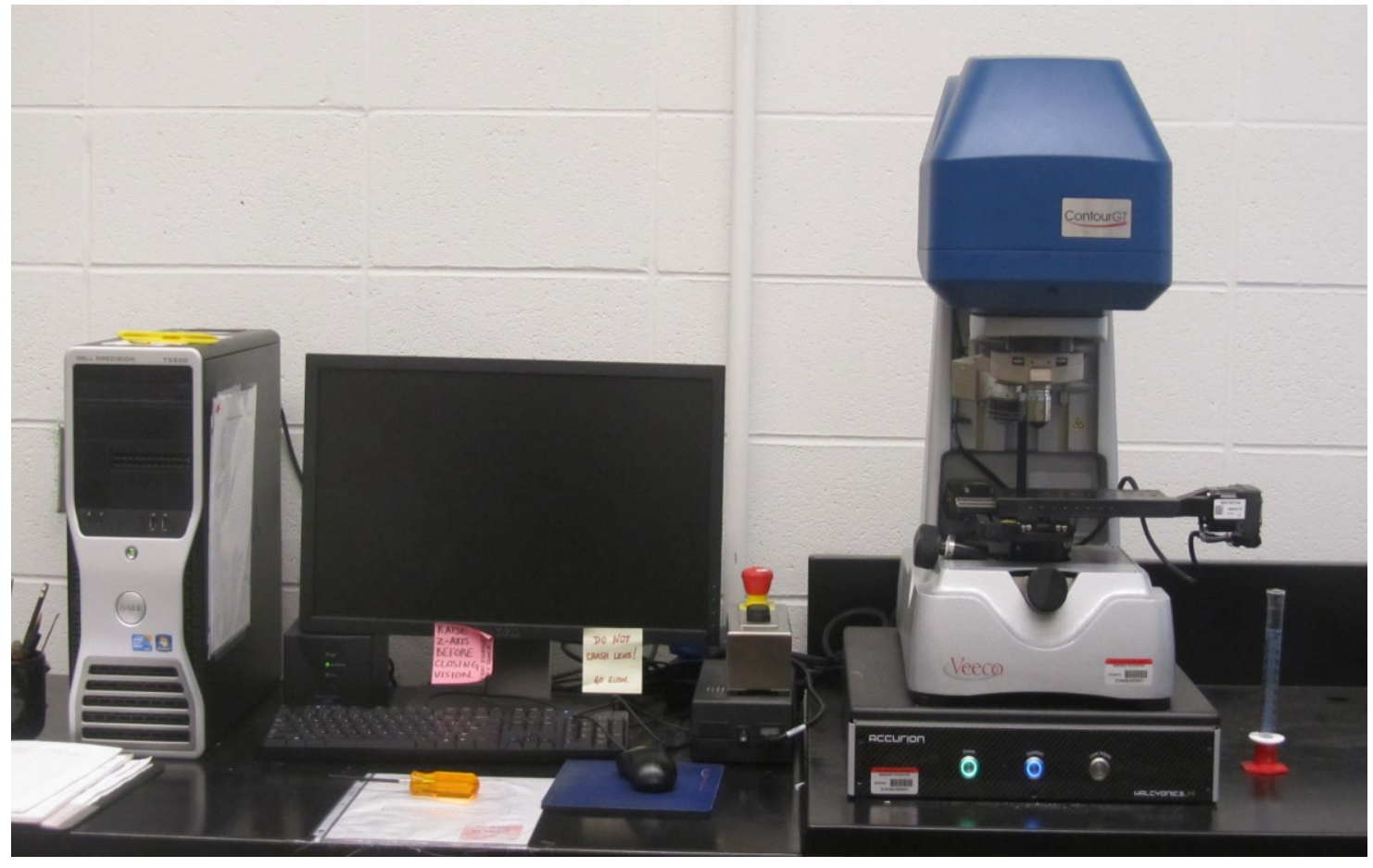

Fig. 3.7: Veeco's contour GT K1 optical profilometer 


\subsection{References}

1. H.Padamsee, RF Superconductivity : Science, Technology, and Applications. 2009, Weinheim: Wiley-VCH.

2. Tian, H., et al., The mechanism of electropolishing of niobium in hydrofluoricsulfuric acid electrolyte. Journal of the Electrochemical Society, 2008. 155(9): p. D563-D568.

3. Landolt, D., Corrosion and Surface Chemistry of Metals. 1 ed. 2007, Lausanne, Switzerland: EPFL Press. 


\section{Chapter 4 Results and Discussion}

The mechanism of niobium electropolishing has been investigated using potentiodynamic polarization experiments and potential transient measurements. The effect of cell voltage and electropolishing time on the surface finish is studied using RMS roughness obtained from surface profile measurements. Visual and experimental evidence highlighting the presence of natural convection is also discussed.

\subsection{General shape of $\mathrm{Nb}$ electrochemical polarization curve}

Fig. 4.1 shows a typical polarization curve measured in this work. It is characterized by an initial exponential increase in current density with anode potential $\left(\mathrm{V}_{\mathrm{a}}\right.$ to $\left.\mathrm{V}_{\mathrm{b}}\right)$, which is due to activation polarization. The current density reaches a peak value and then and often exhibits oscillations. The current density then stabilizes at a plateau value that is relatively constant over a wide range of potential. The current density peak results from super-saturation of the solution and precipitation of a salt film at the electrode surface for systems that exhibit the salt film mechanism of electropolishing [1]. In case of electropolishing systems that follow acceptor mechanism, the salt film does not form. Instead there is a viscous layer close to the electrode surface. The peak at $\mathrm{V}_{\mathrm{b}}$ could be because of a competition between the thickening viscous film and increasing transport rate of anions to the surface, on increasing the applied potential [2].At $\mathrm{V}_{\mathfrak{c}}$, the diffusion 
layer establishes a stable thickness resulting in a constant plateau current density and the system is under mass transport control. It will be shown later that the $\mathrm{Nb}$ electropolishing system follows an acceptor type mechanism

\subsection{Determination of important parameters affecting $\mathrm{Nb}$ electropolishing}

Electrochemical polarization experiments and surface profile measurements after electropolishing were carried out to determine the important parameters affecting the niobium electropolishing system. Measurements of current density (i) vs. surface potential (V) of niobium against the MSE reference electrode were determined by systematically varying the following parameters:

1. Acid ratio of $\mathrm{HF}$ to $\mathrm{H}_{2} \mathrm{SO}_{4}$

2. Temperature

3. Degree of bath agitation

4. Bath age

The effects of the following parameters on surface finish during constant potential measurements were studied by profile measurements:

1. Cell voltage

2. Duration of electropolishing 
The important effects of these parameters on the polarization curve were on the current density plateau magnitude, the voltage range over which the mass transport limitation effect was observed and the surface finish obtained.

\subsubsection{Effect of ratio of $\mathrm{HF}$ to $\mathrm{H}_{2} \mathrm{SO}_{4}$}

The standard electrolyte for electropolishing niobium is 1:9 volume ratio of $\mathrm{HF}: \mathrm{H}_{2} \mathrm{SO}_{4}$ [3]. In this work, the ratio of the acids was varied to study the mechanism. Fig 4.2 shows the effect of differing acid ratio on the polarization curve for niobium electropolishing at a constant temperature of $26^{\circ} \mathrm{C}$. The plot clearly shows, as the fraction of hydrofluoric acid in the solution increases, the limiting current density increases, which is in agreement with the results reported by Tian et al. [4]. This dependence of limiting current density on acid ratio hints at the niobium electropolishing following acceptor ion mechanism with fluoride ion being the transport limited species. This can be inferred from eqn. 1 for limiting current density where the increase in the bulk concentration of acceptor species would result in the increase in the limiting current density.

$$
i_{L}=\frac{n F D C}{\delta}
$$

$\mathrm{n}=$ charge of the ion involved.

$\mathrm{F}=$ Faraday constant

$\mathrm{D}=$ Diffusion coefficient of the rate limiting species. 
$\mathrm{C}=$ saturation concentration of metal ions in the solution for salt film mechanism or bulk concentration of the acceptor species for acceptor mechanism.

$\delta=$ Thickness of the Nernst diffusion layer [5].

Fig. 4.3 shows that the limiting current density value increases linearly with fluoride ion concentration. This supports the notion that fluoride ion is the rate limiting species. This assignment is also supported by the study of potential transients obtained during galvanostatic experiments, as described later.

\subsubsection{Effect of temperature}

Temperature was found to be very important parameter for electropolishing. Fig. 4.4 shows the effect of temperature on the polarization curve for $\mathrm{HF}$ to $\mathrm{H}_{2} \mathrm{SO}_{4}$ ratio of $1: 9$ by volume. The plateau current density increases with increase in temperature, which is in agreement with the observations made by other authors[4]. This result can be related to equation 1 for the limiting current density value. The increase in temperature results in an increase in the value of diffusion coefficient, D of the rate limiting species. The dependence of $\mathrm{D}$ on temperature is given by equation $2[6]$.

$$
D=D_{o} \exp \left(-\frac{Q_{d}}{R T}\right)
$$

where $\mathrm{D}_{\mathrm{o}}=$ Temperature independent pre-exponential

$\mathrm{Q}_{\mathrm{d}}=$ Activation energy for diffusion 
$\mathrm{R}=$ Gas constant

$\mathrm{T}=$ Absolute temperature

The variation of current density with temperature can be obtained by inserting equation 2 in equation 1.

$$
i_{L}=\frac{n F D_{o} C}{\delta} \exp \left(-\frac{Q_{d}}{R T}\right)
$$

Taking natural logarithm of both sides in equation 3 gives equation 4 .

$$
\ln i_{L}=\ln \left(\frac{n F D_{o} C}{\delta}\right)-\frac{Q_{D}}{R T}
$$

Fig 4.5 shows a plot of $\ln \mathrm{i}_{\mathrm{L}} \mathrm{vs} . \mathrm{T}^{-1}$. Analysis of the plot gives a value of $33 \mathrm{~kJ} / \mathrm{mole}$ for $\mathrm{Q}_{\mathrm{d}}$ and the value of $\mathrm{D}_{\mathrm{o}}$ as $2.24 \times 10^{-6} \mathrm{~m}^{2} / \mathrm{s}$ assuming $\delta=20 \mu \mathrm{m}$, as estimated by Tian et al. [7].

\subsubsection{Effect of convection}

Stirring of the electrolyte during electropolishing of niobium is an important parameter to consider given that the industry practice employed for the processing of SRF cavities involves rotating partially filled cavities [3]. Fig. 4.6 shows stirring leads to an increase in the limiting current density for a niobium electrode in the standard electrolyte composition at $22^{\circ} \mathrm{C}$. This can again be related to equation 1 through the magnitude of the Nernst diffusion layer thickness, $\delta$. Stirring decreases $\delta$ and thus increases $i_{L}$. This 
indicates that electrolyte agitation should be controlled to a level such that the rate of polishing is not too high and the electrolyte flow continues to be laminar.

It has been shown by Tian et al. that the temperature of the electrolyte near the $\mathrm{Nb}$ electrode is slightly higher than the bulk of the electrolyte [4]. Stirring would result in removal of excess heat, resulting in the surface temperature being closer to the bulk temperature. This lower surface temperature would decrease limiting current density. However, the decrease in diffusion layer thickness on stirring more than offsets the effect of lower surface temperature on the limiting current density and there is an overall increase in $\mathrm{i}_{\mathrm{L}}$ on stirring.

\subsubsection{Effect of bath age}

It is a standard practice in industry to reuse the electrolyte to keep the costs low as well as to generate less hazardous waste acid [3]. Fig. 4.7 shows that the limiting current density value at $30^{\circ} \mathrm{C}$ is lower for an electrolyte that was previously used for $5 \mathrm{~h}$ of electropolishing, compared to fresh electrolyte. This decrease occurs because the ions involved in electropolishing get used up during use. The fluoride ions are consumed in forming one or more of the following soluble species $-\mathrm{H}_{2} \mathrm{NbOF}_{5}, \mathrm{HNbF}_{6}, \mathrm{NbF}_{5}$,

$\mathrm{H}_{6} \mathrm{NbO}_{2} \mathrm{~F}_{7}, \mathrm{H}_{2} \mathrm{NbF}_{7}, \mathrm{NbF}_{3}\left(\mathrm{SO}_{3} \mathrm{~F}\right)_{2} .2 \mathrm{H}_{2} \mathrm{O}, \mathrm{Nb}_{2} \mathrm{O}\left(\mathrm{SO}_{4}\right)_{4}, \mathrm{~S}_{2} \mathrm{O}_{5} \mathrm{~F}_{2}$ [8]. Fluoride ion could also decrease in the electrolytic solution as a result of evaporation of HF as it has a low vapor pressure [3]. The changes shown in Fig. 4.7 indicate that the control of bath chemistry is important. 


\subsubsection{Effect of cell voltage}

The voltage applied between the working electrode and the counter electrode during electropolishing should put the system in the current plateau domain of the obtained current density vs. cell voltage polarization curve to obtain a good surface finish. However, the voltage value on the current plateau used for electropolishing also has a profound effect on the degree of surface finish. Fig. 4.8 shows optical profilometry images obtained for niobium that was electropolished in the standard electrolytic mixture at $26^{\circ} \mathrm{C}$ for 1 hour at different cell voltages along the current density plateau. The values of the corresponding surface potential and RMS roughness $\left(\mathrm{R}_{\mathrm{q}}\right)$ are given in Table 4.1 . The results clearly indicate that $\mathrm{R}_{\mathrm{q}}$ decreases, and thus the surface finish improves, as the polishing voltage is increased, which is in agreement with reports in the literature [2].

\subsubsection{Effect of duration of electropolishing}

The tradeoff of electropolishing time and surface finish must be understood because the cost of processing the SRF cavities will increase as the electropolishing time increases. At minimum, a damaged layer $100-150 \mu \mathrm{m}$ in thickness has to be removed from the niobium surface to result in a surface that is good enough to achieve maximum accelerating gradients [9]. The surfaces of samples electropolished for $0,2,4$ or 6 hours were characterized by optical profilometry over a scan area of $238 \mu \mathrm{m}$ by $318 \mu \mathrm{m}$ as shown in Fig. 4.9. The standard electrolyte was used, the temperature was controlled at $26^{\circ} \mathrm{C}$ and a cell voltage of $15 \mathrm{~V}$ was applied. Table 4.2 lists $\mathrm{R}_{\mathrm{q}}$ values and the depth of material removed after different durations of electropolishing. The depth of material 
removed, 1 , was calculated from the average current density over the polishing period, i, and the polishing time, t, using Faraday's law.

$$
l=\frac{i M t}{n F \rho}
$$

where

$$
\begin{aligned}
& \mathrm{F}=\text { Faraday constant }=96487 \mathrm{C} / \mathrm{equiv} \\
& \mathrm{M}=\text { Atomic } \text { weight of niobium }=92.9 \mathrm{~g} / \mathrm{mol} \\
& \mathrm{n}=\text { valence }=5 \mathrm{eq} / \mathrm{mol} \\
& \rho=\text { density of niobium }=8.57 \mathrm{~g} / \mathrm{cm}^{3}
\end{aligned}
$$

The surface topography changes from a scalloped appearance after $2 \mathrm{~h}$ of electropolishing to a smoother surface after $6 \mathrm{~h}$. The $\mathrm{R}_{\mathrm{q}}$ value improved from $415 \mathrm{~nm}$ for $2 \mathrm{~h}$ of electropolishing to $241 \mathrm{~nm}$ for $4 \mathrm{~h}$ of electropolishing, which is a decrease of $174 \mathrm{~nm}$. After $6 \mathrm{~h}, \mathrm{R}_{\mathrm{q}}$ decreases $73 \mathrm{~nm}$ further to $168 \mathrm{~nm}$. This suggests that the rate of improvement of surface finish decreases with electropolishing time. The required roughness and depth of material to be removed for optimum performance must be considered to minimize the duration of electropolishing. 


\subsection{Galvanostatic Experiments}

Galvanostatic experiments give insights to help to confirm the mechanism of niobium electropolishing. Specifically, systematic variation of applied current density and acid concentration confirm that fluoride ion is the mass transport limiting species.

Application of a current density at or above the limiting current density plateau results in a potential transient with specific characteristics as shown in Fig. 4.10. Four distinct regions can be identified on the curve. There is an instantaneous initial jump of about 0.9 $\mathrm{V}$ on switching on the current, shown as $\mathrm{AB}$ in the figure. This can be attributed to the ohmic potential drop associated with the electrolyte resistance between the reference and working electrodes and then rapid charging of the double layer capacitance. The potential then slowly increases in the region labelled as BD. The overpotential BD has contributions from both activation and concentration overpotential. However, the slow increase along $\mathrm{BD}$ is only due to concentration overpotential. The concentration overpotential arises because the interfacial concentration of the reacting ion is not the same as in the bulk and it changes with time [10].After a certain period of time, a very large jump in potential is observed, labelled DE. The measured potential levels off at around $33 \mathrm{~V}$ MSE, but this is an experimental artifact caused by the limited compliance of the power supply. The current was switched off at point $\mathrm{F}$ which led to the precipitous fall of the potential to the OCP value at G. The value of the potential before the large jump in current is in the activation region of the polarization curve $\left(\mathrm{V}_{\mathrm{a}}\right.$ to $\mathrm{V}_{\mathrm{b}}$ region of Fig. 4.1). 
A potential transient for the application of current below the limiting current density value is shown in Fig. 4.11. The first part of the response is identical to that for the limiting current density case. The potential increases instantaneously, and then slowly increases further to a steady state value. However, below the limiting current density, the very large potential spike is not observed. This can be explained on the basis of the analysis to follow.

The current immediately after being switched on is largely used for charging of the double layer. This is followed by dissolution under mixed control. The overpotential can be described as a sum of activation polarization and concentration overpotential. Based on the potentiodynamic experiments described above, it can be assumed that the rate limiting step is the transport of the acceptor ion fluoride ion to the niobium electrode surface. Assuming that the niobium electro-dissolution proceeds according to the reaction,

$$
\mathrm{Nb}+5 \mathrm{~F}^{-} \rightarrow \mathrm{NbF}_{5}+5 \mathrm{e}^{-}
$$

the surface potential of niobium can be given according the following relation [1]:

$$
E=E_{r e v}+\frac{R T}{\beta n F} \ln \left(\frac{i}{i_{0}}\right)-\frac{R T}{\beta n F} \ln \left(1-\frac{i}{i_{\text {lim }}}\right) .
$$

Where $E_{\text {rev }}$ is the reversible or equilibrium potential and $i_{0}$ represents the exchange current density of the reaction given above. 
The dissolution current associated with the diffusion flux can be given according to relation 7 .

$$
i=\frac{n F D\left(\left[F^{-}\right]_{\text {bulk }}-\left[F^{-}\right]_{\text {surface }}\right)}{\delta}
$$

The limiting current density, $i_{\text {lim }}$ would be obtained when the surface concentration of fluoride ion goes to zero and is given by relation 8 .

$$
i_{\lim }=\frac{n F D\left[F^{-}\right]_{b u l k}}{\delta}
$$

Plugging in equation 7 and 8 in equation 6 gives the variation of surface potential as

$$
E=E_{\text {rev }}+\frac{R T}{\beta n F} \ln \left(\frac{i}{i_{0}}\right)-\frac{R T}{\beta n F} \ln \left(\frac{\left[F^{-}\right]_{\text {surface }}}{\left[F^{-}\right]_{\text {bulk }}}\right)
$$

The variation of fluoride ion concentration with distance, $\mathrm{x}$, and time, $\mathrm{t}$, is given by equation 10, which has been derived based on a semi-infinite one dimensional diffusion model [11].

$$
\left[F^{-}\right]_{x, t}=\left[F^{-}\right]_{b u l k}-2 J_{D} \frac{\sqrt{t}}{\sqrt{\pi D}} \exp \left(-\frac{x^{2}}{4 D t}\right)+\frac{J_{D} x}{D} \operatorname{erfc}\left(\frac{x}{\sqrt{4 D t}}\right)
$$

where $J_{D}$ is the flux due to diffusion and $\mathrm{D}$ is the diffusion coefficient of the fluoride ions. 
The concentration of the fluoride ion at the niobium electrode surface at any time, $t$ is given by equation 11 , which is obtained by substituting $\mathrm{x}=0$ in equation 10 .

$$
\left[F^{-}\right]_{\text {surface, }}=\left[F^{-}\right]_{\text {bulk }}-2 J_{D} \frac{\sqrt{t}}{\sqrt{\pi D}}
$$

The diffusional flux is related to the applied current density by equation 12 .

$$
J_{D}=\frac{i}{n F}
$$

Substituting equation 12 in equation 11 gives the variation of fluoride ion concentration at the niobium electrode surface with time and applied current density, i.

$$
\left[F^{-}\right]_{\text {surface, }}=\left[F^{-}\right]_{\text {bulk }}-2 \frac{i}{n F} \frac{\sqrt{t}}{\sqrt{\pi D}}
$$

Plugging equation 13 in equation 9 gives the variation of surface potential of niobium with time:

$$
E=E_{\text {rev }}+\frac{R T}{\beta n F} \ln \left(\frac{i}{i_{0}}\right)-\frac{R T}{\beta n F} \ln \left(\frac{\left[F^{-}\right]_{b u l k}-2 \frac{i}{n F} \frac{\sqrt{t}}{\sqrt{\pi D}}}{\left[F^{-}\right]_{b u l k}}\right)
$$

The concentration of fluoride ion at the surface decreases with time and ultimately falls to zero, thus making the natural logarithmic term in equation 14 go to negative infinity. This 
would result in the surface potential term increasing toward positive infinity, which is observed for current densities at or above the limiting current density value (Fig. 4.10). The potential transient (Fig. 4.11) for the case of applied current below the limiting current density show no such potential spike towards infinity. This indicates that the surface concentration for fluoride ion does not fall to zero when the applied current density is below the limiting current density value.

A plot of the variation of concentration polarization (the third term in equation 14) with time is shown in Fig. 4.12 for the standard electrolyte composition. It is similar to the variation of surface potential as shown in the inset graph of Fig. 14.10. There is an offset of about $0.4 \mathrm{~V}$ which can be accounted for in the first two terms of equation 14.

Interestingly, the limiting current plateau region observed during potentiodynamic polarization is often considered to be associated with diffusion controlled dissolution. However, the surface concentration of the diffusion limited species cannot be equal to zero or the potential would be infinite as was found for the galvanostatic experiments. The surface concentration in this region under potential control is likely "near zero" as described by Matlosz [12], but not equal to zero.

The results indicate that the niobium electropolishing mechanism is an acceptor mechanism. The overpotential that develops in the salt film mechanism is because of ohmic drop across the salt film, which thickens over the current density plateau in the polarization curve. A $33 \mathrm{~V}$ drop in potential cannot be expected across a salt film, which rules out the salt film mechanism. The potential jump can be explained coherently with 
the logarithmic term in equation 9 going to negative infinity with the fluoride ion concentration at the surface going to zero, thus confirming that the niobium electropolishing mechanism is associated with a diffusion limited transport of fluoride ions to the electrode surface.

Tian et al. studied the impedance response of $\mathrm{Nb}$ electropolishing system and proposed that a compact salt or oxide film exists along the current density plateau and the dissolution rate is determined by the diffusion limited approach of fluoride ions to the $\mathrm{Nb}$ surface [4]. The potential transient study done in this work confirms that the electropolishing rate is governed by the diffusion limitation of fluoride ions. However, there is no evidence for the formation of a salt film.

An important figure of merit is the transition time $(\tau)$, the time required for the concentration of fluoride ion at the surface to go to zero. $\tau$ is defined as the time from point $A$ when the current was switched on to point $\mathrm{D}$ when the potential spike took place in Fig. 4.10. $\tau$ can be obtained by setting $\left[\mathrm{F}^{-}\right]_{\text {surface, }}$ in equation 13 to zero, thus giving equation 15

$$
\tau=\frac{n^{2} F^{2} \pi D}{4 i^{2}}\left[F^{-}\right]_{b u l k}^{2}
$$

Equation 15 is a form of Sand's equation [13]. The value of $\tau$ was determined for different values of applied current density, $\mathrm{i}$ and $\left[\mathrm{F}^{-}\right]_{\text {bulk. }}$. The ratio of $\mathrm{HF}$ to $\mathrm{H}_{2} \mathrm{SO}_{4}$ was varied from a ratio of 1:9 to $0.6: 9.4$, so the concentrations of both fluoride ion and sulfate 
ion were changed. Fig. 4.13 shows that for a given acid ratio, $\tau$ increases with decreasing applied current density, i. In fact, a linear relationship between $\tau$ and $\mathrm{i}^{-2}$ is observed for a given acid ratio and values of $i$ greater than the limiting current density (Fig. 4.14), as predicted by the Sand's equation. This supports the idea that the jump in potential is associated with concentration polarization occurring with acceptor ion diffusion limitation. However, the straight line does not pass through the origin as predicted by equation 15. Fig. 4.15 shows that $\tau$ increases as the fluoride ion concentration increases. There is a linear dependence of $\tau$ on the square of the fluoride ion concentration, which is again in accordance with Sand's equation and confirms that diffusion of the fluoride ion is rate limiting at the current density plateau in the potentiodynamic curve. However, this curve also does not pass through the origin as dictated by the Sand's equation. The slope of the curves in Fig. 4.14 and Fig. 4.15 gives diffusion coefficient of fluoride ion in the order of $10^{-11} \mathrm{~m}^{2} / \mathrm{s}$. A lower slope for the time vs. $\mathrm{i}^{-2}$ curve (Fig. 4.14) would have resulted in the curve passing through the origin. The observed higher slope of the $\tau$ vs $i^{-2}$ curve implies that the measured transient time is of a lower value than would be expected for a system perfectly obeying a semi-infinite one dimensional diffusion model. Transient time calculated for a semi-infinite one dimensional diffusion model (Fig. 4.13) does give values which are larger than those obtained experimentally. For example, the calculated and measured times were approximately $200 \mathrm{~s}$ and $120 \mathrm{~s}$, respectively, for an applied current density of $25.75 \mathrm{~mA} / \mathrm{cm}^{2}$ in the standard electrolyte mixture. Similarly, at $27.27 \mathrm{~mA} / \mathrm{cm}^{2}$ the calculated and measured transient times were approximately $90 \mathrm{~s}$ and $170 \mathrm{~s}$, respectively. The smaller measured transient time could be interpreted as evidence 
of an increased rate of transport. As described presently, this behavior could be due to a higher effective diffusion coefficient of fluoride ions or to effects of migration.

One reason for the smaller measured transition times and the lines not passing through the origin could be higher temperature in the vicinity of the electrode, which would result in an increase in diffusion coefficient locally as given by equation 2 . This explanation seems viable owing to the fact that the electropolishing system generated heat, which was evident by a temperature increase of the electrolyte if the temperature was not controlled by an external water bath. Despite the good control of the bulk temperature in this experimental setup, the temperature near the electrode and thus the diffusivity were probably higher than bulk values.

Another possible reason for increased transport rate could be migration affecting the transport process. Kuo and Landolt studied the anodic dissolution of iron in concentrated chloride media using a rotating disc electrode [14]. The concentration of the dissolving iron chloride at the electrode surface obtained using Levich equation gave a value higher than the saturation concentration of $\mathrm{FeCl}_{2}$ in the electrolyte. This was explained on the basis of influence of migration in increasing the rate of transport of iron ions away from the electrode surface to the bulk electrolyte. The authors defined an empirical "effective" diffusion coefficient of a magnitude higher than the ionic diffusion coefficient of iron ions to account for the observed experimental deviations from ideality. Similarly, the higher slope obtained for the $\tau$ vs. $\mathrm{i}^{-2}$ could be explained based on a higher "effective" diffusion coefficient of fluoride ion associated with migration. 
Natural convection could also be playing a role in the transport process of the ions to and from the electrode surface. There was visual and experimental evidence to the presence of this effect, which will be discussed in the next section.

\subsection{Role of convection in electropolishing of niobium}

The results of the galvanostatic experiments suggested that transport occurring during electropolishing of niobium might not be purely diffusional and can be affected by other transport processes like migration and convection. Visual observations along with some electrochemical experimental results point to the effects of convection on the transport process.

A bluish-green layer could be seen to slowly flow down a vertically oriented niobium electrode at the end of electropolishing when the applied power was terminated. Such a layer has also been observed by Eozenou et al [8]. It has not been possible to analyze the nature of this layer by ex-situ methods as it vanishes as soon as the current is switched off. The exact composition of the reaction products formed on electro-dissolution is still unknown because of this limitation. There is a higher concentration of the dissolved products $\left(\mathrm{H}_{2} \mathrm{NbOF}_{5}, \mathrm{HNbF}_{6}, \mathrm{NbF}_{5}, \mathrm{H}_{6} \mathrm{NbO}_{2} \mathrm{~F}_{7}, \mathrm{H}_{2} \mathrm{NbF}_{7}, \mathrm{NbF}_{3}\left(\mathrm{SO}_{3} \mathrm{~F}\right)_{2} .2 \mathrm{H}_{2} \mathrm{O}\right.$, $\left.\mathrm{Nb}_{2} \mathrm{O}\left(\mathrm{SO}_{4}\right)_{4}, \mathrm{~S}_{2} \mathrm{O}_{5} \mathrm{~F}_{2}\right)[8]$ in the immediate vicinity of the electrode surface resulting in a higher density of the electrolyte in this region. The distinct color observed can be due to the color of the dissolved compounds which are present in high concentrations near the $\mathrm{Nb}$ electrode surface. The sliding down of the viscous layer can also be considered as an indication of the presence of natural convection in the system. 
There is other evidence that natural convection plays a role during electropolishing of niobium. Fig. 4.16a shows a sample that was electropolished in the exposed circular area in a vertical configuration. Fig. 4.16b shows a plot of depth of niobium removed in $\mu \mathrm{m}$ as a function of distance from point A to point B marked in Fig. 4.16a. The depth of niobium removed was determined by optical profilometry. The plot shows that more material was removed from the top of the circle than from the bottom. The removal depth varied almost linearly along most of the surface until reaching a constant value that was at a depth of approximately $17 \mu \mathrm{m}$ less than at the top part of the vertical sample. This clearly suggests that natural convection played a role. The diffusion layer thickness was smaller at the top of the sample compared to the bottom, resulting in a higher dissolution rate at the top of the sample. The recess of the sample in the sample holder likely resulted in less of free convection at the bottom of the sample.

Fig. 4.17 shows the optical micrograph of the front surface of a niobium electrode that was electropolished in the flag electrode configuration. Ridges were observed in the direction of gravity at the edge of the sample, which is also likely related to the downward flow of the viscous layer under the effect of gravity. The result clearly points to the presence of natural convection which is schematically shown in Fig 4.18.

Fig. 4.19 shows an image of the bottom surface of the same flag electrode that was shown in Fig. 4.17. Extensive pitting is evident on the bottom surface, which was facing down in the electrolyte while the front surface shows no such pitting. The formation of heavy dissolution products at the bottom surface under the effect of gravity could have created 
turbulent flow rather than the laminar flow that likely occurred on the vertical front face. Such turbulence would disrupt the boundary layer, result in a form of localized attack that looks like pitting. However, the mechanism is very different than pitting corrosion observed in passive materials exposed to aggressive solutions such as chloride solutions. This pitting does not result from the localized breakdown of a passive film, but rather from convection in the form of local eddy currents that stabilize in the downward facing condition. This indicates the importance of a stable diffusion regime for the electropolishing process to take place. This result is very important keeping in mind the fact that the SRF cavities are rotated during electropolishing. Such rotation could result in turbulence that leads to pitting, which would severely affect the performance of these cavities if the rotation speed is not properly optimized.

It is clear from the above results that hydrodynamics and gravity conditions play a great role in the electropolishing of niobium. Due to the complex shape of the SRF cavities and hence the varying hydrodynamic and gravity conditions, the diffusion layer thickness may vary over the contour of the cavities. This results in a varying removal rate. Fig. 4.20 compares the effect of orientation on the electropolishing current density and hence the removal rate. Two masked electrodes were electropolished for two hours each under identical conditions, one with its surface oriented vertically and the other with its surface horizontal, facing up in the electrolyte. The constant current density obtained is due to diffusion limited access of fluoride ions to the surface with the fluoride ion concentration at the surface dropping to near zero concentration. The diffusion layer formed on the surface of the electrode with face up configuration was thicker than the one formed on 
the vertical electrode for the same experimental parameters. As a result, the current density for the face up configuration was lower than that of vertical electrode configuration. This comparison points to the importance of the role of hydrodynamics in electropolishing. The hydrodynamic conditions prevailing at the surface of the face up electrode is much different from that prevailing at the vertical electrode surface. 


\begin{tabular}{|l|c|c|c|c|}
\hline Polishing Cell Voltage (V) & 5 & 10 & 15 & 20 \\
\hline Nb Surface Potential (V) & 2.9 & 8.1 & 12.7 & 16.7 \\
\hline Rq (um) & 1.135 & .787 & .615 & .431 \\
\hline
\end{tabular}

Table 4.1: Effect of Polishing voltage on RMS roughness for 1 hour electropolishing at $26^{\circ} \mathrm{C}$ in standard electrolyte.

\begin{tabular}{|c|c|c|}
\hline Polishing time (h) & Rq (nm) & Thickness removed (um) \\
\hline 0 & 504 & 0 \\
\hline 2 & 415 & 50.1 \\
\hline 4 & 241 & 108.6 \\
\hline 6 & 168 & 156.8 \\
\hline
\end{tabular}

Table 4.2: Effect of electropolishing duration on RMS roughness and niobium thickness removed for electropolishing at a cell voltage of $15 \mathrm{~V}$ at $26^{\circ} \mathrm{C}$ in standard electrolyte. 


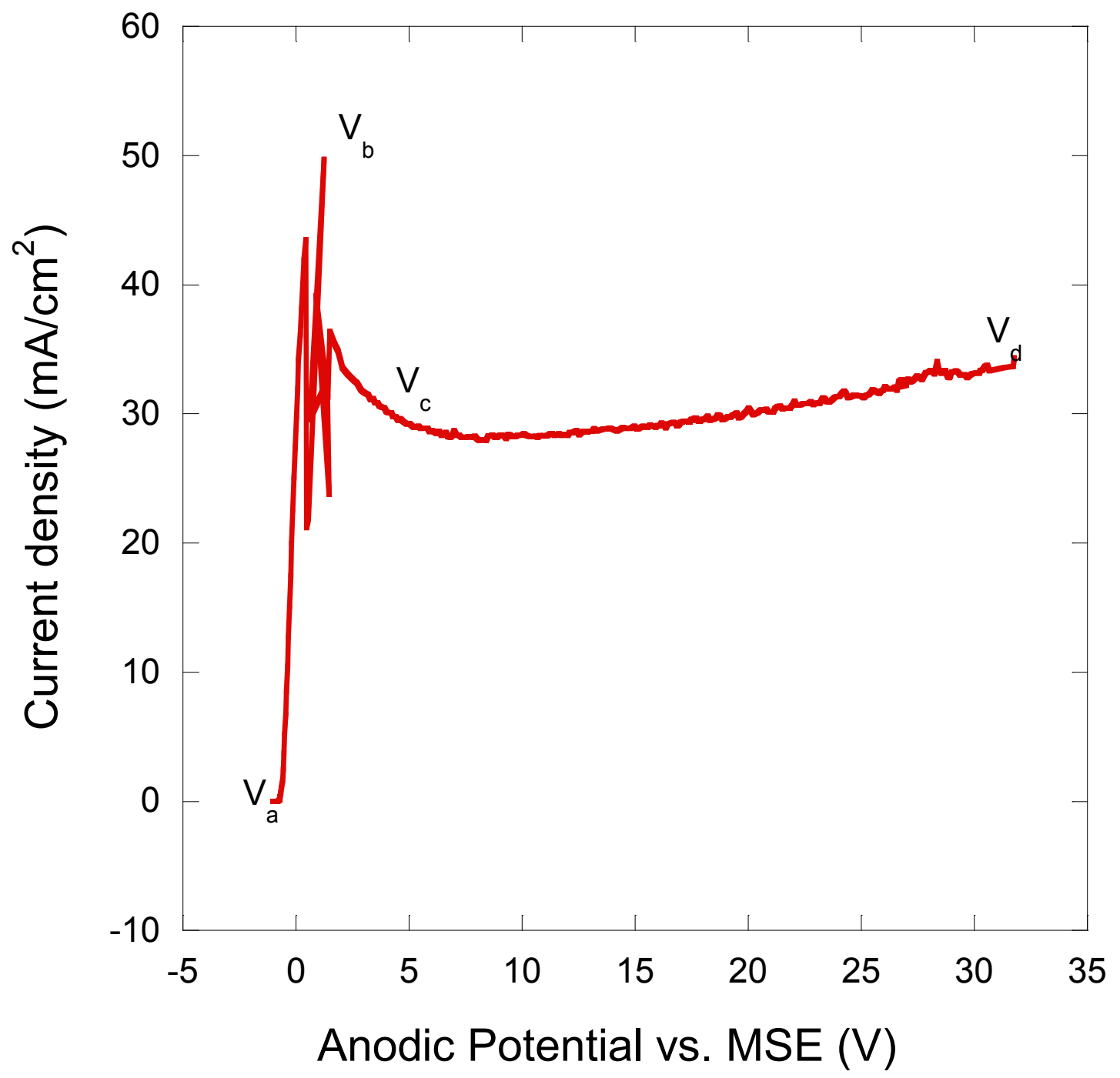

Fig. 4.1: General Nb electrochemical polarization curve. 


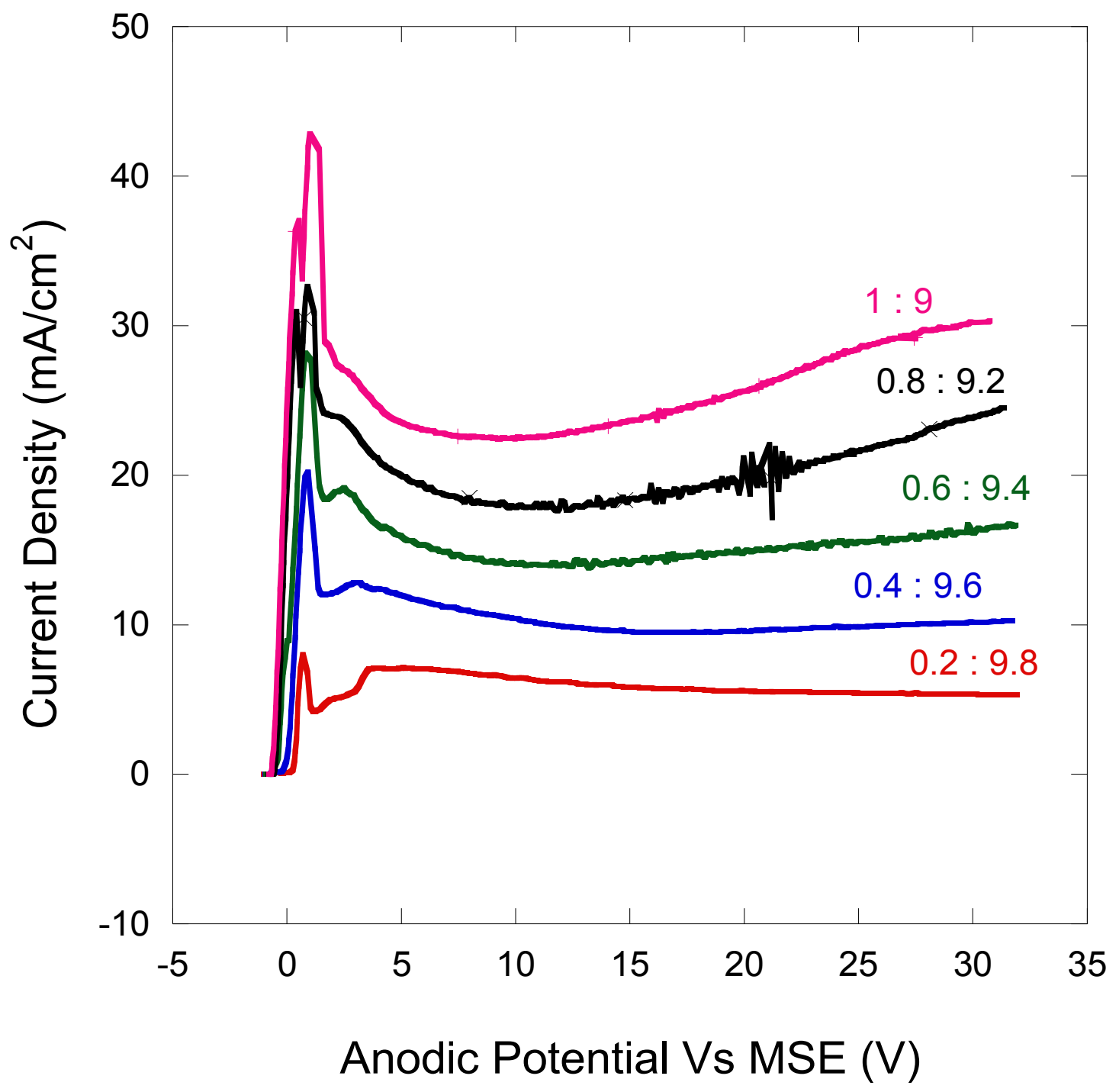

Fig. 4,2: Effect of acid ratio on $\mathrm{Nb}$ polarization curve at a temperature of $26^{\circ} \mathrm{C}$ 


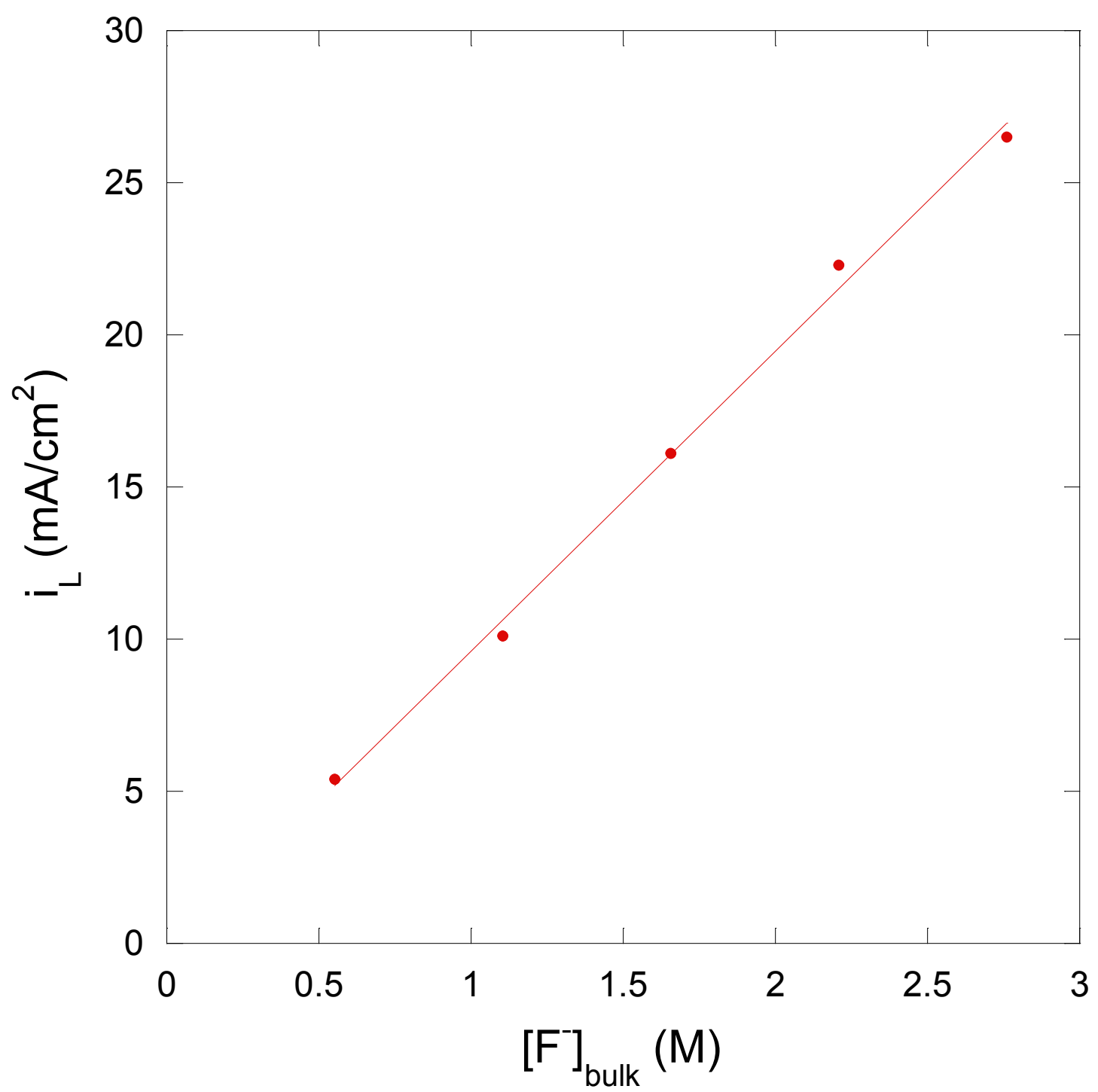

Fig. 4.3: Linear variation of limiting current density with bulk fluoride ion concentration. 


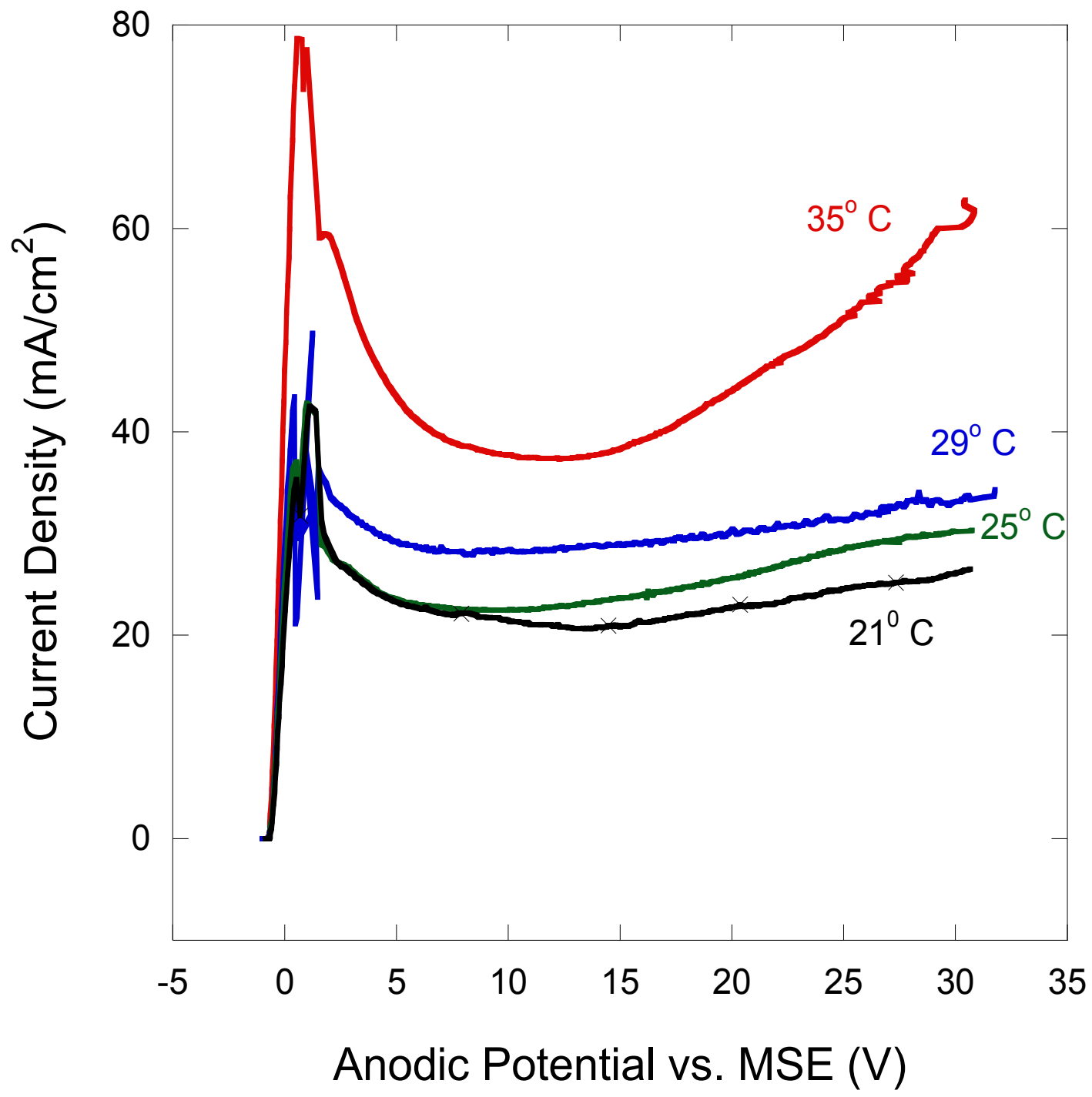

Fig. 4.4: Effect of temperature on the Nb polarization curve for standard electrolytic ratio 


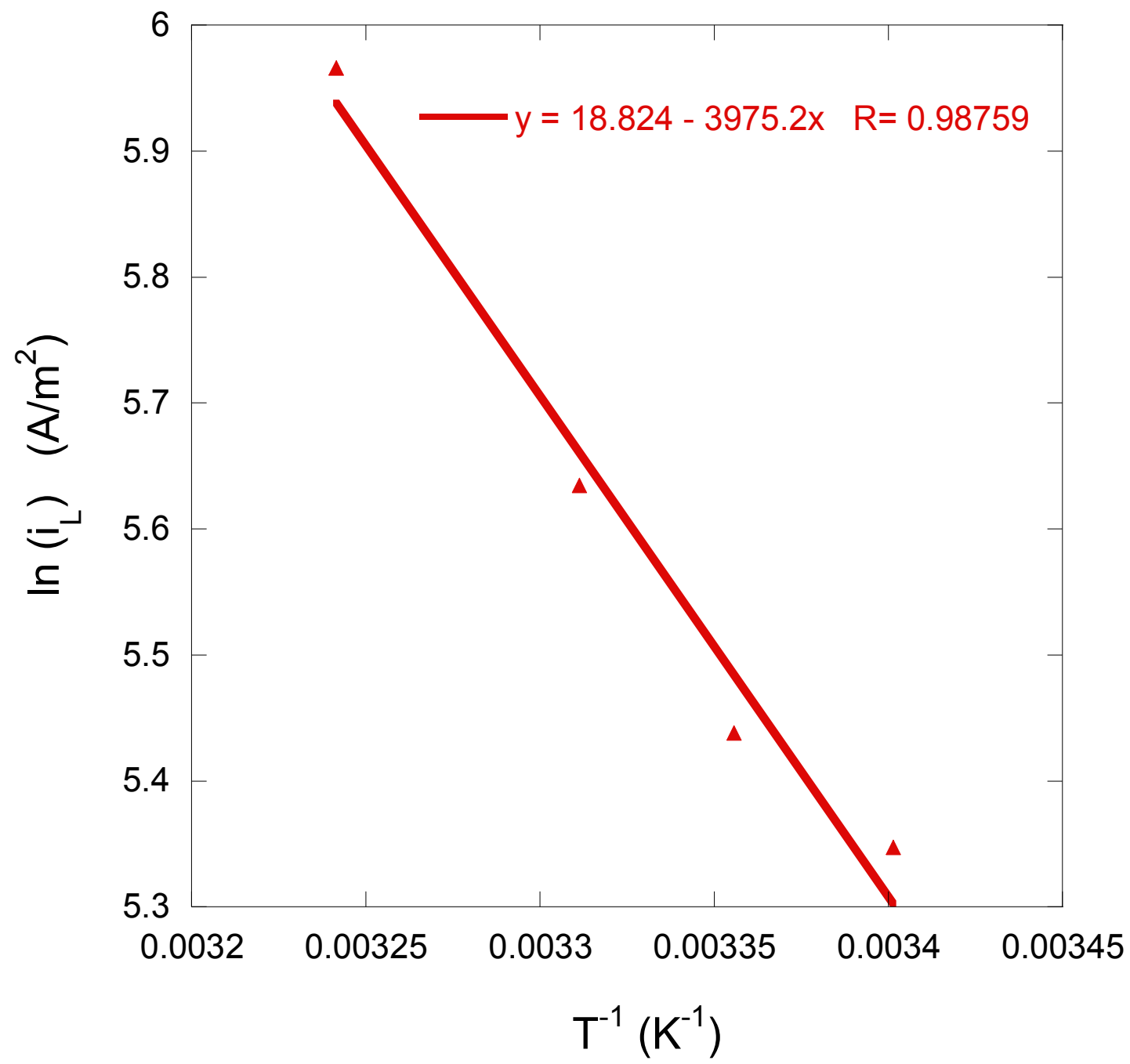

Fig. 4.5: Limiting current density variation with temperature. 


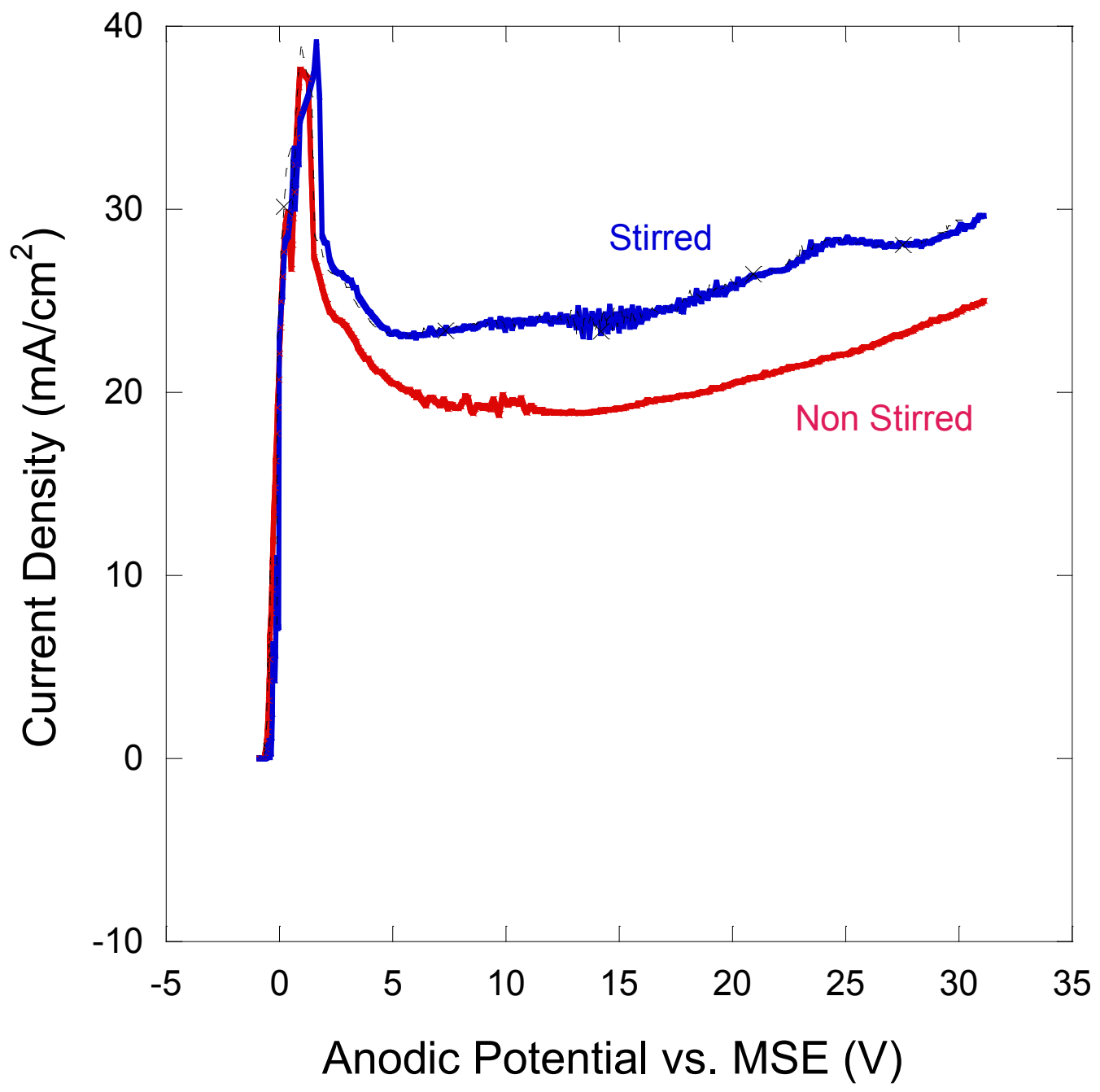

Fig. 4.6: Effect of stirring on $\mathrm{Nb}$ polarization curve. 


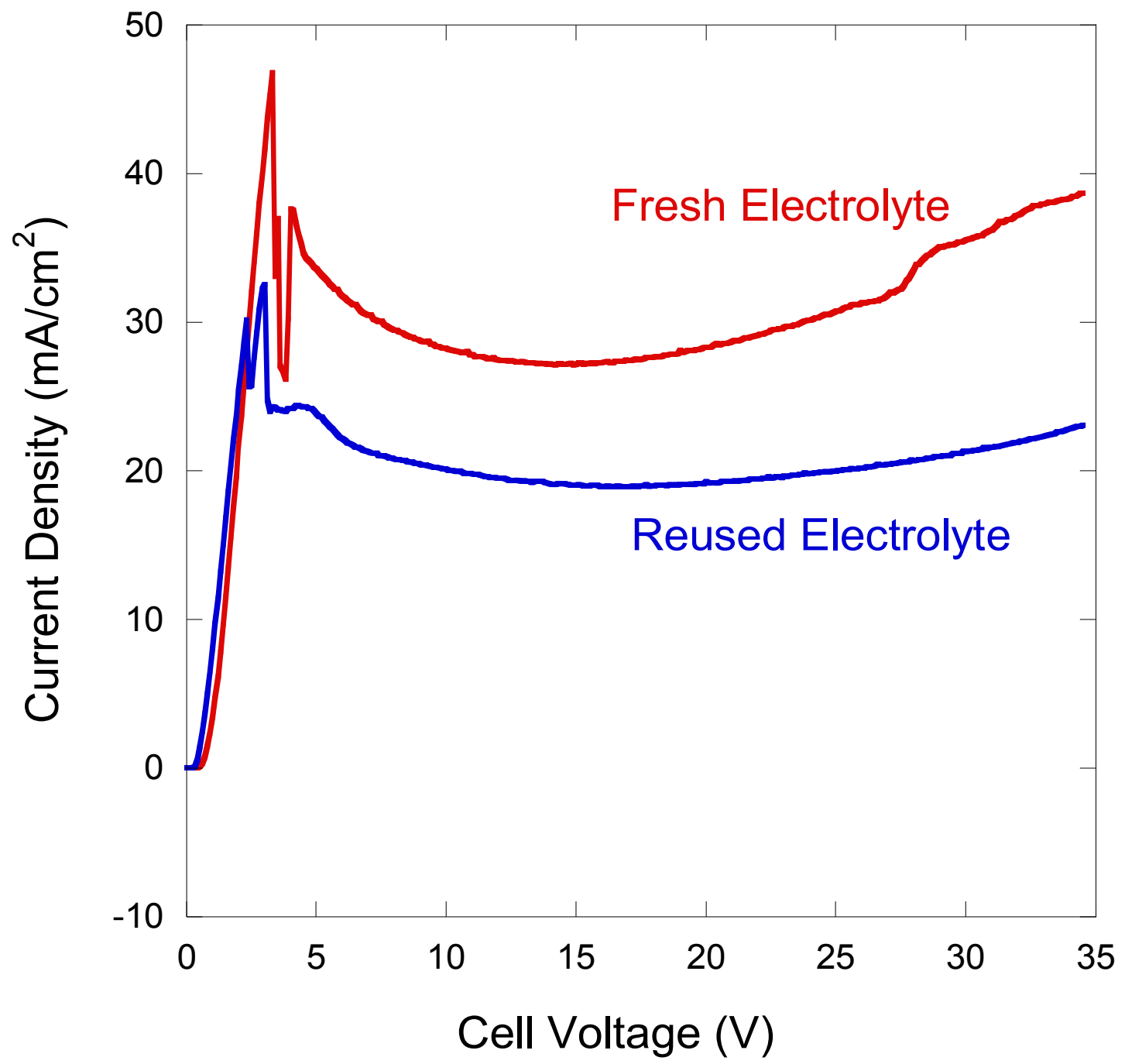

Fig. 4.7: Effect of reusing electrolyte on $\mathrm{Nb}$ polarization curve. 


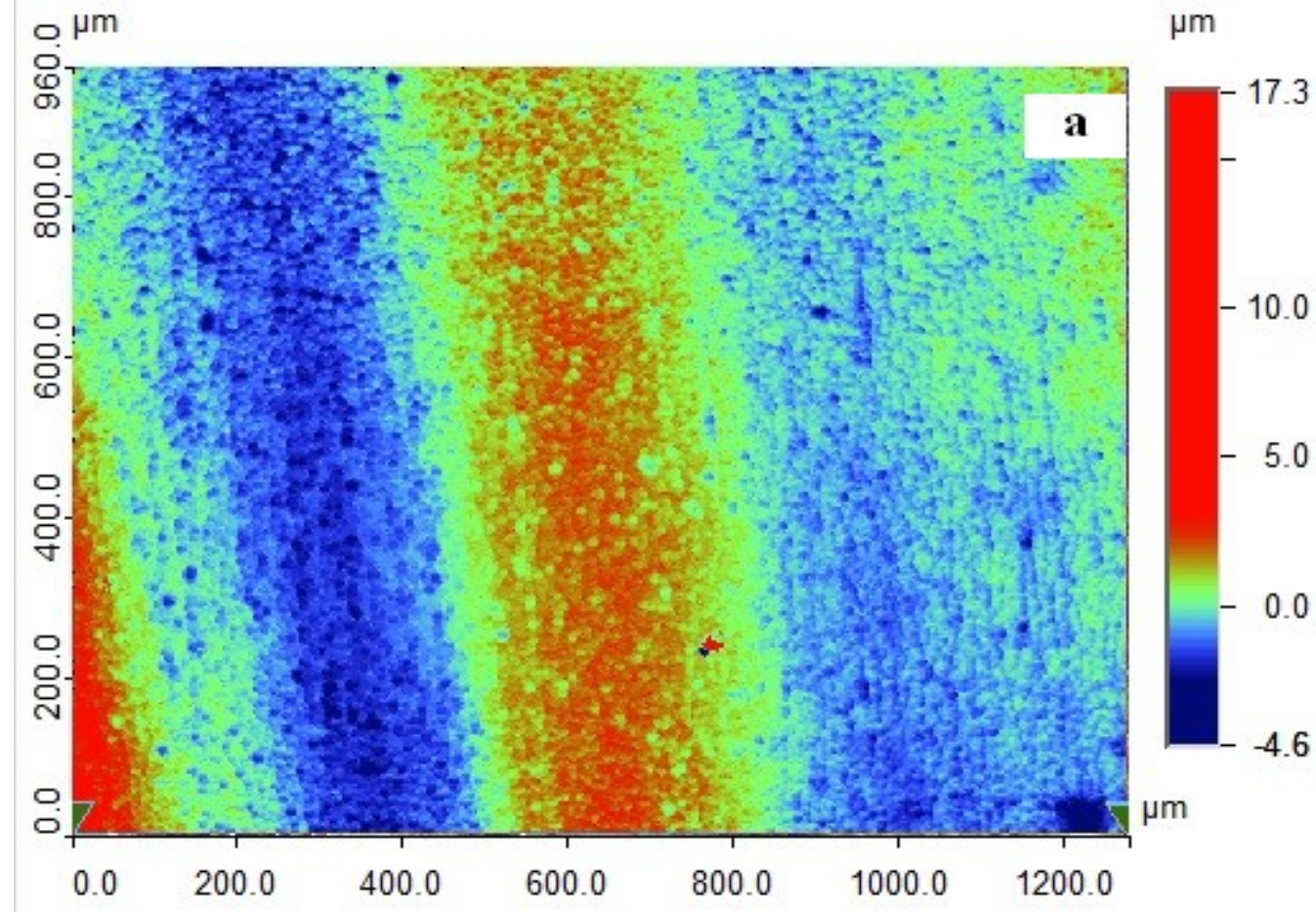

a)

Fig. 4.8a) Surface topography of $\mathrm{Nb}$ electropolished at an applied cell voltage of $5 \mathrm{~V}$ for $1 \mathrm{~h}$ at $26^{\circ} \mathrm{C}$ in standard electrolyte.

b) Surface topography of $\mathrm{Nb}$ electropolished at an applied cell voltage of $10 \mathrm{~V}$ for $1 \mathrm{~h}$ at $26^{\circ} \mathrm{C}$ in standard electrolyte.

c) Surface topography of $\mathrm{Nb}$ electropolished at an applied cell voltage of $15 \mathrm{~V}$ for $1 \mathrm{~h}$ at $26^{\circ} \mathrm{C}$ in standard electrolyte.

d) Surface topography of $\mathrm{Nb}$ electropolished at an applied cell voltage of $20 \mathrm{~V}$ for $1 \mathrm{~h}$ at $26^{\circ} \mathrm{C}$ in standard electrolyte.

Continued 
Fig 4.8. Continued

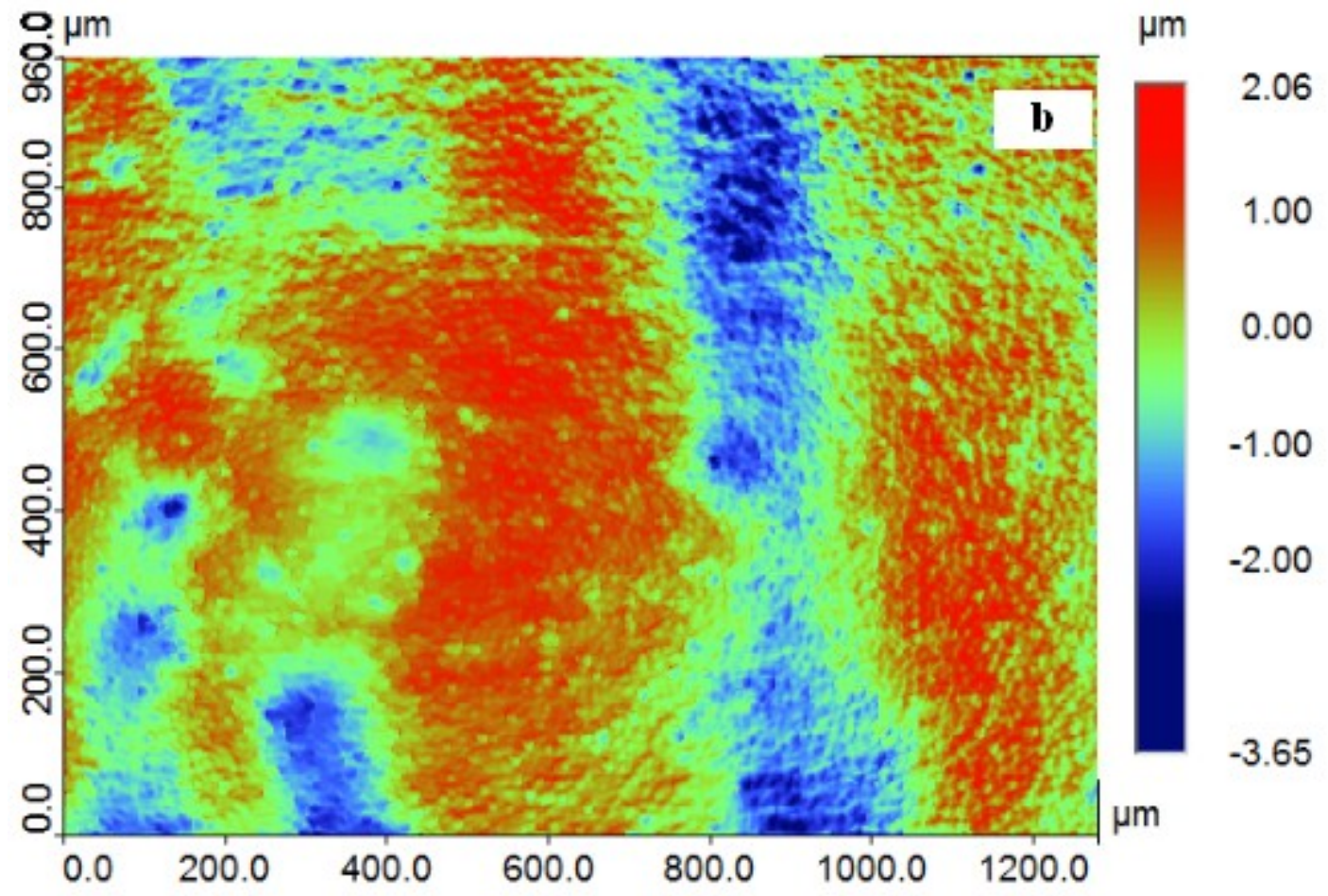

b)

Continued 
Fig 4.8. Continued

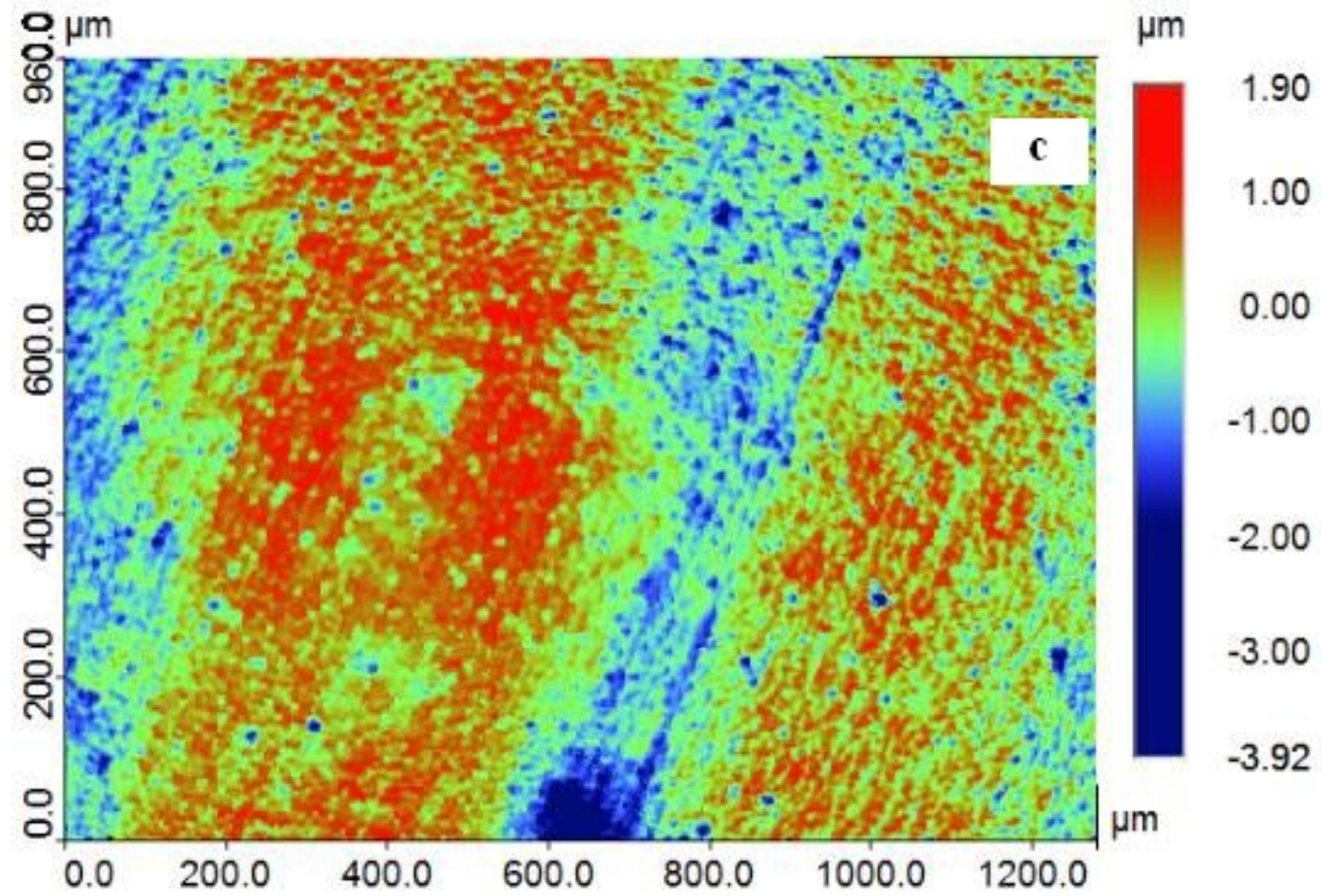

c)

Continued 
Fig 4.8. Continued

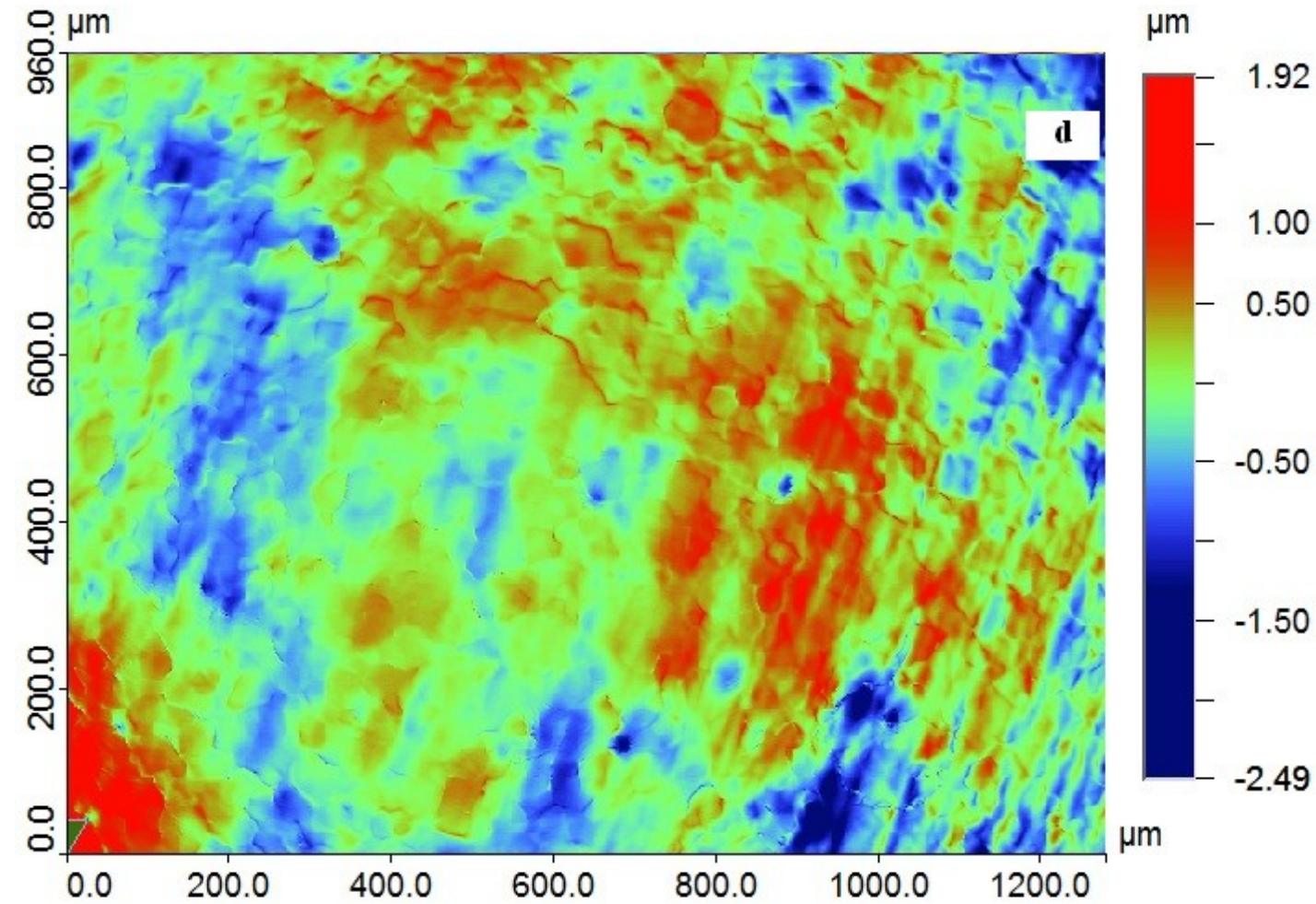

d) 


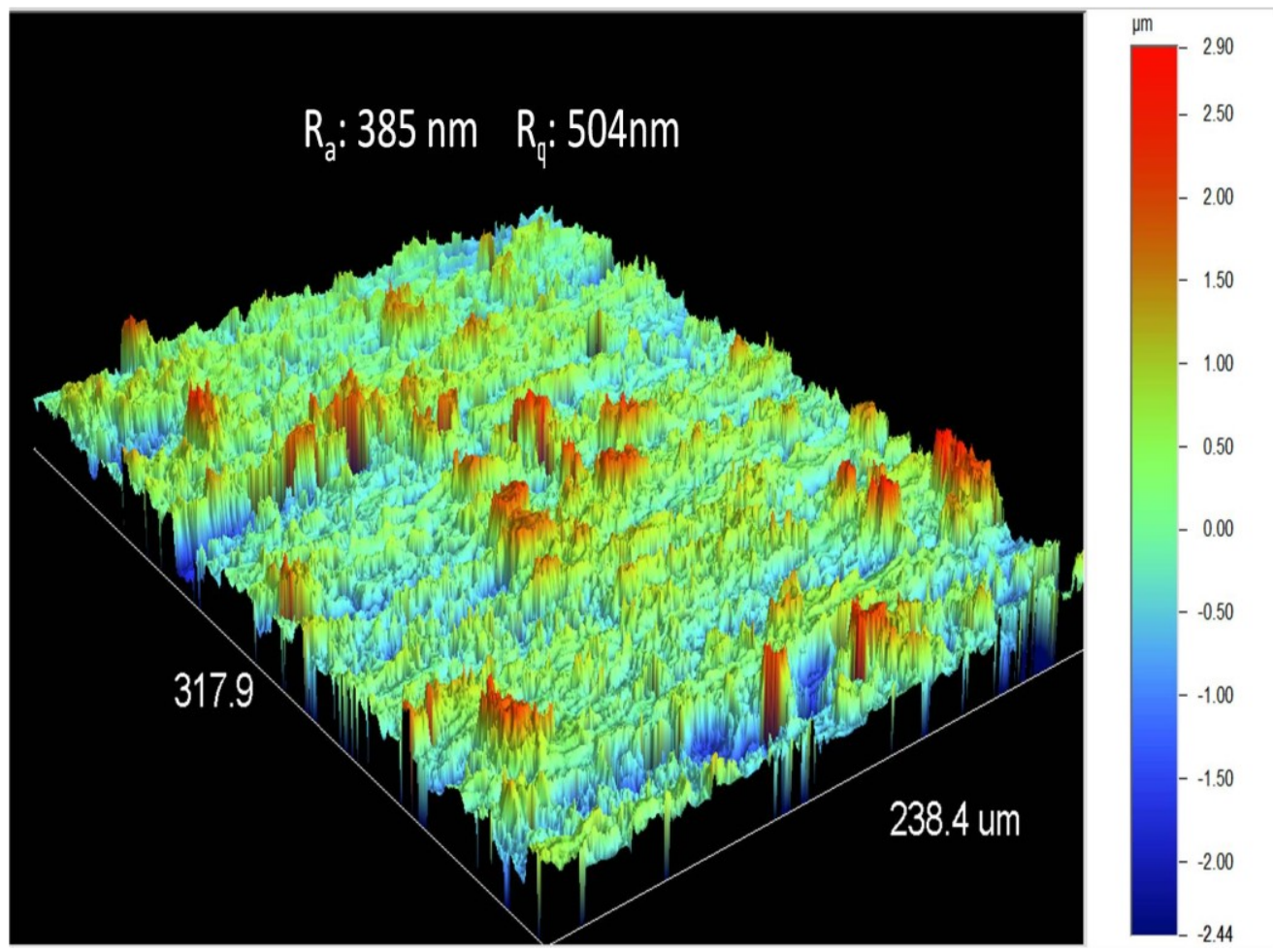

a)

Fig. 4.9a) Surface topography of niobium before electropolishing.

b) 3D Surface topography of $\mathrm{Nb}$ electropolished at an applied cell voltage of $15 \mathrm{~V}$ for $2 \mathrm{~h}$ at $26^{\circ} \mathrm{C}$ in standard electrolyte.

c) 3D Surface topography of $\mathrm{Nb}$ electropolished at an applied cell voltage of $15 \mathrm{~V}$ for $4 \mathrm{~h}$ at $26^{\circ} \mathrm{C}$ in standard electrolyte.

d) 3D Surface topography of $\mathrm{Nb}$ electropolished at an applied cell voltage of $15 \mathrm{~V}$ for $6 \mathrm{~h}$ at $26^{\circ} \mathrm{C}$ in standard electrolyte. 
Fig 4.9. Continued
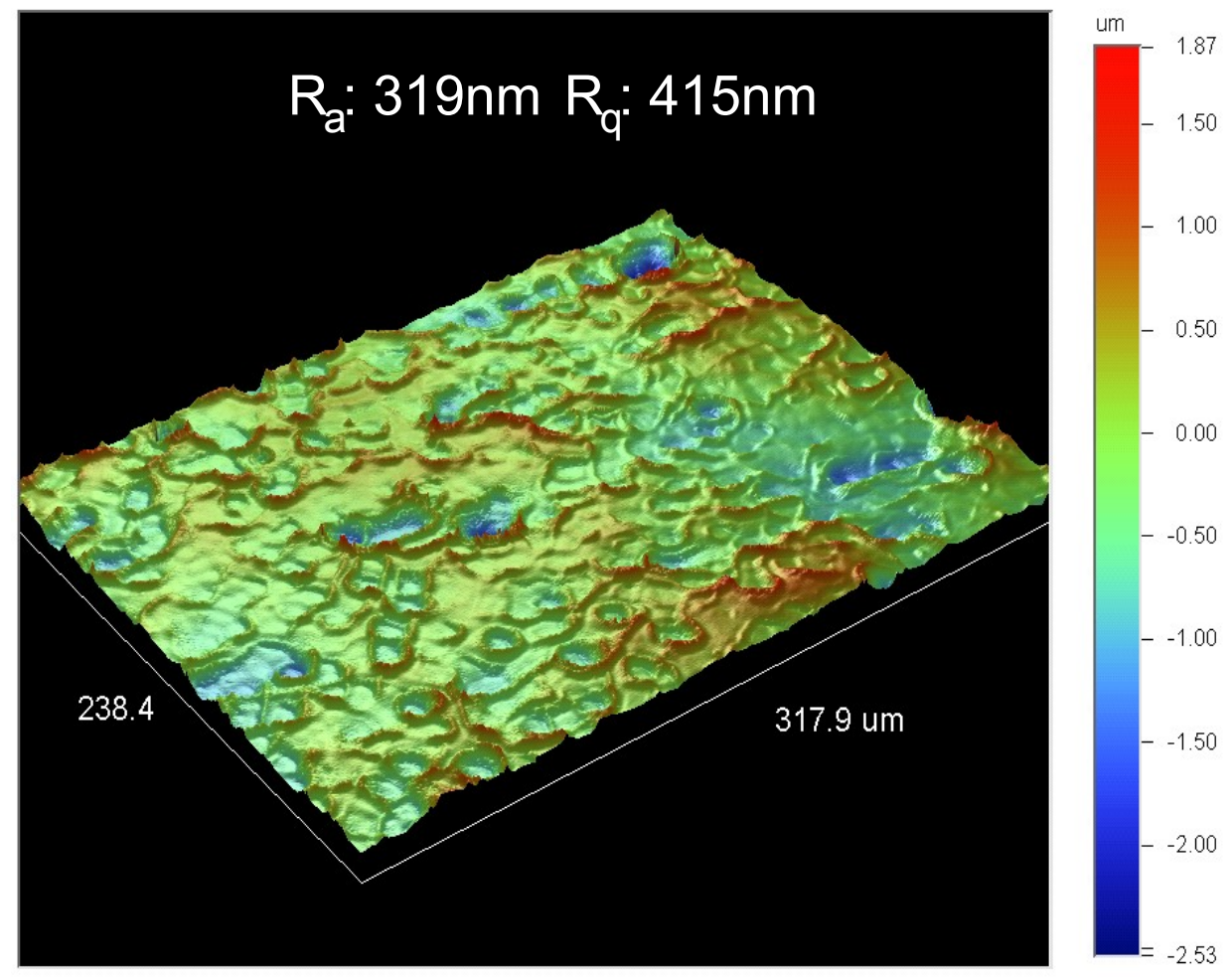

b)

Continued 
Fig 4.9. Continued

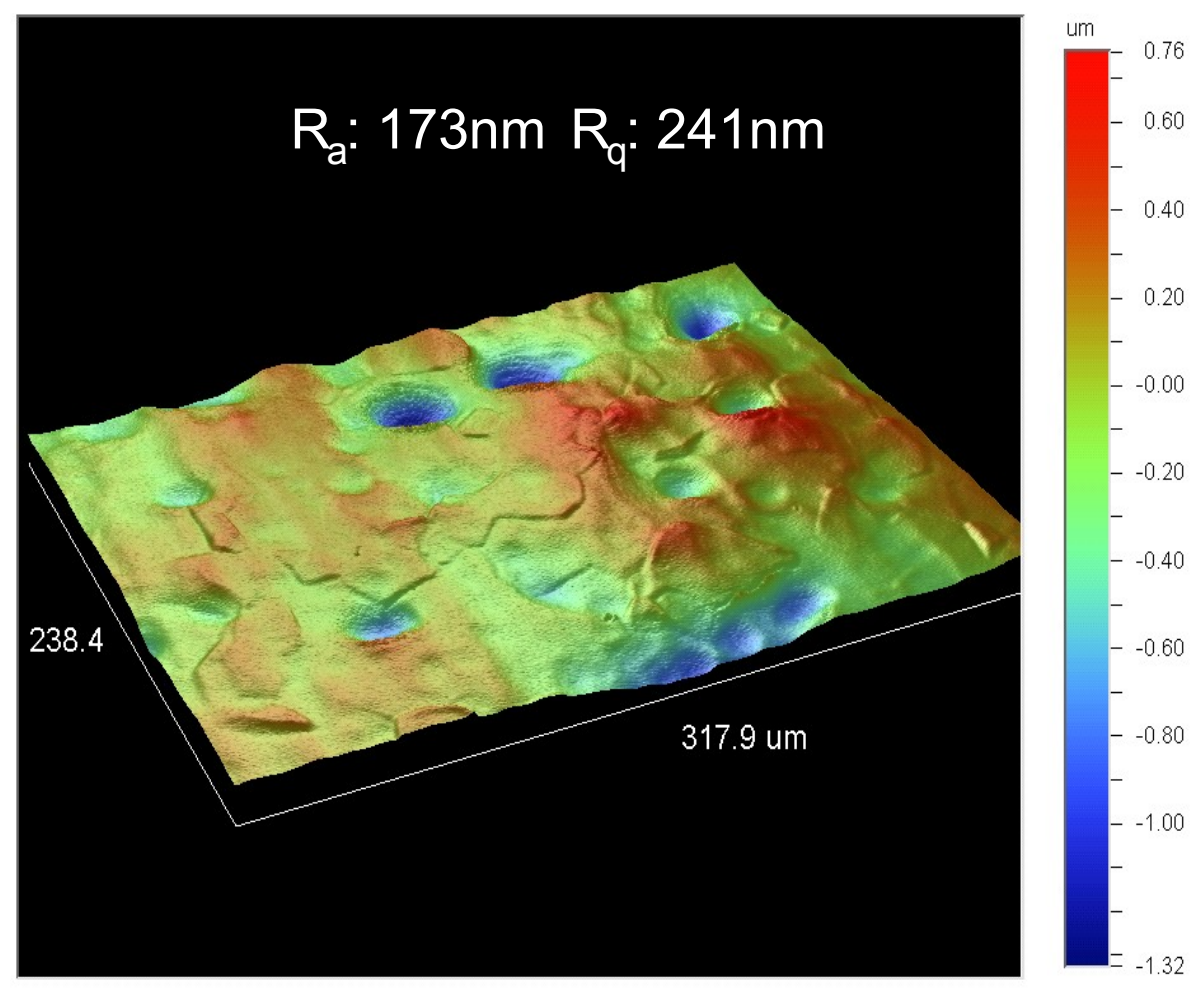

c)

Continued 
Fig 4.9. Continued

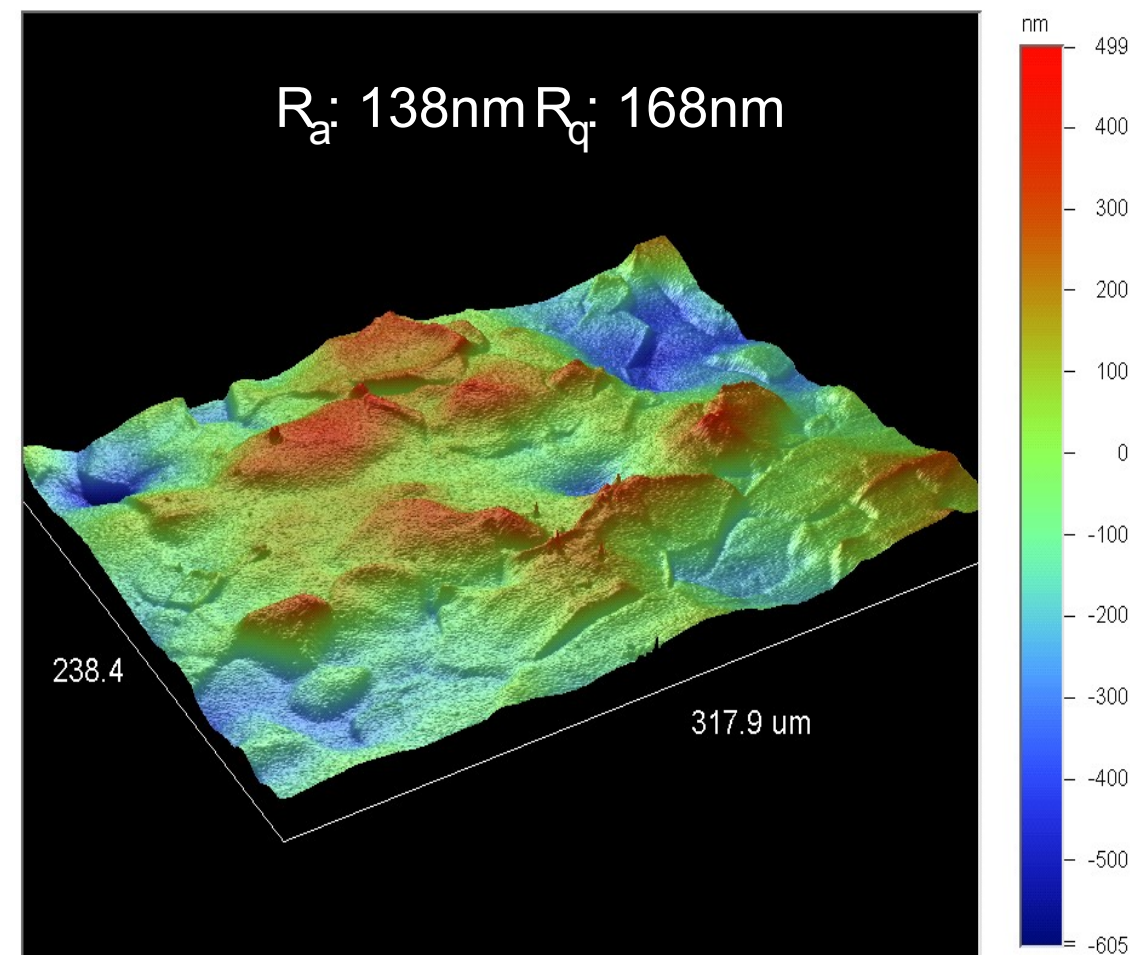

d) 


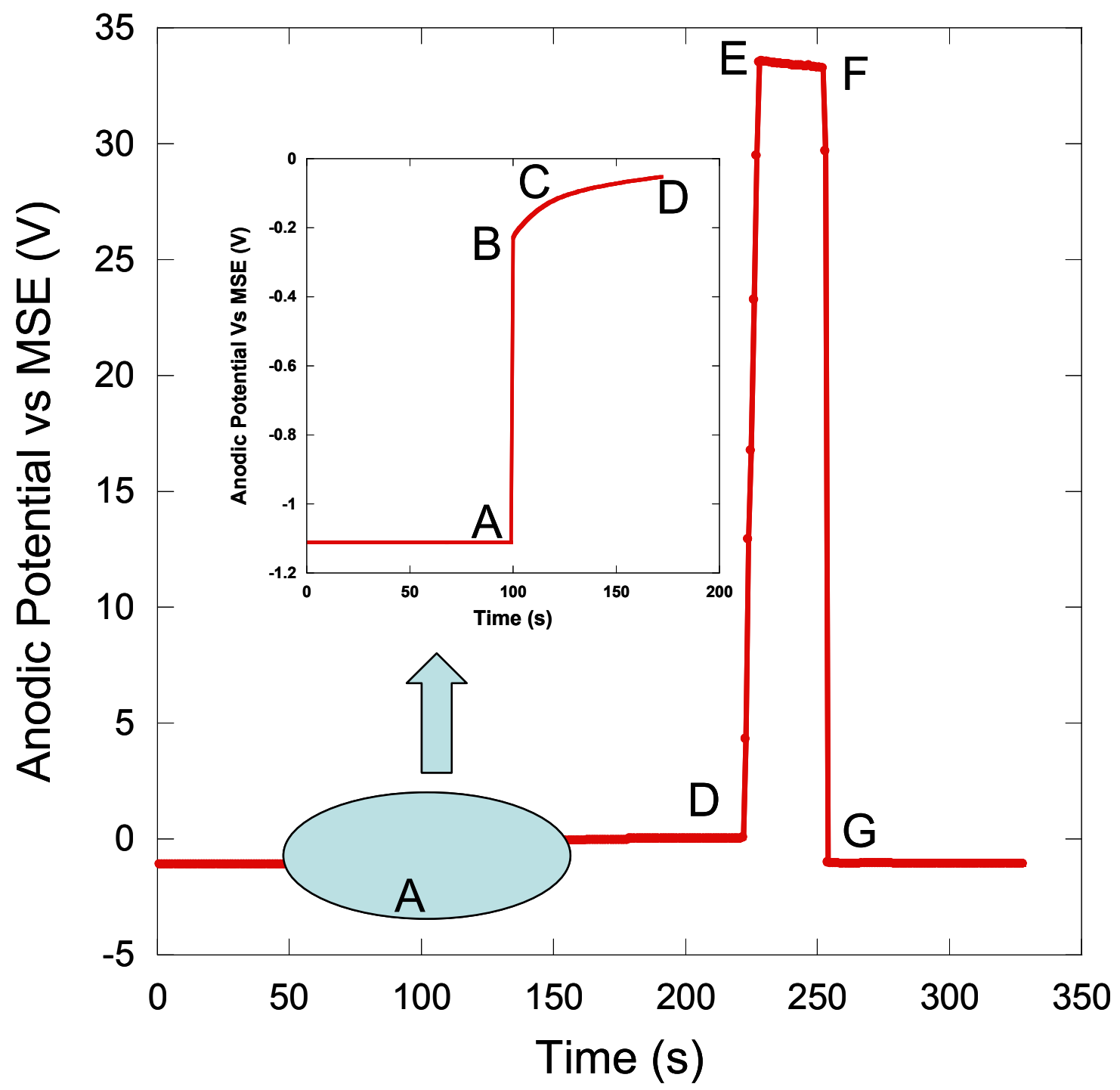

Fig. 4.10: Potential transient for an applied current density on or above the limiting current density. 


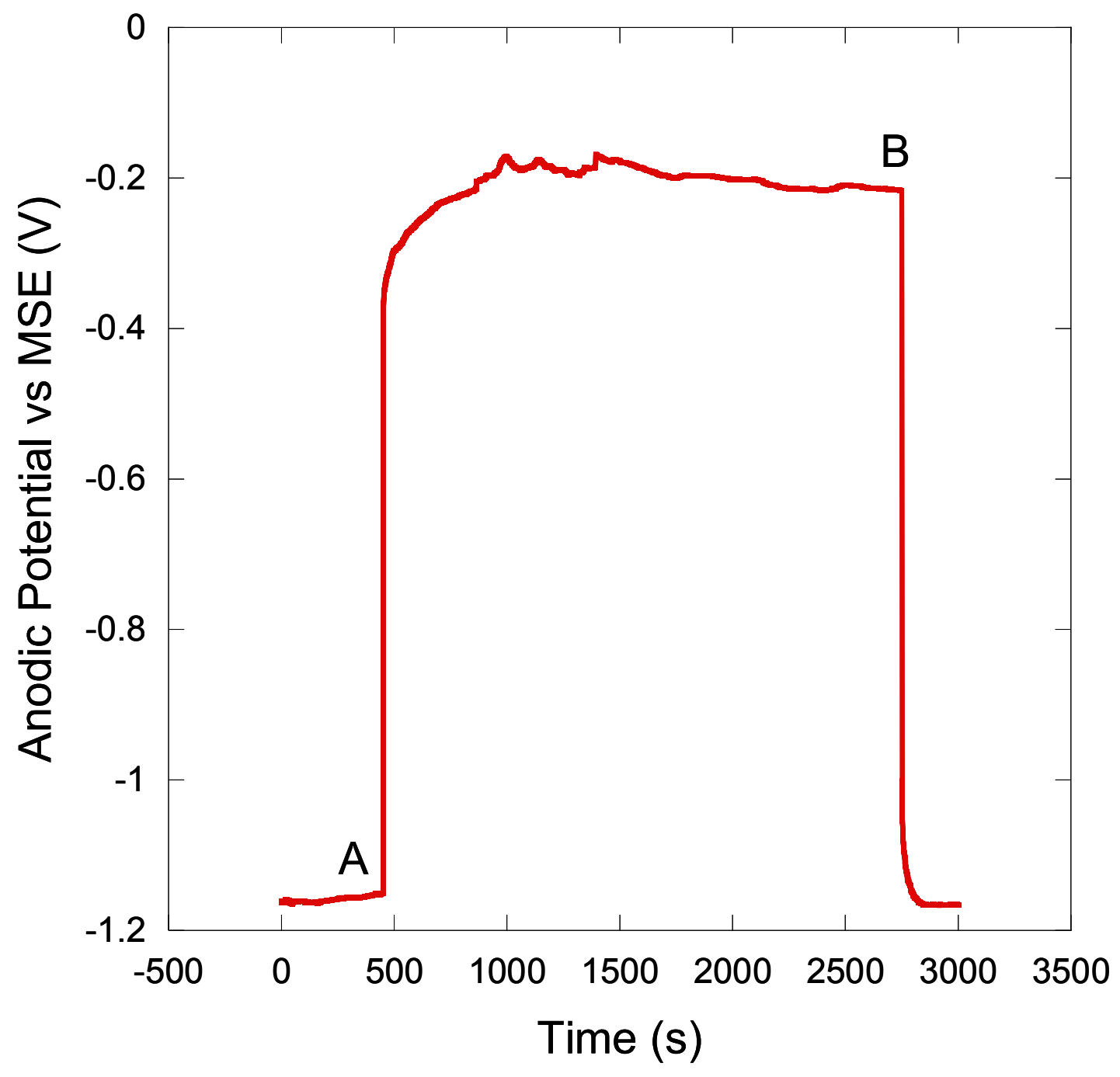

Fig. 4.11: Potential transient for an applied current density below the limiting current density. 


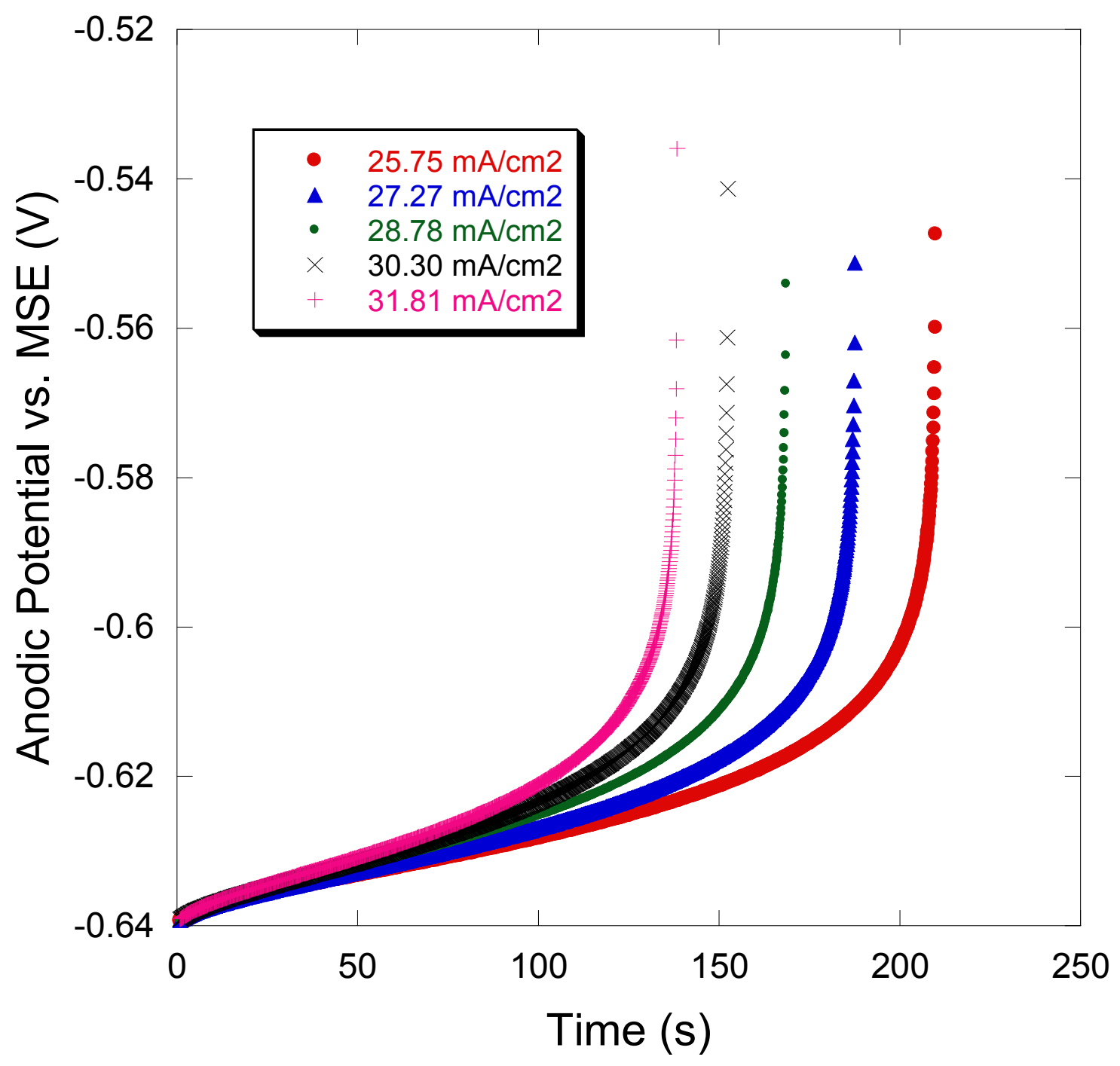

Fig. 4.12: Simulated variation of concentration polarization with time 


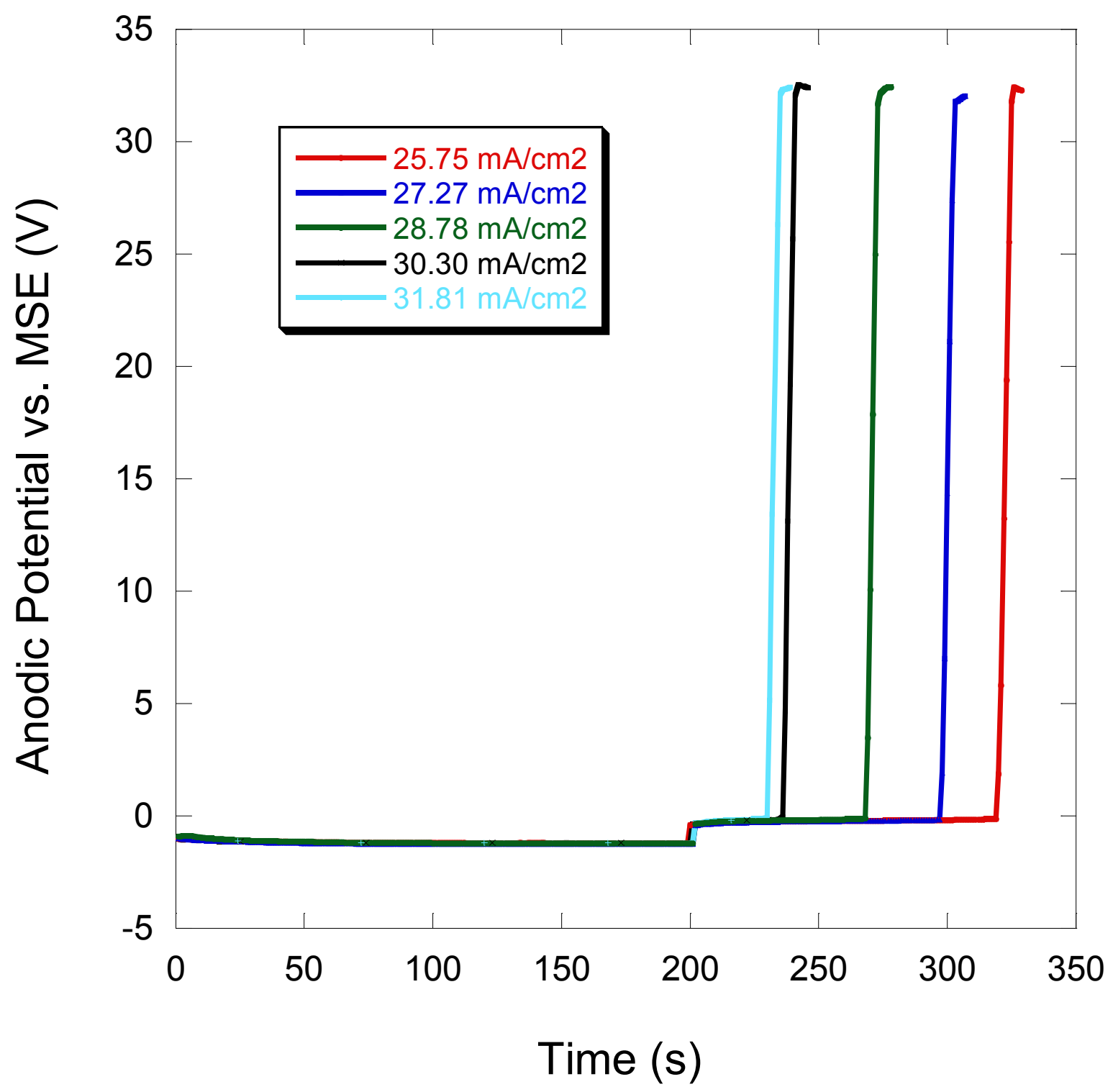

Fig. 4.13: Potential transients demonstrating the increase in transient time with decreasing current density. 


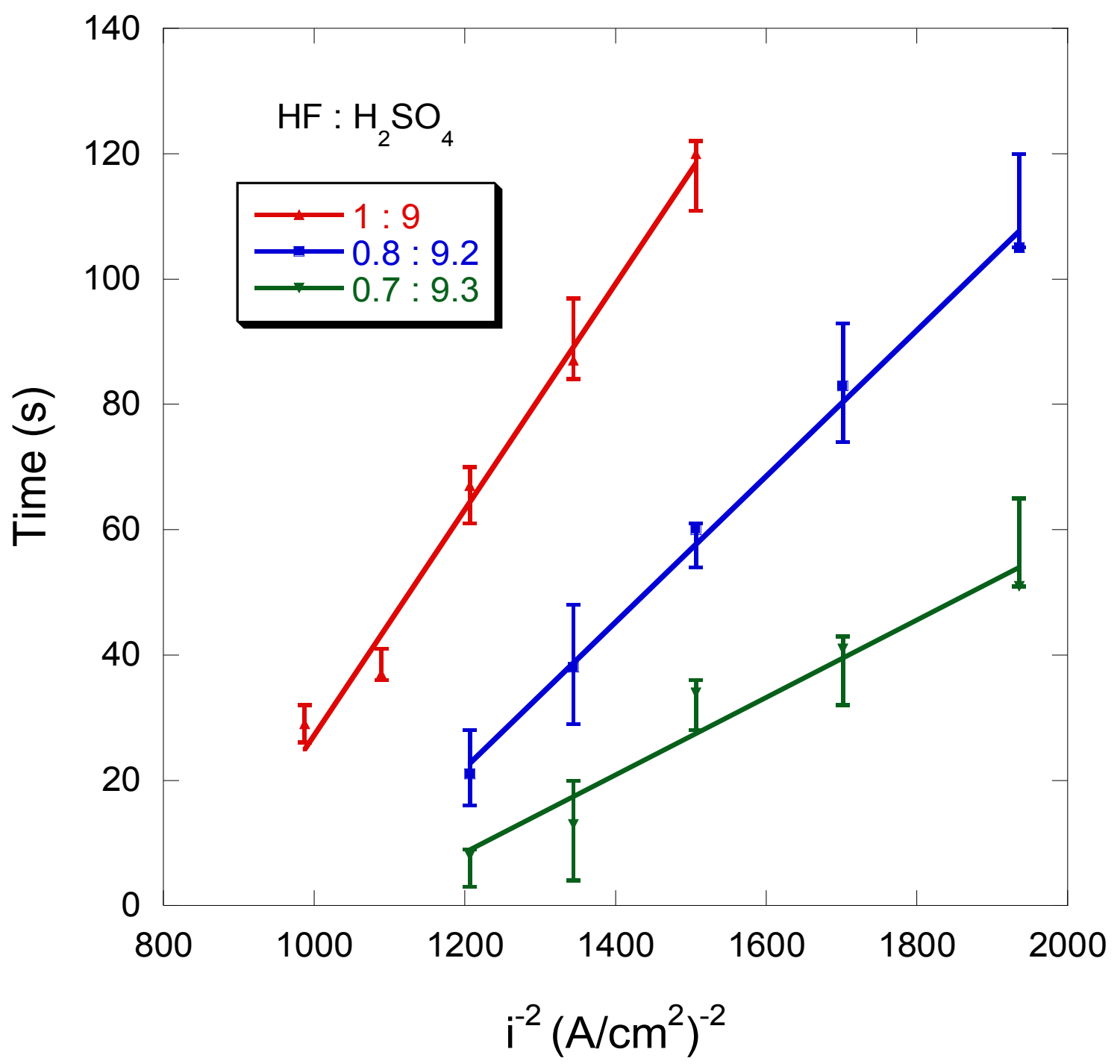

Fig. 4.14: Effect of current density on transition time for constant acid ratio. 


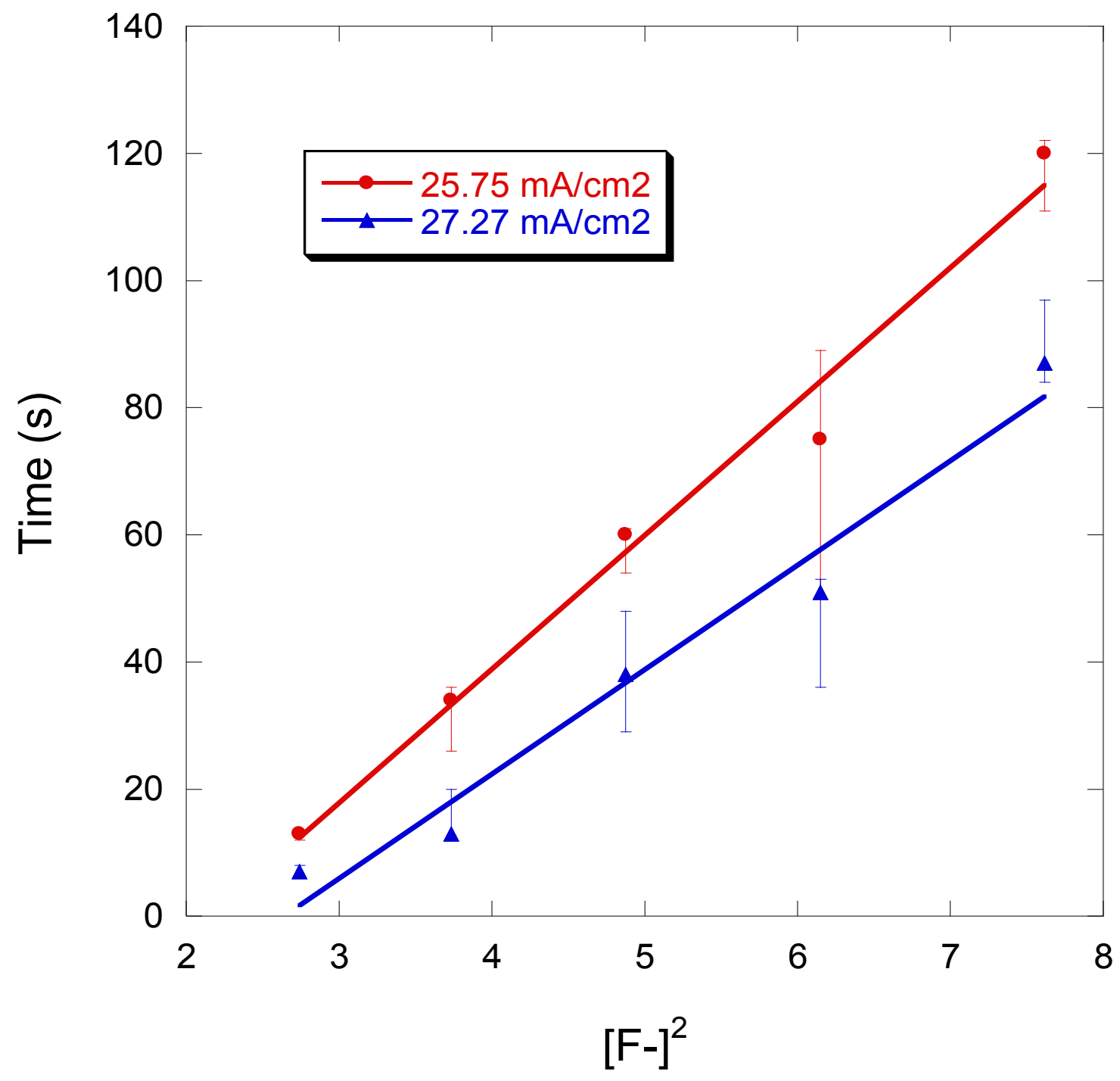

Fig. 4.15: Effect of fluoride ion concentration on transition time for a constant applied current density 

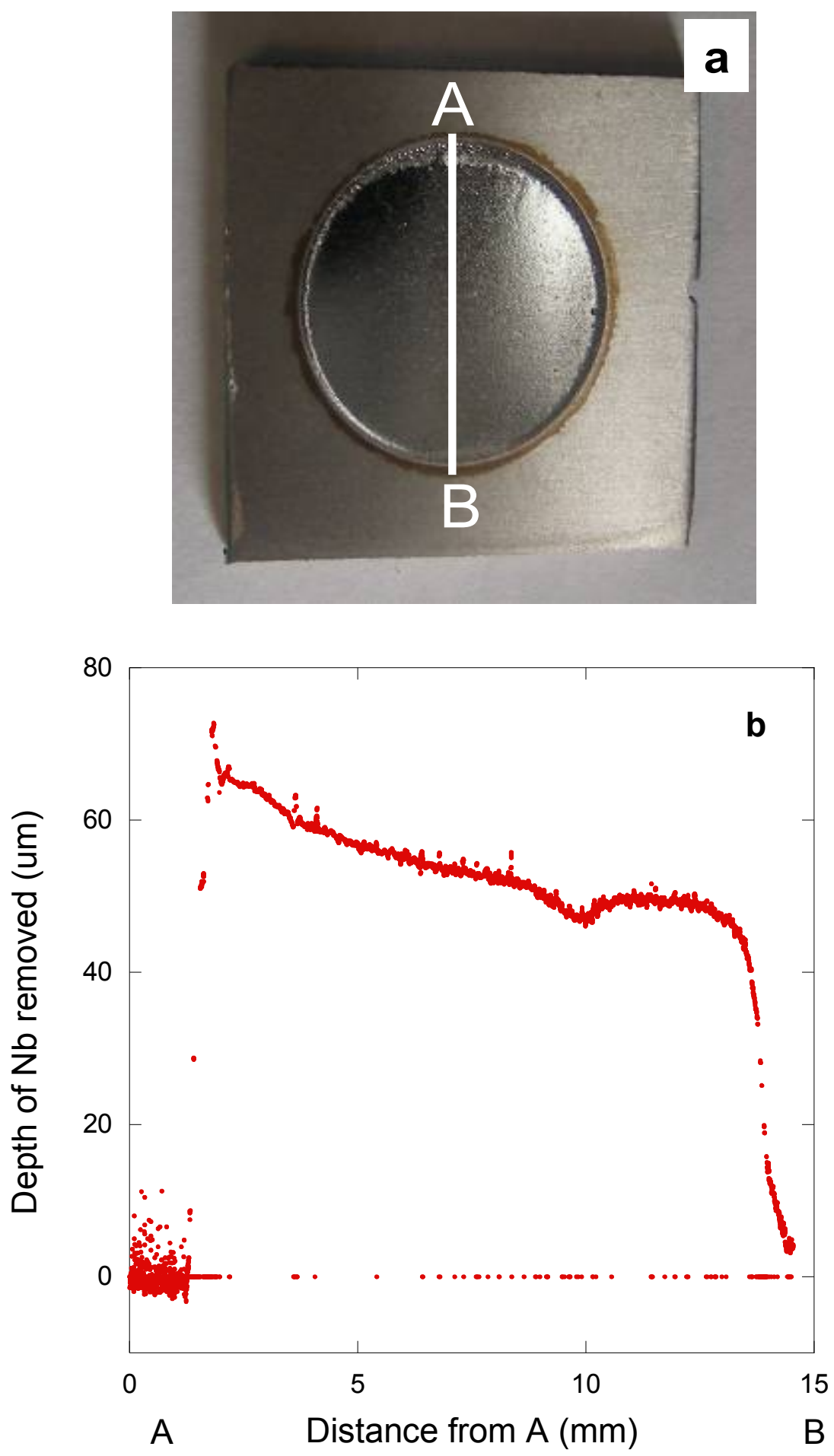

Fig. 4.16: (a) Masked Nb sample electropolished in the exposed circular area.

(b) Variation of the depth of niobium removed along $\mathrm{AB}$. 


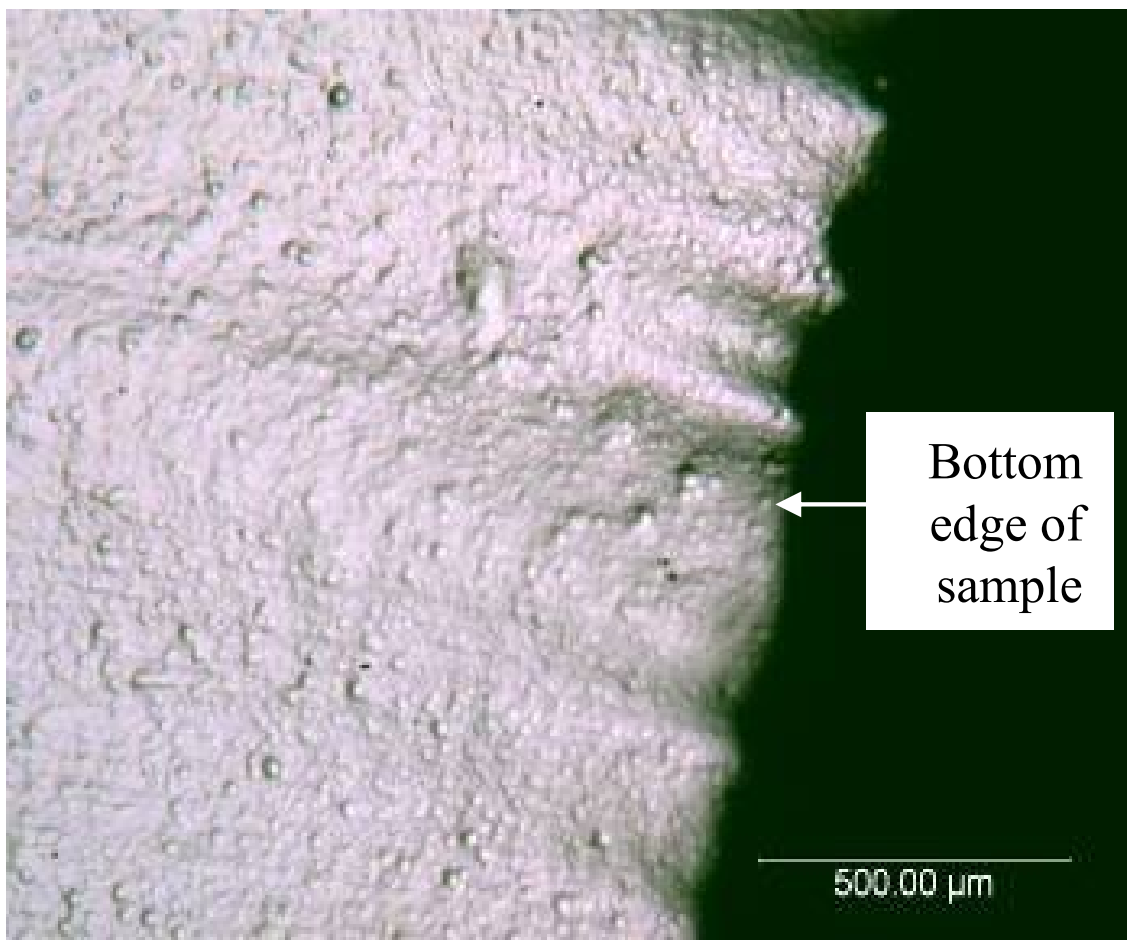

Fig. 4.17: Ridges observed at the bottom edge of flag electrode sample. 


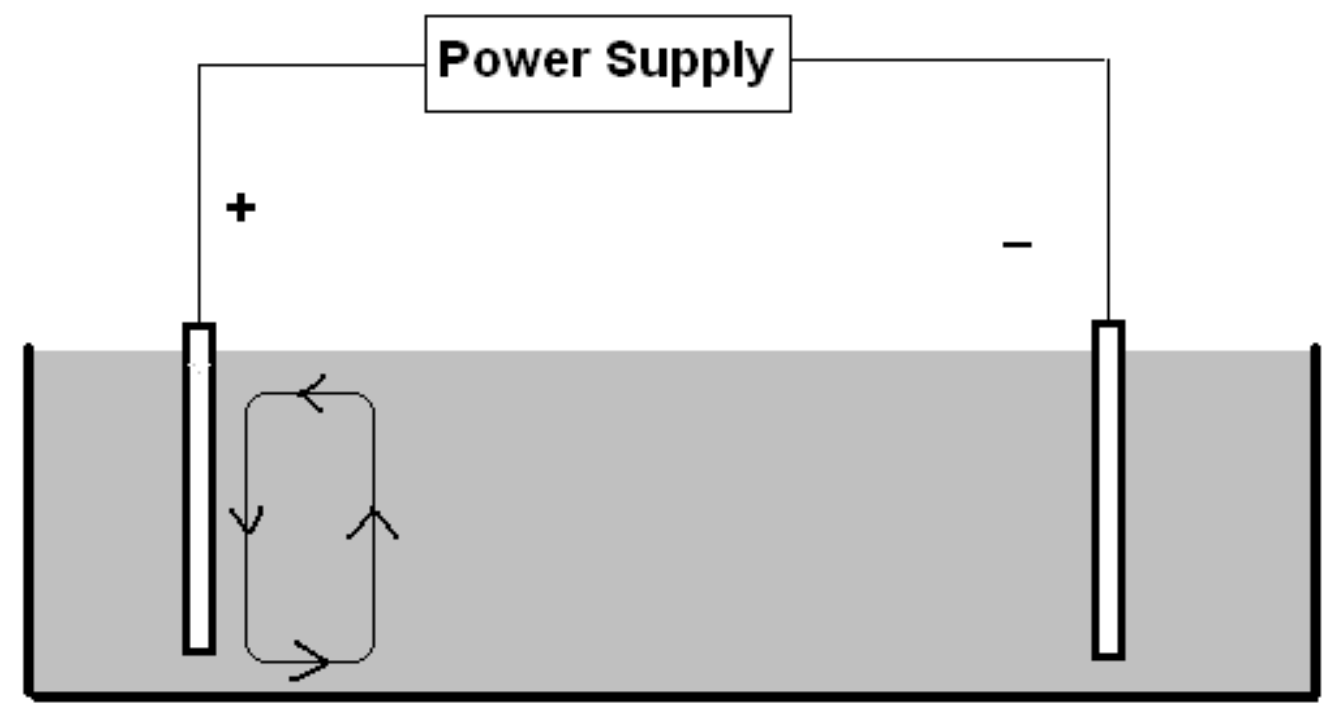

Fig. 4.18: Schematic depicting natural convection resulting from denser electrolyte near the working electrode surface because of salt film formation. 


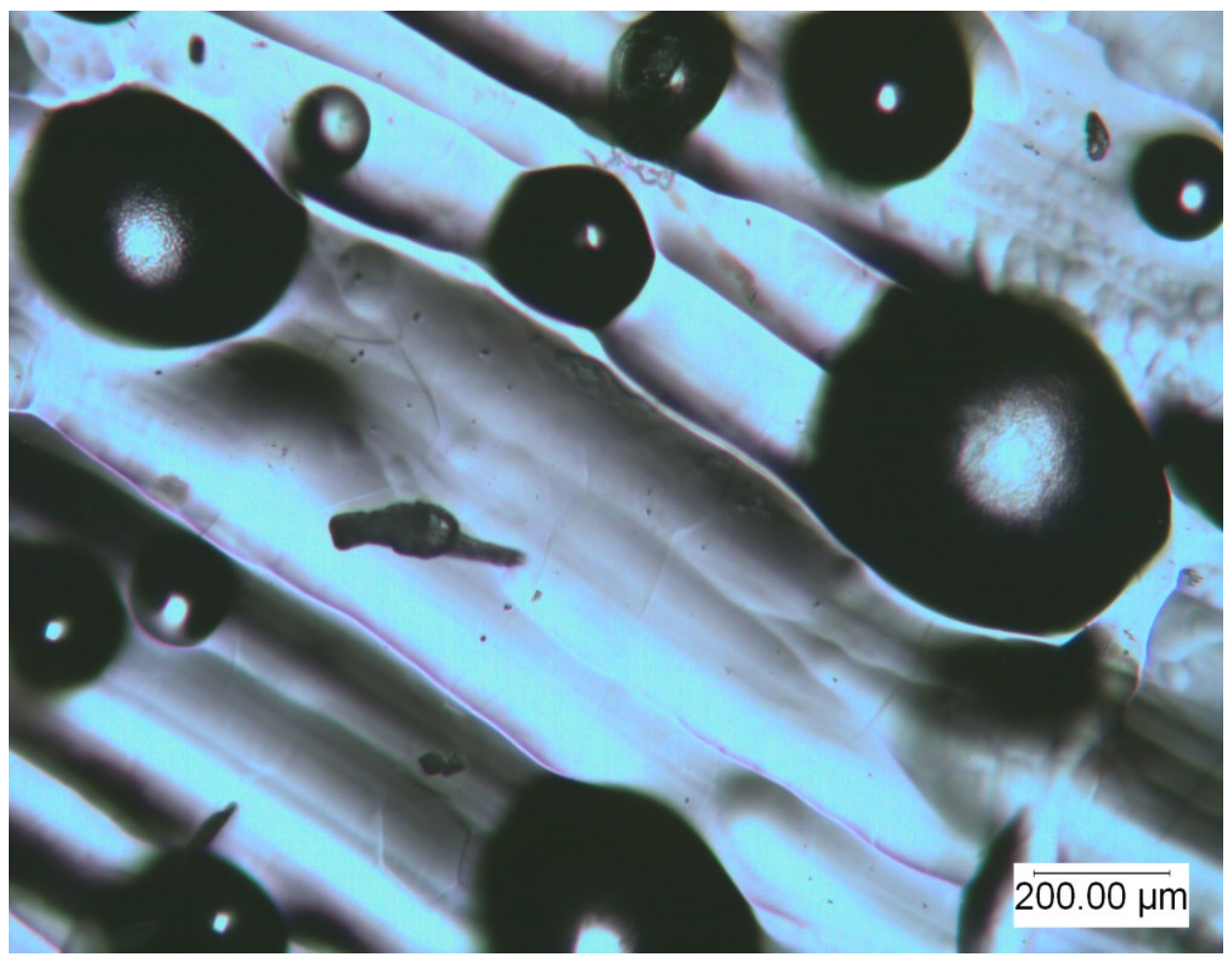

Fig. 4.19: Pits at the bottom surface of an electropolished $\mathrm{Nb}$ flag electrode sample. 


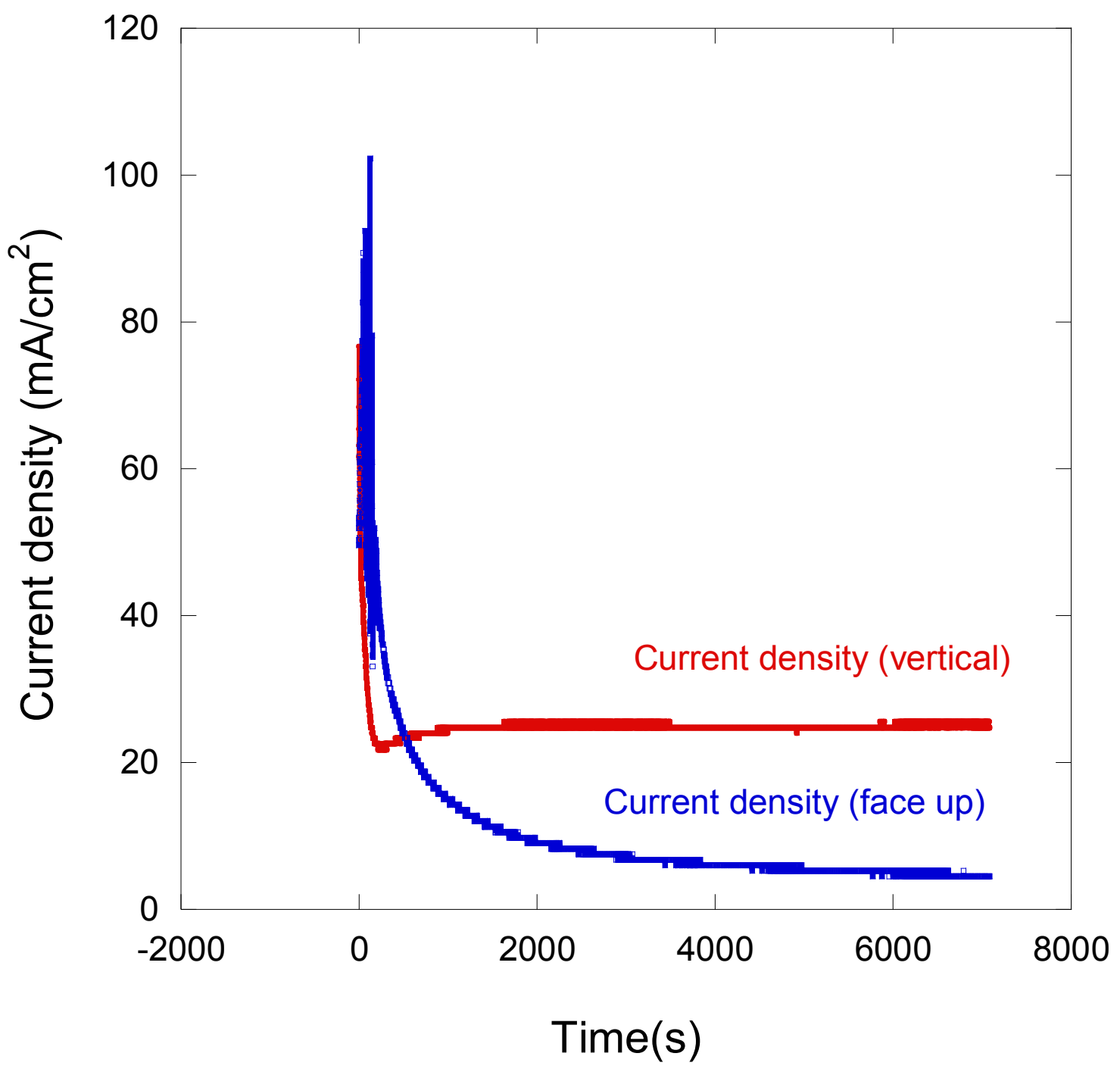

Fig. 4.20: Current transients emphasizing the effect of sample configuration in the electrolyte. 


\subsection{References}

1. Frankel, G., MSE 735 Kinetics II. 2010, Fontana Corrosion Center: Columbus

2. Palmieri, V. Fundamentals of Electrochemistry - The Electrolytic polishing of Metals: Application to Copper and Niobium. in SRF Workshop 2003. 2003. Germany.

3. H.Padamsee, RF Superconductivity : Science, Technology, and Applications. 2009, Weinheim: Wiley-VCH.

4. Tian, H., et al., The mechanism of electropolishing of niobium in hydrofluoricsulfuric acid electrolyte. Journal of the Electrochemical Society, 2008. 155(9): p. D563-D568.

5. Bard, A.J. and L.R. Faulkner, Electrochemical Methods: Fundamentals and Applications. 1980, New York: John Wiley \& Sons.

6. William D. Callister, J., Materials Science And Engineering An Introduction. 7 ed. 2007, New York: John Wiley \& Sons.

7. Tian, H., M.J. Kelley, and C.E. Reece, Evaluation of the fluoride ion diffusion coefficient in a sulfuric and hydrofluoric acid electrolyte during niobium electropolishing for superconducting radio frequency (SRF) accelerator cavities. Abstracts of Papers of the American Chemical Society, 2009. 238.

8. Eozenou, F., et al., Electropolishing of Niobium: best EP Parameters, in CAREReport-06-010-SRF. 2006. 
9. H.Padamsee, J.Knobloch, and T.Hays, $R F$ Super-conductivity for Accelerators. 1998, New York: John Wiley \& Sons.

10. Bockris, J.M. and A.K.N. Reddy, Modern Electrochemistry-Ionics. Vol. 1. 2002, New York: Kluwer Academic Publishers. 1232-1234.

11. Bard, A.J. and L.R. Faulkner, Electrochemical Methods Fundamentals and Applications. 2 ed. 2001, Hoboken, NJ: John Wiley \& Sons.

12. Matlosz, M., Modeling of Impedance Mechanisms in Electropolishing. Electrochimica Acta, 1995. 40(4): p. 393-401.

13. Landolt, D., Corrosion and Surface Chemistry of Metals. 1 ed. 2007, Lausanne, Switzerland: EPFL Press.

14. H.C.Kuo and D. Landolt, Rotating-disk electrode study of anodic dissolution of iron in concentrated chloride media. Electrochimica Acta, 1975. 20(5): p. 393399. 


\section{Chapter 5 Conclusions}

The important parameters affecting the electropolishing of niobium and the mechanism of electropolishing were studied in this work. The effects were attributed to their influence on diffusion and convection, which are critical in the electropolishing process. Electrochemical polarization experiments helped determine the effects of temperature, electrolyte composition, convection and electrolyte age on the niobium electropolishing system. The limiting current density increased with increasing temperature of the electrolyte because of increasing diffusivity. Stirring of the electrolyte also resulted in an increase in the limiting current density because of a thinner diffusion layer. Limiting current density also increased with increasing $\mathrm{HF}: \mathrm{H}_{2} \mathrm{SO}_{4}$ ratio. This result indicated that fluoride ion was the transport limited species. Freshness of the electrolyte was found to be another important factor affecting the electropolishing rate. Freshly prepared electrolyte showed a higher limiting current density compared to the electrolyte that had been previously used. This could be related to the fluoride ion being used up in the course of electropolishing or loss of hydrofluoric acid because of evaporation owing to its low vapor pressure.

The applied cell voltage and the electropolishing duration were other important parameters affecting the process. The surface finish improved with higher applied cell voltage along the current density plateau while the other experimental parameters were

kept constant. The surface finish was also found to improve with greater duration of 
electropolishing. The rate of improvement in surface finish slows with time of electropolishing. This indicates a certain depth of material removal is required to attain a certain level of surface finish.

Galvanostatic experiments helped confirm that the mechanism of niobium electropolishing is diffusion limited transport of fluoride ions to the niobium surface. The validity of Sand's equation was tested to determine that fluoride ion is the rate limiting species. The transient time measurements showed a linear relation with inverse square of applied current density and with square of the fluoride ion concentration as predicted by Sand's equation. However neither curves passed through the origin, which is also predicted by Sand's equation. The slope of these curves indicated a higher diffusion coefficient than would be expected for a perfect semi-infinite one dimensional diffusion model. The higher diffusion coefficient could result from higher temperature in the vicinity of the electrode. Natural convection could be another factor contributing to the deviation from ideality.

Certain visual and experimental results indicate the presence of natural convection in the system. A bluish green layer was observed during niobium electropolishing, which vanished as soon as the current was switched on. Microscopic examination of electropolished flag electrodes showed the presence of ridges in the direction of gravity, which could be related to downward flow of a heavy viscous layer formed close to the electrode surface. The downward facing surface of an electropolished flag electrode showed the presence of pits, which could be related to local eddy currents caused near the surface by the dissolving products. Natural convection also affected the electropolishing 
behavior and rate for electrodes electropolished in different configuration by varying the diffusion layer thickness. 


\section{Chapter 6 Future Work}

The mechanism for niobium electropolishing was confirmed using electrochemical polarization and galvanostatic experiments. Transient time data obtained from potential transients showed a linear relationship with inverse square of current density and fluoride ion concentration square according to Sand's equation. However the linear curves did not pass through the origin, which should have been the case if the system followed a perfect semi - infinite linear diffusion model. This indicates the presence of other interfering processes in the electropolishing system. Natural convection seems one plausible reason and some evidence as to its presence was found in this work. However, more quantitative work needs to be done in order to determine the processes which could be affecting the niobium electropolishing system.

The effect of viscosity of the electrolyte solution on the mechanism can be investigated. The effect of natural convection might be less for the use of denser electrolyte. So, a different electrolyte recipe, possibly ionic liquids, can be used to study the effect.

The exact composition and nature of the viscous surface layer formed during $\mathrm{Nb}$ electropolishing is still unknown. In-situ experiments can be designed to find out the unknown nature of the layer.

The effect of variation of electropolishing current density on the surface finish can also be studied. The electron beam welded (EBW) region of the SRF cavities are especially 
prone to pitting as a result of electropolishing. Fundamental studies need to be done in order to ascertain the mechanism behind this defect formation. This is very essential for achieving the goals of pushing the accelerating gradients of the SRF cavities. 


\section{Bibliography}

H.Padamsee, J.Knobloch, and T.Hays, RF Superconductivity for Accelerators. 2 ed. 2008, Morlenbach: Wiley.

H.Padamsee, RF Superconductivity : Science, Technology, and Applications. 2009, Weinheim: Wiley-VCH.

V.Shemelin and H.Padamsee, Magnetic field enhancement at pits and bumps on the surface of superconducting cavities. 2008-07.

K.Saito, et al. Superiority of Electropolishing over Chemical Polishing on High Gradients. in 1997 Workshop on RF Superconductivity. 1997. Abano Terme (Padova), Italy.

Tian, H., et al., The mechanism of electropolishing of niobium in hydrofluoric-sulfuric acid electrolyte. Journal of the Electrochemical Society, 2008. 155(9): p. D563-D568.

Palmieri, V., et al. Besides the Standard Niobium Bath Chemical Polishing. in The 10th Workshop on RF Superconductivity. 2001. Tsukuba, Japan.

Eozenou, F., et al., Electropolishing of Niobium: best EP Parameters, in CAREReport-06-010-SRF. 2006. 
Cooley, L., et al., Impact of Forming, Welding, and Electropolishing on Pitting and the Surface Finish of SRF Cavity Niobium. 2010, Fermilab.

K.Saito, et al. Superiority of Electropolishing over Chemical Polishing on High Gradients. in 1997 Workshop on RF Superconductivity. 1997. Abano Terme (Padova), Italy.

T.Higuchi, et al. Investigation on Barrel Polishing for Superconducting Niobium Cavities. in 1995 Workshop on RF Superconductivity. 1995. Gif-sur-Yvette, France.

K.Saito. Development of Electropolishing Technology for Superconducting Cavities. in Particle Accelerator Conference. 2003. Portland, Oregon.

Fermilab. [cited; Available from: http://www.fnal.gov/pub/today/images10/10-050509D.jpg.

P.A.Jacquet, Electrolytic Method for obtaining Bright Copper Surfaces. Nature, 1935. 135(3426): p. 1076.

Chemical and Electrolytic Polishing, in ASM Handbook. 2004, ASM International. p. 281-293.

[cited; Available from: http://www.ableelectropolishing.com/0905able.pdf.

W.C.Elmore, Electrolytic Polishing. Journal of Applied Physics, 1939. 10: p. 724727.

Frankel, G., MSE 735 Kinetics II. 2010, Fontana Corrosion Center: Columbus 
Bockris, J.M. and A.K.N. Reddy, Modern Electrochemistry-Ionics. Vol. 1. 2002, New York: Kluwer Academic Publishers. 1232-1234.

Bard, A.J. and L.R. Faulkner, Electrochemical Methods: Fundamentals and Applications. 1980, New York: John Wiley \& Sons.

Landolt, D., Fundamental aspects of electropolishing. Electrochimica Acta, 1987. 32(1): p. 1-11.

Chandra, G.S.Frankel, and M.D.Sumption. Electropolishing of Niobium to Obtain Defect Free Surface. in SRF 2011. 2011. Chicago, USA.

Frankel, G., MSE 735 Kinetics I. 2010, Fontana Corrosion Center: Columbus. Landolt, D., Corrosion and surface chemistry of metals. 2007, Lausanne, Switzerland: EPFL Press.

A.J.Bard and L.R.Faulkner, Electrochemical Methods Fundamentals and Applications. 2 ed. 2001, New York, USA: John Wiley \& Sons.

Palmieri, V. Fundamentals of Electrochemistry - The Electrolytic polishing of Metals: Application to Copper and Niobium. in SRF Workshop 2003. 2003. Germany.

H.C.Kuo and D. Landolt, Rotating-disk electrode study of anodic dissolution of iron in concentrated chloride media. Electrochimica Acta, 1975. 20(5): p. 393-399.

D.J.Schiffrin, The influence of mass-transfer on the mechanism of electropolishing of nickel in aquesous sulfuric acid. Electrochimica Acta, 1982. 27(7): p. 837-845. 
Du, B. and I.I. Suni, Mechanistic studies of $\mathrm{Cu}$ in Electropolishing in Phosphoric Acid Electrolytes. Journal of the Electrochemical Society, 2004. 151(6): p. C375C378.

Diepers, H., et al., A New Method of Electropolishing Niobium. Physics Letters, 1971. 37A(2): p. 139-140.

Hoar, T.P., D.C. Mears, and G.P. Rothwell, The Relationships between anodic passivity, brightening and pitting. Corrosion Science, 1965. 5: p. 279-289.

$\begin{array}{llll}\text { Antoine. EP-Tutorial. } & \text { [cited; Available from: http://ilc- }\end{array}$ dms.fnal.gov/Members/tajima/References/Antoine_EP tutorial_01JUN2006.ppt/file view.

Landolt, D., P.F. Chauvy, and O. Zinger, Electrochemical micromachining, polishing and surface structuring of metals: fundamental aspects and new developments. Electrochimica Acta, 2003. 48(20-22): p. 3185-3201.

Matlosz, M., MODELING OF IMPEDANCE MECHANISMS IN ELECTROPOLISHING. Electrochimica Acta, 1995. 40(4): p. 393-401.

Glarum, S.H. and J.H. Marshall, The Anodic Dissolution of Copper into Phosphoric Acid. Journal of the Electrochemical Society, 1985. 132(12): p. 2878-2885.

Vidal, R. and A.C. West, Copper Electropolishing in Concentrated Phosphoric Acid I. Experimental Findings. Journal of the Electrochemical Society, 1995. 142(8): p. 2682-2689. 
A.C.West, et al., Electrohydrodynamic impedance study of anodically formed salt films on iron in chloride solutions. Journal of Electroanalytical Chemistry, 1992. 330(1-2): p. 693-706.

Mendez, J., et al., A Mechanistic Model for Copper Electropolishing in Phosphoric Acid. Journal of the Electrochemical Society, 2008. 155(1): p. D27-D34.

Strehblow, H.H. and J. Wenners, Investigation of the Processes on Iron and Nickel Electrodes at High Corrosion Current Densities in Solutions of High Chloride Content. Electrochimica Acta, 1976. 22: p. 421-427.

Padhi, D., et al., Planarization of Copper Thin Films by Electropolishing in Phosphoric Acid for ULSI Applications. Journal of the Electrochemical Society, 2003. 150(1): p. G10-G14.

Bockris, J.M., A.K.N. Reddy, and M. Aldeco, Modern Electrochemistry: Fundamental of Electrodics. 2nd ed. Vol. 2A. 2002, New York: Kluwer Academic Publishers.

Hoar, T.P. and D.C. Mears, Corrosion-Resistant Alloys in Chloride Solutions: Materials for Surgical Implants. Proceeding of the Royal Society A, 1966. 294: p. 486-510.

Frankel, G.S., Pitting Corrosion of Metals: A Review of the Critical Factors. Journal of the Electrochemical Society, 1998. 145(6): p. 2186-2198.

Beck, T.R. and R.C. Alkire, Occurence of Salt Films during Initiation and Growth of Corrosion Pits. Journal of the Electrochemical Society, 1979. 10(126): p. 1662-1666. 
U.F.Franck. Electrochemical Studies of Pitting Corrosion of Passive Metals. in 1st International Congress on Metallic Corrosion. 1961. London: Butterworths.

Frankel, G.S., et al., Metastable Pitting of Stainless Steel. Corrosion Science, 1987. 43(7): p. 429-436.

Vetter, K.J. and H.H. Strehblow. Pitting Corrosion in an Early Stage and its Theoretical Implications. in NACE 1974. Houston.

Chao, C.Y., L.F. Lin, and D.D. Macdonald, A POINT-DEFECT MODEL FOR ANODIC PASSIVE FILMS .1. FILM GROWTH-KINETICS. Journal of the Electrochemical Society, 1981. 128(6): p. 1187-1194.

Cooley, L., et al., Annealing to Mitigate Pitting in Electropolished Niobium coupons and SRF Cavities. 2011, Fermilab.

Datta, M. and D. Landolt, PIT AND FLOW STREAK FORMATION DURING HIGHRATE DISSOLUTION OF NICKEL. Journal of the Electrochemical Society, 1982. 129(9): p. 1889-1895.

Imboden, R. and R. Sibley, Anodic Polishing of Plain Carbon Steels. Transactions of the Electrochemical Society, 1942. 82: p. 227-239.

Madore, C. and D. Landolt, Electrochemical micromachining of controlled topographies on titanium for biological applications. Journal of Micromechanics and Microengineering, 1997. 7: p. 270-275. 
Palmieri, V., et al. Niobium Electropolishing by Ionic Liquids: What are the naked facts? in SRF 2009. 2009. Berlin, Germany.

Shieh, J.M., et al., Reduction of etch pits of electropolished Cu by additives. Journal of the Electrochemical Society, 2004. 151(7): p. C459-C462.

Tajiri, K., et al., Pit-free electropolishing of aluminum and its application for process chamber. Journal of Vacuum Science \& Technology a-Vacuum Surfaces and Films, 1998. 16(3): p. 1196-1200.

Van Gils, S., et al., Electropolishing of copper in H3PO4 - Ex situ and in situ optical characterization. Journal of the Electrochemical Society, 2007. 154(3): p. C175C180.

Cao, X. Analysis of Hydrogen Contamination of Superconducting Cavities. in The Fifth Workshop on RF Superconductivity. 1991. DESY, Hamburg, Germany.

Higuchi, T. and K.Saito, Hydrogen Absorption in Electropolishing of Niobium. American Institute of Physics Conference Proceedings, 2003. 671(1): p. 203-219.

Frankenthal, R., The Effect of Surface Preparation and of deformation on the Pitting and Anodic Dissolution of Fe-Cr Alloys. Corrosion Science, 1968. 8: p. 491-498.

http://lepp.cornell.edu/Research/AP/SRF/WebHome.html.

Zhao, X., Electropolishing of Niobium in Sulfuric Acid - Methanol Electrolytes: Development of Hydrofluoric Acid-Free Electrolytes, in Materials Science \& 
Engineering. 2009, Virginia Polytechnic Institute and State University: Blacksburg, Virginia. p. 16.

Madore, C., O. Piotrowski, and D. Landolt, Through-Mask Electrochemical Micromachining of Titanium. Journal of the Electrochemical Society, 1999. 146(7): p. $2526-2532$.

Frankel, G., MSE 735 Kinetics II. 2010, Fontana Corrosion Center: Columbus

Bard, A.J. and L.R. Faulkner, Electrochemical Methods: Fundamentals and Applications. 1980, New York: John Wiley \& Sons.

William D. Callister, J., Materials Science And Engineering An Introduction. 7 ed. 2007, New York: John Wiley \& Sons.

Tian, H., M.J. Kelley, and C.E. Reece, Evaluation of the fluoride ion diffusion coefficient in a sulfuric and hydrofluoric acid electrolyte during niobium electropolishing for superconducting radio frequency (SRF) accelerator cavities. Abstracts of Papers of the American Chemical Society, 2009. 238.

Eozenou, F., et al., Electropolishing of Niobium: best EP Parameters, in CAREReport-06-010-SRF. 2006.

Bockris, J.M. and A.K.N. Reddy, Modern Electrochemistry-Ionics. Vol. 1. 2002, New York: Kluwer Academic Publishers. 1232-1234.

Matlosz, M., Modeling of Impedance Mechanisms in Electropolishing. Electrochimica Acta, 1995. 40(4): p. 393-401. 
Landolt, D., Corrosion and Surface Chemistry of Metals. 1 ed. 2007, Lausanne, Switzerland: EPFL Press. 